\title{
Perylene Diimide-Embedded Double [8]Helicenes
}

Bo Liu ${ }^{\dagger, \S}$ Marcus Böckmann, ${ }^{\#}$ Wei Jiang, ${ }^{*, \dagger, \S}$ Nikos L. Doltsinis, ${ }^{\#}$ and Zhaohui Wang*,†, $*$ \&

${ }^{\dagger}$ Beijing National Laboratory for Molecular Sciences, CAS Key Laboratory of Organic Solids, CAS Research/Education Center for Excellence in Molecular Sciences, Institute of Chemistry, Chinese Academy of Sciences, Beijing 100190, China

$\$$ Key Laboratory of Organic Optoelectronics and Molecular Engineering, Department of Chemistry, Tsinghua University, Beijing 100084, China

\# Institute for Solid State Theory and Center for Multiscale Theory \& Computation, University of Muenster, Wilhelm-Klemm-Strasse 10, 48149 Muenster, Germany

$\S$ University of Chinese Academy of Sciences, Beijing 100049, China

\section{Table of Contents}

1. Materials and Measurements...............................................S2

2. Synthetic Details........................................................S3

3. Optical, Electrochemical, and Thermal Properties...........................S9

4. Chiral HPLC Chromatogram..........................................S14

5. CD and CPL Spectra..................................................S16

6. X-ray Crystal Structure....................................................S18

7. Theoretical Calculations................................................... S23

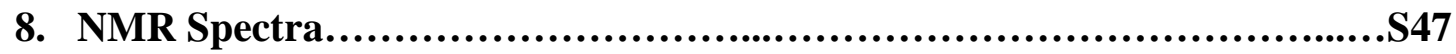

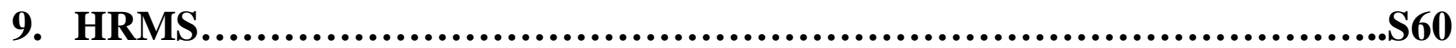

10. References................................................................65 


\section{Materials and Measurements}

Unless otherwise mentioned, all commercials were used as received without further purification.

${ }^{1} \mathrm{H}$ NMR (500 MHz) and ${ }^{13} \mathrm{C}$ NMR (125 MHz) spectra were recorded on a Bruker ADVANCE 500 NMR Spectrometer using $\mathrm{CDCl}_{3}$ and $\mathrm{C}_{2} \mathrm{D}_{2} \mathrm{Cl}_{4}$ as deuterated solvents. Chemical shifts $(\delta)$ are reported in ppm. The signals have been designated as follows: $\mathrm{s}$ (singlet), d (doublet), t (triplet), dd (doublet doublet), m (multiplet), coupling constant $J(\mathrm{~Hz})$. High resolution mass spectra (HRMS) were determined on IonSpec 4.7 Tesla Fourier Transform Mass Spectrometer.

UV-Vis spectra were measured with Hitachi (Model U-3010) UV-Vis spectrophotometer in $\mathrm{CHCl}_{3}$ in a 1-cm quartz cell. Fluorescence measurements were acquired on a Jasco FP-660 Spectrofluorometer in $\mathrm{CHCl}_{3}$ at room temperature. Fluorescence quantum yields and lifetime were determined by FLS980 fluorimeter in $\mathrm{CHCl}_{3}$ at room temperature. Cyclic Voltammetry (CV) measurements were carried out with a CHI620E electrochemical workstation using glassy carbon discs as the working electrode, $\mathrm{Pt}$ wire as the counter electrode, $\mathrm{Ag} / \mathrm{AgCl}$ electrode as the reference electrode at a scanning rate of $100 \mathrm{mV} / \mathrm{s}$. $0.1 \mathrm{M}$ tetrabutylammonium hexafluorophosphate $\left(\mathrm{Bu}_{4} \mathrm{NPF}_{6}\right)$ dissolved in $\mathrm{CH}_{2} \mathrm{Cl}_{2}$ was employed as the supporting electrolyte. Thermogravimetric analysis (TGA) measurements were performed on a TGA 8000 instrument, heating from $30^{\circ} \mathrm{C}$ to $600{ }^{\circ} \mathrm{C}$, at a heating rate of $10{ }^{\circ} \mathrm{C} / \mathrm{min}$ under a dry nitrogen flow.

CD spectra were collected on JASCO J-810 circular dichroism spectrometer. CPL spectra were collected on JASCO CPL-300 circularly polarized luminescence spectrometer.

Single crystals data collections were performed on a Bruker SMART CCD area detector with graphite monochromated $\mathrm{CuK} \alpha$ radiation $(\lambda=1.54184)$. All calculations were performed using the SHELXL and the crystal structure crystallographic software package. 


\section{Synthetic Details}

1-Bromo-perylene-3,4,9,10-tetracarboxylic diimides ${ }^{[1]}$

1,6-dibromoperylene-3,4,9,10-tetracarboxylic

diimides, ${ }^{[2]}$

1,7-dibromoperylene-3,4,9,10-tetracarboxylic

diimides $^{[2]}$

and

4,4,5,5-tetramethyl-2-([6]helicene-2-yl)-1,3,2-dioxaborolane ${ }^{[3]}$ were prepared according to the literature.

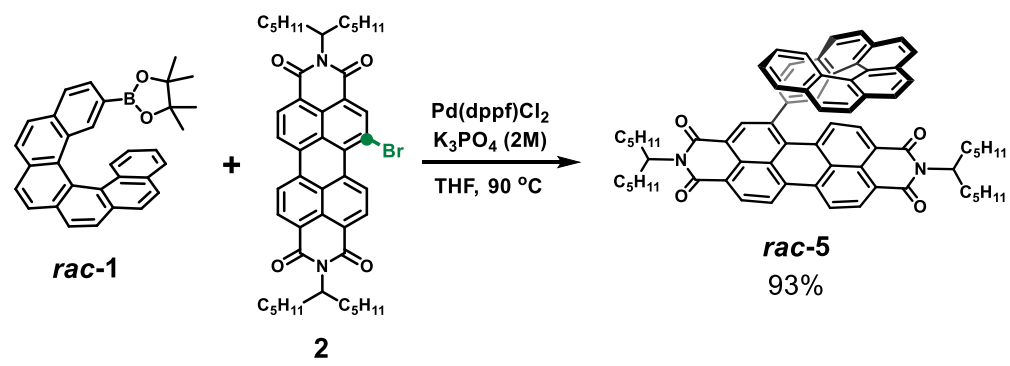

Scheme S1. Synthetic Routes to $r a c-5$

A Schlenk flask was charged with 1-bromoperylene-3,4,9,10-tetracarboxylic diimides (2, $100 \mathrm{mg}, 0.13 \mathrm{mmol})$ and $\mathbf{r a c}$-1 (4,4,5,5-tetramethyl-2-([6]helicene-2-yl)-1,3,2-dioxaborolane) (70 mg, $0.15 \mathrm{mmol})$, THF ( $2 \mathrm{~mL}$ ) and $\mathrm{K}_{3} \mathrm{PO}_{4}$ aqueous solution $(1 \mathrm{~mL}, 2 \mathrm{M})$. The mixture was degassed with argon for $20 \mathrm{~min} . \mathrm{Pd}(\mathrm{dppf}) \mathrm{Cl}_{2}(10 \mathrm{mg}, 0.013 \mathrm{mmol})$ was added under an argon atmosphere. The mixture was heated at $90{ }^{\circ} \mathrm{C}$ for $24 \mathrm{~h}$ and then cooled down to room temperature. The mixture was diluted with water $(100 \mathrm{~mL})$, then extracted with $\mathrm{CH}_{2} \mathrm{Cl}_{2}$ (200 $\mathrm{mL})$, washed with brine $(200 \mathrm{~mL})$, dried over anhydrous $\mathrm{Na}_{2} \mathrm{SO}_{4}$ and concentrated in vacuo. The crude product was purified by column chromatography on silica gel, eluted with petroleum ether $/ \mathrm{CH}_{2} \mathrm{Cl}_{2}$ (4: 1 to $3: 1 \mathrm{v} / \mathrm{v}$ ) to afford compound $\mathbf{r a c}-\mathbf{5}$ as dark red solids (124 $\mathrm{mg}$, 93\%). ${ }^{1} \mathrm{H}$ NMR (500 MHz, 373.2 K, $\left.\mathrm{C}_{2} \mathrm{D}_{2} \mathrm{Cl}_{4}\right): \delta 8.67-8.58(\mathrm{~m}, 4 \mathrm{H}), 8.14-7.43(\mathrm{~m}, 15 \mathrm{H}), 7.18$ (s, 2H), $6.94(\mathrm{~s}, 1 \mathrm{H}), 5.31(\mathrm{~s}, 1 \mathrm{H}), 5.09(\mathrm{~s}, 1 \mathrm{H}), 2.43(\mathrm{~s}, 2 \mathrm{H}), 2.17(\mathrm{~s}, 2 \mathrm{H}), 2.02(\mathrm{~s}, 2 \mathrm{H}), 1.88(\mathrm{~s}$, 2H), $1.32(\mathrm{~s}, 24 \mathrm{H}), 0.97-0.88(\mathrm{~m}, 12 \mathrm{H}) ;{ }^{13} \mathrm{C} \mathrm{NMR}\left(125 \mathrm{MHz}, 373.2 \mathrm{~K}, \mathrm{C}_{2} \mathrm{D}_{2} \mathrm{Cl}_{4}\right): \delta 163.9$, $163.6,163.3,141.4,135.9,134.4,134.2,133.2,132.0,131.6,131.4,131.3,130.6,130.3$, $129.9,129.5,128.8,128.2,127.9,127.6,127.3,127.2,127.1,126.6,125.9,124.7,123.9$, 123.4, 122.9, 122.1, 54.6, 32.4, 32.3, 31.6, 31.5, 26.4, 22.3, 22.2, 13.7, 13.6; HRMS (MALDI, 100\%): $\mathrm{m} / \mathrm{z}$ calcd (\%) for $\mathrm{C}_{72} \mathrm{H}_{68} \mathrm{~N}_{2} \mathrm{O}_{4}: 1024.5179$, found: 1024.5179. 


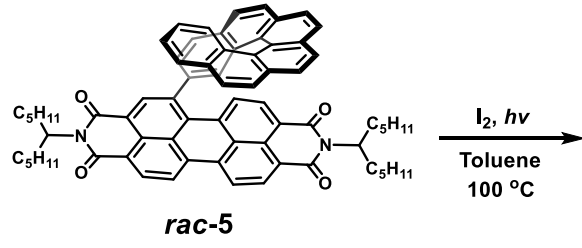

rac-5

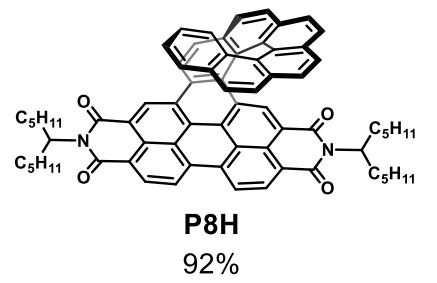

$92 \%$

Scheme S2. Synthetic Routes to P8H

To a standard photocyclization glassware were added $300 \mathrm{mg}$ rac $\mathbf{- 5}(10 \times 30 \mathrm{mg}), 500 \mathrm{~mL}$ toluene $(10 \times 50 \mathrm{~mL})$ and $5 \mathrm{mg} \mathrm{I}$. The mixture was irradiated by $40 \mathrm{~W}$ LED lamp at $100{ }^{\circ} \mathrm{C}$ for $2 \mathrm{~h}$. After the reaction, the mixture was washed with saturated sodium sulfite solution and concentrated under reduced pressure. The crude product was purified by column chromatography on silica gel, eluted with petroleum ether/ $\mathrm{CH}_{2} \mathrm{Cl}_{2}(2: 1, \mathrm{v} / \mathrm{v})$ to afford compound P8H (275 mg, 92\%) as a red solid. ${ }^{1} \mathrm{H}$ NMR (500 MHz, $328.2 \mathrm{~K}, \mathrm{CDCl}_{3}$ ): $\delta 9.71$ (s, 1H), 9.18 (d, $J=8.2 \mathrm{~Hz}, 1 \mathrm{H}), 9.10(\mathrm{~d}, J=8.1 \mathrm{~Hz}, 1 \mathrm{H}), 9.03$ (d, $J=8.1 \mathrm{~Hz}, 1 \mathrm{H}), 8.92(\mathrm{~d}, J$ $=8.1 \mathrm{~Hz}, 1 \mathrm{H}), 8.64(\mathrm{~d}, J=8.5 \mathrm{~Hz}, 1 \mathrm{H}), 8.32(\mathrm{~s}, 1 \mathrm{H}), 8.27(\mathrm{t}, J=8.2 \mathrm{~Hz}, 2 \mathrm{H}), 8.20(\mathrm{t}, J=7.9$ $\mathrm{Hz}, 2 \mathrm{H}), 8.10(\mathrm{~d}, J=8.1 \mathrm{~Hz}, 1 \mathrm{H}), 7.67(\mathrm{~d}, J=8.0 \mathrm{~Hz}, 1 \mathrm{H}), 7.07$ (d, $J=8.1 \mathrm{~Hz}, 1 \mathrm{H}), 6.87$ (d, $J$ $=8.4 \mathrm{~Hz}, 1 \mathrm{H}), 6.48(\mathrm{t}, J=7.4 \mathrm{~Hz}, 1 \mathrm{H}), 6.28(\mathrm{t}, J=7.7 \mathrm{~Hz}, 1 \mathrm{H}), 6.11(\mathrm{~d}, J=8.4 \mathrm{~Hz}, 1 \mathrm{H}), 5.89$ (d, $J=8.2 \mathrm{~Hz}, 2 \mathrm{H}), 5.40-5.35(\mathrm{~m}, 1 \mathrm{H}), 5.19(\mathrm{~s}, 1 \mathrm{H}), 2.41(\mathrm{~d}, J=9.4 \mathrm{~Hz}, 3 \mathrm{H}), 2.04(\mathrm{~s}, 3 \mathrm{H})$, $1.63(\mathrm{~s}, 5 \mathrm{H}), 1.48-1.35(\mathrm{~m}, 16 \mathrm{H}), 1.13(\mathrm{~s}, 7 \mathrm{H}), 0.89(\mathrm{t}, J=6.1 \mathrm{~Hz}, 7 \mathrm{H}), 0.72(\mathrm{~s}, 3 \mathrm{H}) ;{ }^{13} \mathrm{C}$ NMR (125 MHz, $\left.328.2 \mathrm{~K}, \mathrm{CDCl}_{3}\right): \delta$ 165.1, 165.0, 164.6, 133.8, 133.4, 132.2, 132.0, 131.6, 130.5, 129.2, 129.0,128.6, 128.3, 127.7, 127.3, 127.2, 126.9, 126.8, 126.4, 125.9, 125.6, 125.1, $124.9,124.7,124.3,124.2,124.1,124.0,122.9,122.8,122.5,121.3,55.3,54.9,32.9,32.8$, 32.1, 27.1, 27.0, 22.9, 22.8, 14.2. HRMS (MALDI, 100\%): $\mathrm{m} / \mathrm{z}$ calcd (\%) for $\mathrm{C}_{72} \mathrm{H}_{66} \mathrm{~N}_{2} \mathrm{O}_{4}$ : 1022.5023, found: 1022.5021 .

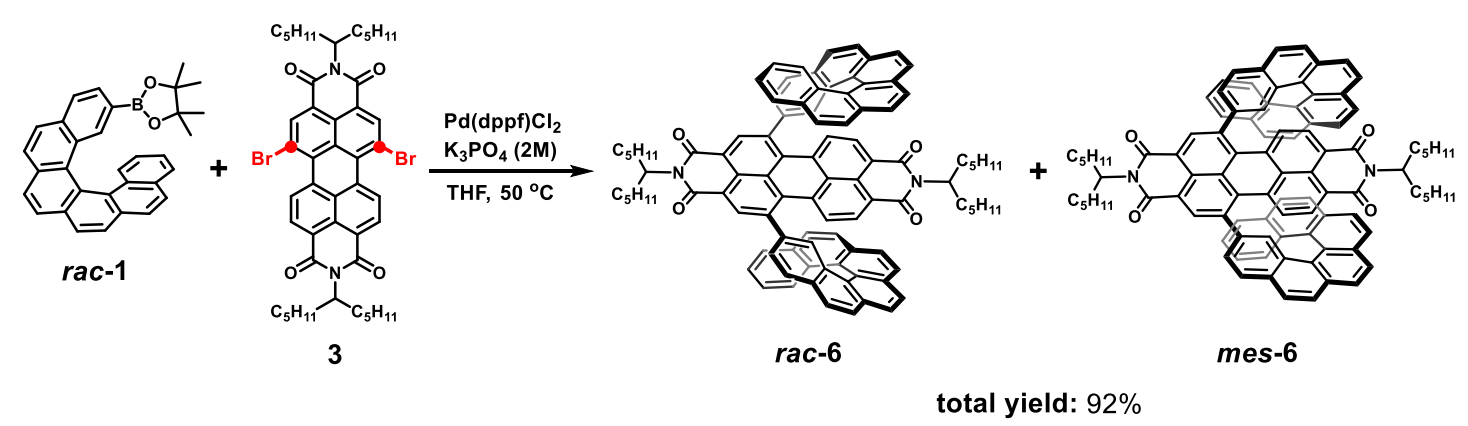

Scheme S3. Synthetic Routes to rac-6 and mes-6 
A Schlenk flask was charged with 1,6-dibromoperylene-3,4,9,10-tetracarboxylic diimides (3, $240 \mathrm{mg}, 0.28 \mathrm{mmol})$, rac $-\mathbf{1}(280 \mathrm{mg}, 0.62 \mathrm{mmol})$, THF $(6 \mathrm{~mL})$ and $\mathrm{K}_{3} \mathrm{PO}_{4}$ aqueous solution $(3 \mathrm{~mL}, 2 \mathrm{M})$. The mixture was degassed with argon for $20 \mathrm{~min}, \mathrm{Pd}(\mathrm{dppf}) \mathrm{Cl}_{2}(20 \mathrm{mg}$, $0.028 \mathrm{mmol}$ ) was added under an argon atmosphere. The mixture was heated at $50{ }^{\circ} \mathrm{C}$ for $6 \mathrm{~h}$ and then cooled down to room temperature. The mixture was diluted with water $(100 \mathrm{~mL})$, then extracted with $\mathrm{CH}_{2} \mathrm{Cl}_{2}(200 \mathrm{~mL})$, washed with brine $(200 \mathrm{~mL})$, dried over anhydrous $\mathrm{Na}_{2} \mathrm{SO}_{4}$ and concentrated in vacuo, and purified by column chromatography on silica gel, eluted with petroleum ether/ $\mathrm{CH}_{2} \mathrm{Cl}_{2}(3: 1, \mathrm{v} / \mathrm{v})$ to afford the title compound as dark red solids containing rac-6 and mes-6 as mixture (348 mg, 92\%). ${ }^{1} \mathrm{H}$ NMR (500 MHz, $373.2 \mathrm{~K}$, $\left.\mathrm{C}_{2} \mathrm{D}_{2} \mathrm{Cl}_{4}\right): \delta 8.10-7.84(\mathrm{~m}, 26 \mathrm{H}), 7.37(\mathrm{~s}, 4 \mathrm{H}), 6.93(\mathrm{~s}, 6 \mathrm{H}), 5.25(\mathrm{~s}, 1 \mathrm{H}), 5.05(\mathrm{~s}, 1 \mathrm{H}), 2.40(\mathrm{~s}$, $2 \mathrm{H}), 2.07-1.88(\mathrm{~d}, 6 \mathrm{H}), 1.31(\mathrm{~s}, 24 \mathrm{H}), 1.03-0.88(\mathrm{~d}, 12 \mathrm{H}) ;{ }^{13} \mathrm{C}$ NMR $(125 \mathrm{MHz}, 373.2 \mathrm{~K}$, $\left.\mathrm{C}_{2} \mathrm{D}_{2} \mathrm{Cl}_{4}\right): \delta 163.7,163.4,163.0,141.4,134.8,133.6,133.2,131.9,131.8,131.5,131.3,129.5$, $129.0,127.9,127.4,127.2,126.6,126.3,125.7,124.7,124.6,123.9,122.2,121.9,54.4,32.5$, 32.3, 31.6, 31.5, 26.5, 22.4, 22.2, 13.8, 13.6; HRMS (MALDI, 100\%): $\mathrm{m} / \mathrm{z}$ calcd (\%) for $\mathrm{C}_{98} \mathrm{H}_{82} \mathrm{~N}_{2} \mathrm{O}_{4}:$ 1351.6308, found: 1351.6324 .

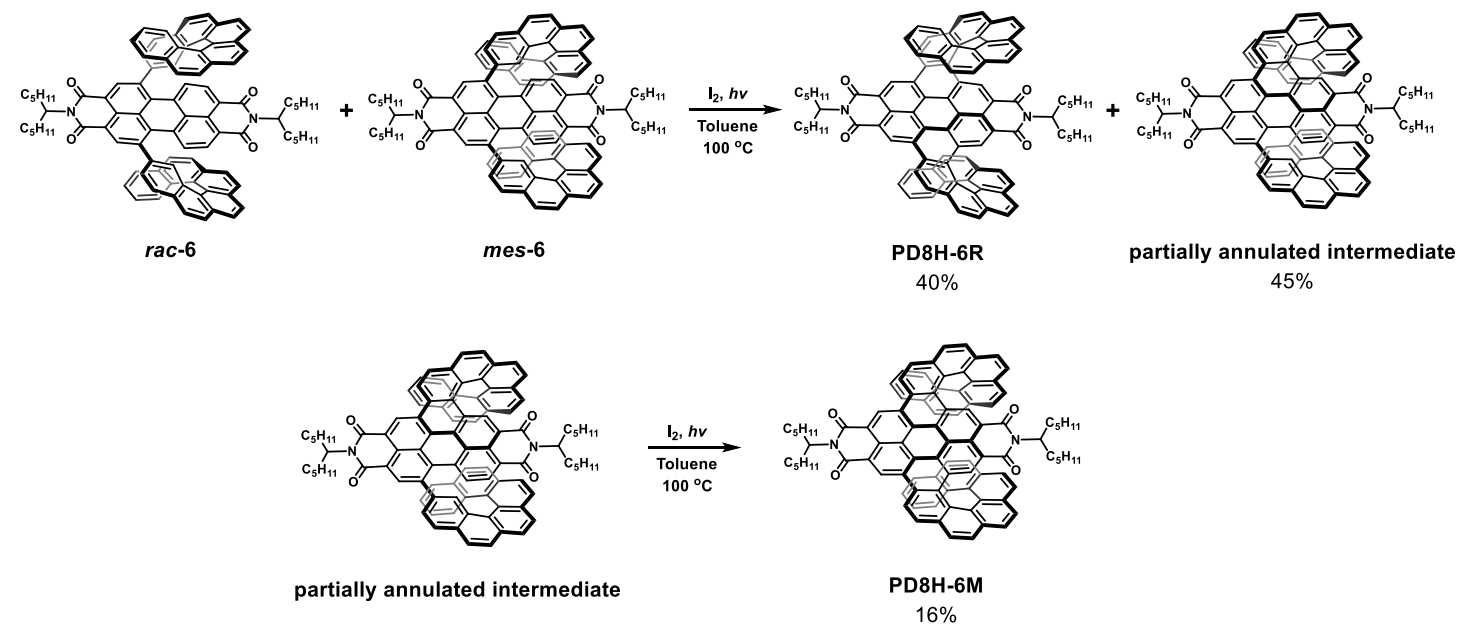

Scheme S4. Synthetic Routes to PD8H-6R and PD8H-6M

To a standard photocyclization glassware were added $300 \mathrm{mg}$ mixture of rac-6 and mes-6 $(10 \times 30 \mathrm{mg}), 500 \mathrm{~mL}$ toluene $(10 \times 50 \mathrm{~mL})$ and $5 \mathrm{mg} \mathrm{I}_{2}$. The mixture was irradiated by $40 \mathrm{~W}$ LED lamp at $100{ }^{\circ} \mathrm{C}$ for 5 days. After the reaction, the mixture was washed with saturated sodium sulfite solution and concentrated under reduced pressure. The crude product was purified by column chromatography on silica gel, eluted with petroleum ether/ $\mathrm{CH}_{2} \mathrm{Cl}_{2}(5: 1$, 
v/v) to afford the title compound PD8H-6R (120 mg, 40\%, this yield is determined by the mixture) as a red solid and the partially annulated intermediate $(135 \mathrm{mg}, 45 \%)$ as a red solid. HRMS (MALDI, 100\%): $\mathrm{m} / z$ calcd (\%) for $\mathrm{C}_{98} \mathrm{H}_{80} \mathrm{~N}_{2} \mathrm{O}_{4}: 1349.6152$, found: 1349.6167.

PD8H-6R: ${ }^{1} \mathrm{H}$ NMR $\left(500 \mathrm{MHz}, 373.2 \mathrm{~K}, \mathrm{C}_{2} \mathrm{D}_{2} \mathrm{Cl}_{4}\right): \delta 10.20(\mathrm{~s}, 2 \mathrm{H}), 9.05(\mathrm{~d}, J=8.5 \mathrm{~Hz}, 2 \mathrm{H})$, $8.79(\mathrm{~s}, 2 \mathrm{H}), 8.48(\mathrm{~d}, J=8.5 \mathrm{~Hz}, 2 \mathrm{H}), 8.38(\mathrm{~d}, J=7.3 \mathrm{~Hz}, 4 \mathrm{H}), 8.28(\mathrm{~d}, J=8.2 \mathrm{~Hz}, 2 \mathrm{H}), 8.14$ $(\mathrm{d}, J=8.1 \mathrm{~Hz}, 2 \mathrm{H}), 7.59(\mathrm{~d}, J=8.0 \mathrm{~Hz}, 2 \mathrm{H}), 6.93(\mathrm{~d}, J=8.3 \mathrm{~Hz}, 2 \mathrm{H}), 6.74(\mathrm{~d}, J=8.0 \mathrm{~Hz}, 2 \mathrm{H})$, $6.47(\mathrm{t}, J=7.3 \mathrm{~Hz}, 2 \mathrm{H}), 6.38(\mathrm{t}, J=7.6 \mathrm{~Hz}, 2 \mathrm{H}), 5.67-5.61(\mathrm{~m}, 1 \mathrm{H}), 5.48(\mathrm{~d}, J=7.8 \mathrm{~Hz}, 2 \mathrm{H})$, $5.38(\mathrm{~d}, J=8.4 \mathrm{~Hz}, 2 \mathrm{H}), 5.26-5.21(\mathrm{~m}, 1 \mathrm{H}), 4.91(\mathrm{~d}, J=8.4 \mathrm{~Hz}, 2 \mathrm{H}), 2.73-2.62(\mathrm{~m}, 2 \mathrm{H})$, 2.42-2.32 (m, 2H), 2.28-2.21 (m, 2H), 2.17-2.06 (m, 2H), 1.73-1.53 (m, 20H), 1.50-1.46 (m, 4H), 1.09-1.05 (q, 6H), $0.98(\mathrm{q}, J=6.9 \mathrm{~Hz}, 6 \mathrm{H}) ;{ }^{13} \mathrm{C} \mathrm{NMR}\left(125 \mathrm{MHz}, 373.2 \mathrm{~K}, \mathrm{C}_{2} \mathrm{D}_{2} \mathrm{Cl}_{4}\right): \delta$ $165.5,164.2,133.1,131.9,131.6,130.6,129.5,128.6,128.4,128.3,128.0,127.4,127.2$, $127.1,126.6,126.5,126.4,126.2,125.6,125.5,124.7,124.3,124.1,124.0,123.8,123.6$, $123.5,123.4,123.2,122.2,121.6,121.4,121.2,120.8,120.4,55.1,54.5,33.0,32.7,32.6,32.1$, 31.6, 27.1, 27.0, 26.7, 26.6, 22.6, 22.5, 22.4, 13.9, 13.7; HRMS (MALDI, 100\%): $\mathrm{m} / \mathrm{z}$ calcd (\%) for $\mathrm{C}_{98} \mathrm{H}_{78} \mathrm{~N}_{2} \mathrm{O}_{4}: 1347.5995$, found: $1347.6016 .[\alpha]_{D}{ }^{27}=+10804^{\circ}$.

The partially annulated intermediate $(135 \mathrm{mg})$ was then irradiated by $40 \mathrm{~W}$ LED lamp at $100{ }^{\circ} \mathrm{C}$ for one month to afford the title compound PD8H-6M as an orange solid (22 mg, $16 \%$, this yield is determined by partially annulated intermediate). ${ }^{1} \mathrm{H}$ NMR $(500 \mathrm{MHz}, 373.2 \mathrm{~K}$, $\left.\mathrm{C}_{2} \mathrm{D}_{2} \mathrm{Cl}_{4}\right): \delta 10.20(\mathrm{~s}, 2 \mathrm{H}), 8.92(\mathrm{~d}, J=8.5 \mathrm{~Hz}, 2 \mathrm{H}), 8.77(\mathrm{~s}, 2 \mathrm{H}), 8.39-8.35(\mathrm{~m}, 6 \mathrm{H}), 8.30(\mathrm{t}, J$ $=10.0 \mathrm{~Hz}, 4 \mathrm{H}), 8.02(\mathrm{~d}, J=8.1 \mathrm{~Hz}, 2 \mathrm{H}), 7.54(\mathrm{~d}, J=8.4 \mathrm{~Hz}, 2 \mathrm{H}), 7.18(\mathrm{~d}, J=8.4 \mathrm{~Hz}, 2 \mathrm{H})$, 6.99 (t, $J=7.4 \mathrm{~Hz}, 2 \mathrm{H}), 6.91(\mathrm{~d}, J=8.3 \mathrm{~Hz}, 2 \mathrm{H}), 6.70(\mathrm{t}, J=7.7 \mathrm{~Hz}, 2 \mathrm{H}), 6.59$ (d, $J=8.0 \mathrm{~Hz}$, 2H), $6.38(\mathrm{~d}, J=8.3 \mathrm{~Hz}, 2 \mathrm{H}), 5.55(\mathrm{t}, J=6.0 \mathrm{~Hz}, 1 \mathrm{H}), 5.14(\mathrm{~s}, 1 \mathrm{H}), 2.55(\mathrm{~s}, 2 \mathrm{H}), 2.26(\mathrm{~s}, 2 \mathrm{H})$, 1.90 (s, 2H), 1.65-1.35 (m, 26H), 0.99 (s, 12H); $\left.{ }^{13} \mathrm{C} \mathrm{NMR} \mathrm{(125} \mathrm{MHz,} 373.2 \mathrm{~K}, \mathrm{C}_{2} \mathrm{D}_{2} \mathrm{Cl}_{4}\right): \delta$ $165.4,133.3,131.9,131.5,131.1,130.1,128.5,128.3,128.1,127.9,127.8,127.5,126.9$, $126.7,126.5,125.9,125.7,125.4,125.2,124.7,124.6,123.9,123.4,123.2,122.9,122.7$, $122.1,121.5,121.2,119.6,55.1,54.2,32.6,32.4,31.9,31.6,26.7,22.3,13.7$; HRMS (MALDI, 100\%): $\mathrm{m} / z$ calcd (\%) for $\mathrm{C}_{98} \mathrm{H}_{78} \mathrm{~N}_{2} \mathrm{O}_{4}: 1347.5995$, found: 1347.6006 . 


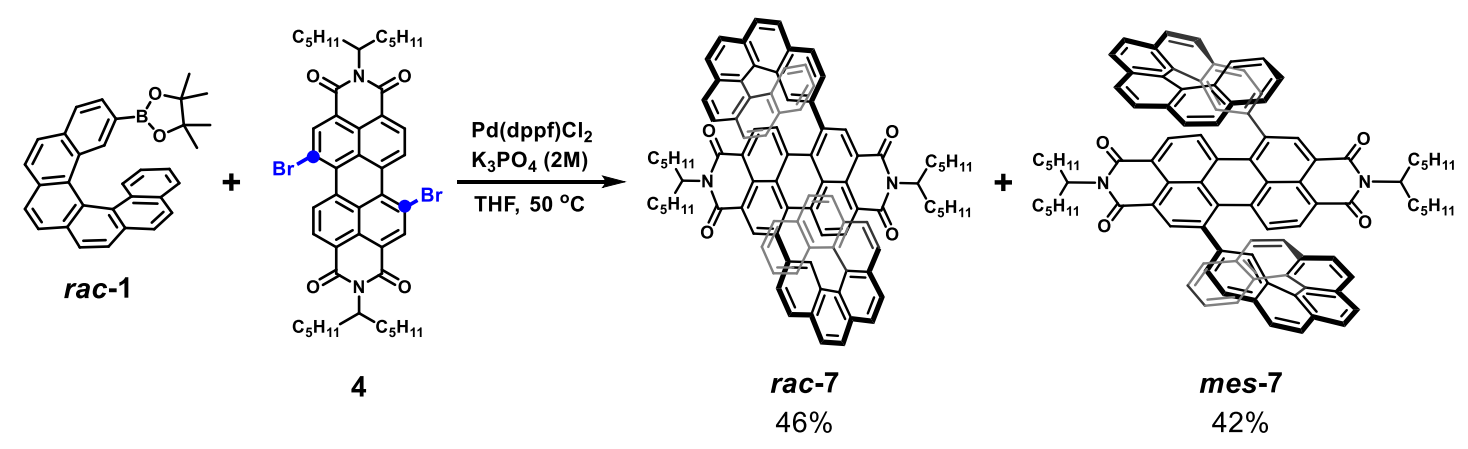

Scheme S5. Synthetic Routes to rac-7 and mes-7

A Schlenk flask was charged with 1,7-dibromoperylene-3,4,9,10-tetracarboxylic diimides (4, $240 \mathrm{mg}, 0.28 \mathrm{mmol})$, $r a c-1(280 \mathrm{mg}, 0.62 \mathrm{mmol})$, THF $(6 \mathrm{~mL})$ and $\mathrm{K}_{3} \mathrm{PO}_{4}$ aqueous solution $(3 \mathrm{~mL}, 2 \mathrm{M})$. The mixture was degassed with argon for $20 \mathrm{~min}, \mathrm{Pd}(\mathrm{dppf}) \mathrm{Cl}_{2}(20 \mathrm{mg}$, $0.028 \mathrm{mmol}$ ) was added under an argon atmosphere. The mixture was heated at $50{ }^{\circ} \mathrm{C}$ for $6 \mathrm{~h}$ and then cooled down to room temperature. The mixture was diluted with water (100 mL), then extracted with $\mathrm{CH}_{2} \mathrm{Cl}_{2}(200 \mathrm{~mL})$, washed with brine $(200 \mathrm{~mL})$, dried over anhydrous $\mathrm{Na}_{2} \mathrm{SO}_{4}$ and concentrated in vacuo, and purified by column chromatography on silica gel, eluted with petroleum ether/ $\mathrm{CH}_{2} \mathrm{Cl}_{2}(5: 1 \mathrm{v} / \mathrm{v})$ to afford $\mathbf{r a c}-7$ (174 mg, 46\%) and mes-7 (160 mg, 42\%) as dark red solids.

rac-7: ${ }^{1} \mathrm{H}$ NMR $\left(500 \mathrm{MHz}, 373.2 \mathrm{~K}, \mathrm{C}_{2} \mathrm{D}_{2} \mathrm{Cl}_{4}\right): \delta 8.12-7.86(\mathrm{~m}, 27 \mathrm{H}), 7.35(\mathrm{t}, J=7.5 \mathrm{~Hz}, 3 \mathrm{H})$, $7.12(\mathrm{~s}, 3 \mathrm{H}), 6.94(\mathrm{~s}, 3 \mathrm{H}), 5.17(\mathrm{~s}, 2 \mathrm{H}), 2.27(\mathrm{~s}, 4 \mathrm{H}), 1.95(\mathrm{~s}, 4 \mathrm{H}), 1.41(\mathrm{~s}, 24 \mathrm{H}), 0.96(\mathrm{~s}, 12 \mathrm{H})$; ${ }^{13} \mathrm{C}$ NMR (125 MHz, $\left.373.2 \mathrm{~K}, \mathrm{C}_{2} \mathrm{D}_{2} \mathrm{Cl}_{4}\right): \delta 163.8,163.3,140.5,138.9,134.9,134.1,133.2$, $132.1,131.8,131.5,131.4,129.6,128.9,128.6,127.9,127.7,127.3,127.2,127.1,126.6$, 125.6, 124.6, 123.9, 122.4, 121.8, 54.4, 32.4, 31.5, 26.4, 22.3, 13.7; HRMS (MALDI, 100\%): $m / z$ calcd (\%) for $\mathrm{C}_{98} \mathrm{H}_{82} \mathrm{~N}_{2} \mathrm{O}_{4}: 1351.6308$, found: 1351.6311 .

mes-7: ${ }^{1} \mathrm{H}$ NMR $\left(500 \mathrm{MHz}, 373.2 \mathrm{~K}, \mathrm{C}_{2} \mathrm{D}_{2} \mathrm{Cl}_{4}\right): \delta$ 8.12-7.56 (m, 27H), $7.34(\mathrm{~s}, 3 \mathrm{H}), 7.13(\mathrm{~s}$, 3H), 6.98 (s, 3H), $5.17(\mathrm{~s}, 2 \mathrm{H}), 2.28(\mathrm{~s}, 4 \mathrm{H}), 1.95(\mathrm{~s}, 4 \mathrm{H}), 1.41(\mathrm{~s}, 24 \mathrm{H}), 0.96(\mathrm{~s}, 12 \mathrm{H}) ;{ }^{13} \mathrm{C}$ NMR (125 MHz, $\left.373.2 \mathrm{~K}, \mathrm{C}_{2} \mathrm{D}_{2} \mathrm{Cl}_{4}\right): \delta 163.8,163.3,140.5,138.9,134.9,134.0,133.1,132.0$, $131.8,131.5,129.6,129.0,128.9,128.5,127.9,127.7,127.3,127.2,127.1,126.6,125.7$, 124.6, 123.9, 121.8, 54.3, 32.4, 31.5, 26.4, 22.3, 13.7; HRMS (MALDI, 100\%): $\mathrm{m} / \mathrm{z}$ calcd (\%) for $\mathrm{C}_{98} \mathrm{H}_{82} \mathrm{~N}_{2} \mathrm{O}_{4}:$ : 1351.6308, found: 1351.6313 . 

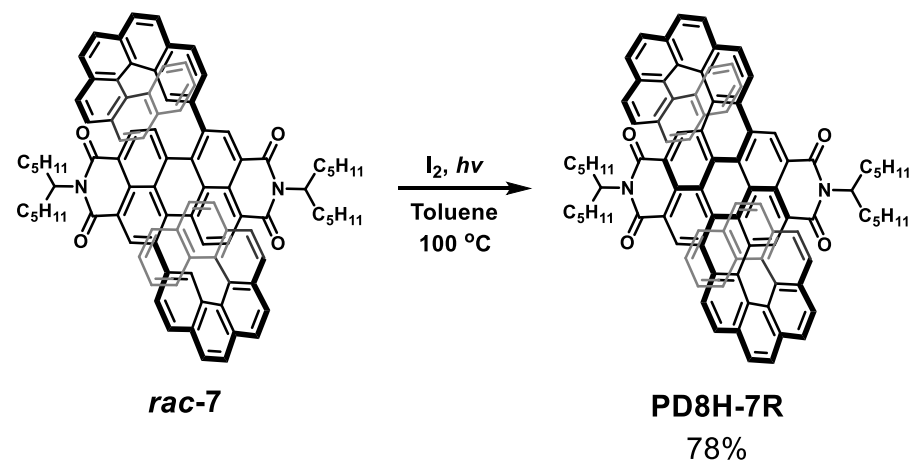

Scheme S6. Synthetic Routes to PD8H-7R

To a standard photocyclization glassware were added $300 \mathrm{mg}$ compound $\mathbf{r a c}-\mathbf{7}(10 \times 30$ $\mathrm{mg}), 500 \mathrm{~mL}$ toluene $(10 \times 50 \mathrm{~mL})$ and $5 \mathrm{mg} \mathrm{I}_{2}$. The mixture was irradiated by $40 \mathrm{~W}$ LED lamp at $100{ }^{\circ} \mathrm{C}$ for two weeks. After the reaction, the mixture was washed with saturated sodium sulfite solution and concentrated under reduced pressure. The crude product was purified by column chromatography on silica gel, eluted with petroleum ether/ $\mathrm{CH}_{2} \mathrm{Cl}_{2}(5: 1$, v/v) to afford the title compound PD8H-7R (234 mg, 78\%) as an orange solid. ${ }^{1} \mathrm{H}$ NMR (500 MHz, $\left.373.2 \mathrm{~K}, \mathrm{C}_{2} \mathrm{D}_{2} \mathrm{Cl}_{4}\right): \delta 10.18(\mathrm{~s}, 2 \mathrm{H}), 9.07(\mathrm{~d}, J=8.6 \mathrm{~Hz}, 2 \mathrm{H}), 8.93(\mathrm{~s}, 2 \mathrm{H}), 8.43-8.33(\mathrm{~m}$, $10 \mathrm{H}), 7.98(\mathrm{~d}, J=8.2 \mathrm{~Hz}, 2 \mathrm{H}), 7.46(\mathrm{~d}, J=8.2 \mathrm{~Hz}, 2 \mathrm{H}), 7.25(\mathrm{~d}, J=8.4 \mathrm{~Hz}, 2 \mathrm{H}), 6.98(\mathrm{t}, J=$ $8.4 \mathrm{~Hz}, 2 \mathrm{H}), 6.67-6.59(\mathrm{~m}, 6 \mathrm{H}), 6.50(\mathrm{~d}, J=8.4 \mathrm{~Hz}, 2 \mathrm{H}), 5.36(\mathrm{~s}, 2 \mathrm{H}), 2.45(\mathrm{~s}, 4 \mathrm{H}), 2.10(\mathrm{~s}$, 4H), 1.56 (s, 24H), $1.00(\mathrm{~s}, 12 \mathrm{H}) .{ }^{13} \mathrm{C}$ NMR (125 MHz, $\left.373.2 \mathrm{~K}, \mathrm{C}_{2} \mathrm{D}_{2} \mathrm{Cl}_{4}\right): \delta 165.4,164.2$, $133.4,132.2,131.8,130.9,130.4,128.8,128.5,128.4,128.2,127.9,127.8,127.6,127.1$, $127.0,126.8,126.6,126.1,125.8,125.6,125.4,125.3,125.0,124.3,124.1,123.8,122.8$, 122.6, 122.2, 121.5, 121.3, 119.3, 54.7, 32.8, 32.5, 31.8, 26.7, 22.4, 13.8. HRMS (MALDI, 100\%): $m / z$ calcd (\%) for $\mathrm{C}_{98} \mathrm{H}_{78} \mathrm{~N}_{2} \mathrm{O}_{4}: 1347.5995$, found: $1347.5996 .[\alpha]_{\mathrm{D}}^{27}=+7316^{\circ}$.
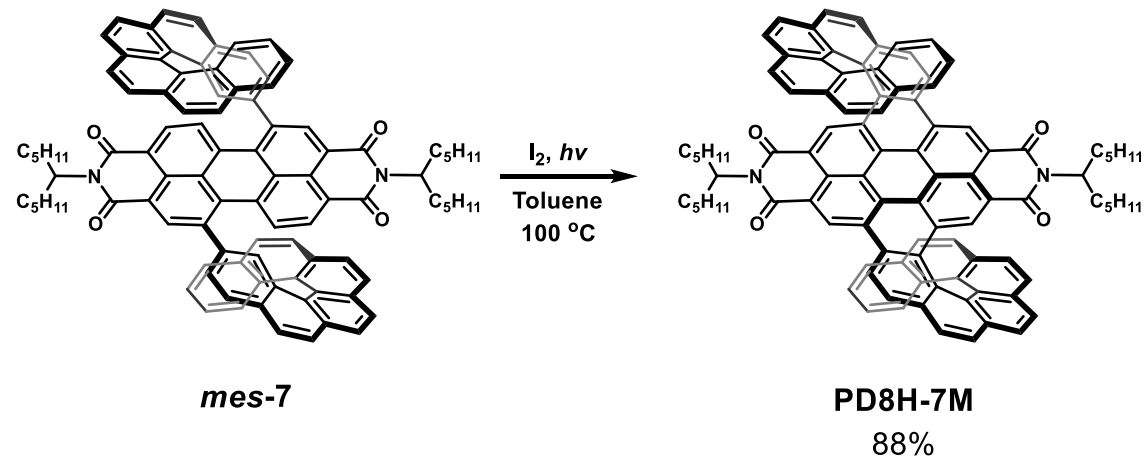

Scheme S7. Synthetic Routes to PD8H-7M 
To a standard photocyclization glassware were added $300 \mathrm{mg}$ compound $\boldsymbol{m e s}-\mathbf{7}(10 \times 30$ $\mathrm{mg}), 500 \mathrm{~mL}$ toluene $(10 \times 50 \mathrm{~mL})$ and $5 \mathrm{mg} \mathrm{I}_{2}$. The mixture was irradiated by $40 \mathrm{~W}$ LED lamp at $100{ }^{\circ} \mathrm{C}$ for three days. After the reaction, the mixture was concentrated under reduced pressure. The crude product was purified by recrystallized from $\mathrm{CHCl}_{3} / \mathrm{MeOH}(2: 1)$ to afford the title compound PD8H-7M (264 mg, 88\%) as a red solid. ${ }^{1} \mathrm{H}$ NMR (500 MHz, $373.2 \mathrm{~K}$, $\left.\mathrm{C}_{2} \mathrm{D}_{2} \mathrm{Cl}_{4}\right): \delta 10.12(\mathrm{~s}, 2 \mathrm{H}), 9.06(\mathrm{~d}, J=8.4 \mathrm{~Hz}, 2 \mathrm{H}), 8.88(\mathrm{~s}, 2 \mathrm{H}), 8.49(\mathrm{~d}, J=8.4 \mathrm{~Hz}, 2 \mathrm{H})$, $8.40(\mathrm{dd}, J=12.3,8.1 \mathrm{~Hz}, 4 \mathrm{H}), 8.33(\mathrm{~d}, J=8.1 \mathrm{~Hz}, 2 \mathrm{H}), 8.20(\mathrm{~d}, J=8.1 \mathrm{~Hz}, 2 \mathrm{H}), 7.76(\mathrm{~d}, J=$ $8.0 \mathrm{~Hz}, 2 \mathrm{H}), 7.06(J=8.0 \mathrm{~Hz}, 2 \mathrm{H}), 6.92(\mathrm{~d}, J=5.2 \mathrm{~Hz}, 2 \mathrm{H}), 6.34(\mathrm{dd}, J=5.7,2.2 \mathrm{~Hz}, 4 \mathrm{H})$, $5.66(\mathrm{~d}, J=8.0 \mathrm{~Hz}, 2 \mathrm{H}), 5.43(\mathrm{~s}, 2 \mathrm{H}), 5.29(\mathrm{~s}, 2 \mathrm{H}), 4.92(\mathrm{~d}, J=8.5 \mathrm{~Hz}, 2 \mathrm{H}), 2.55(\mathrm{~s}, 4 \mathrm{H}), 2.13$ (s, 4H), 1.58 (s, 24H), 1.00 (s, 12H). ${ }^{13} \mathrm{C}$ NMR (125 MHz, $\left.373.2 \mathrm{~K}, \mathrm{C}_{2} \mathrm{D}_{2} \mathrm{Cl}_{4}\right): \delta 133.2,132.0$, $131.7,130.6,129.7,128.6,128.3,128.1,127.6,127.2,127.0,126.6,126.3,125.7,125.5$, $124.7,124.5,124.2,123.9,123.8,123.5,122.4,122.0,121.5,121.2,54.7,32.9,32.5,31.7$, 26.7, 22.5, 13.8. HRMS (MALDI, 100\%): $\mathrm{m} / z$ calcd (\%) for $\mathrm{C}_{98} \mathrm{H}_{78} \mathrm{~N}_{2} \mathrm{O}_{4}:$ 1347.5995, found: 1347.6001 .

\section{Optical, Electrochemical, and Thermal Properties}

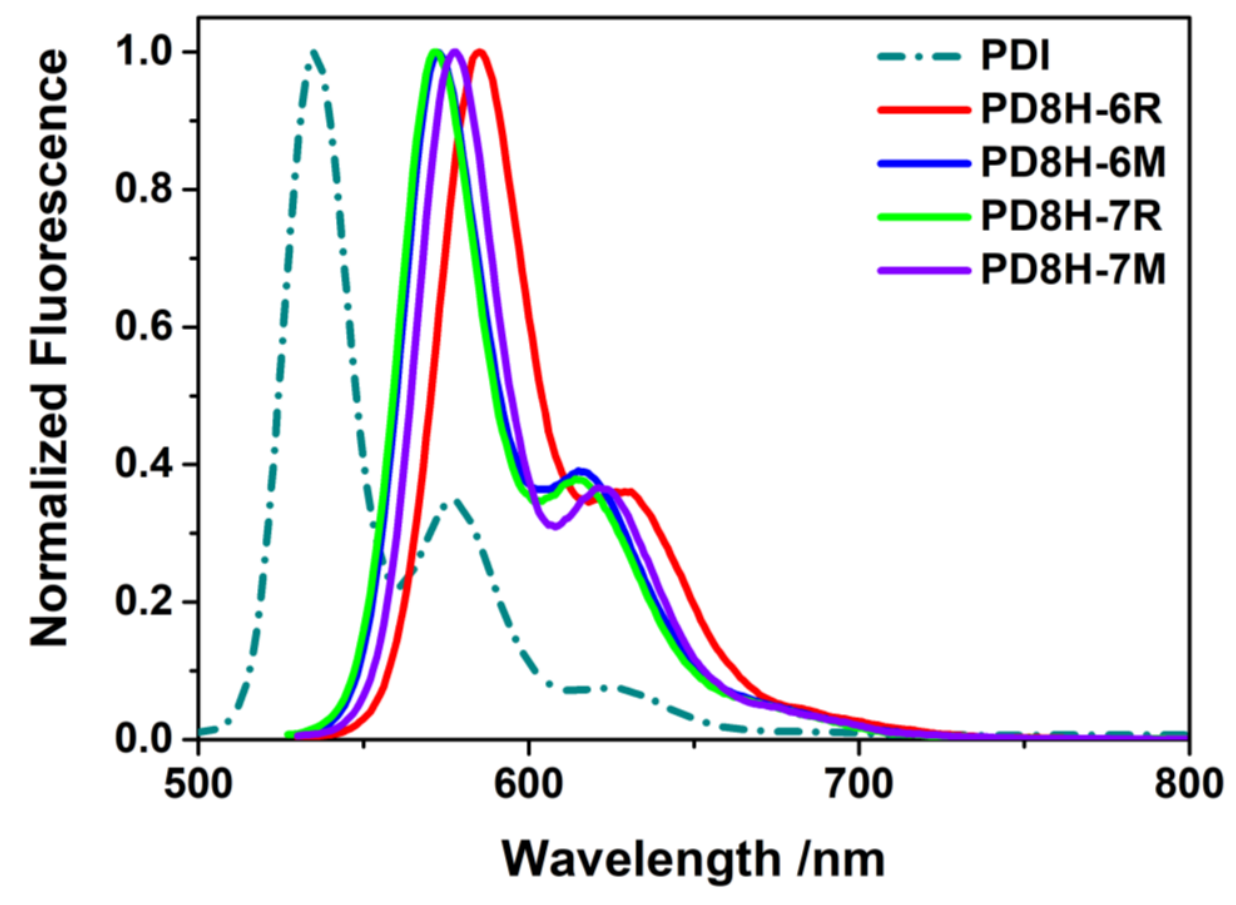

Figure S1. Normalized fluorescence emission spectra in $\mathrm{CHCl}_{3}\left(1 \times 10^{-5} \mathrm{M}\right)$. 


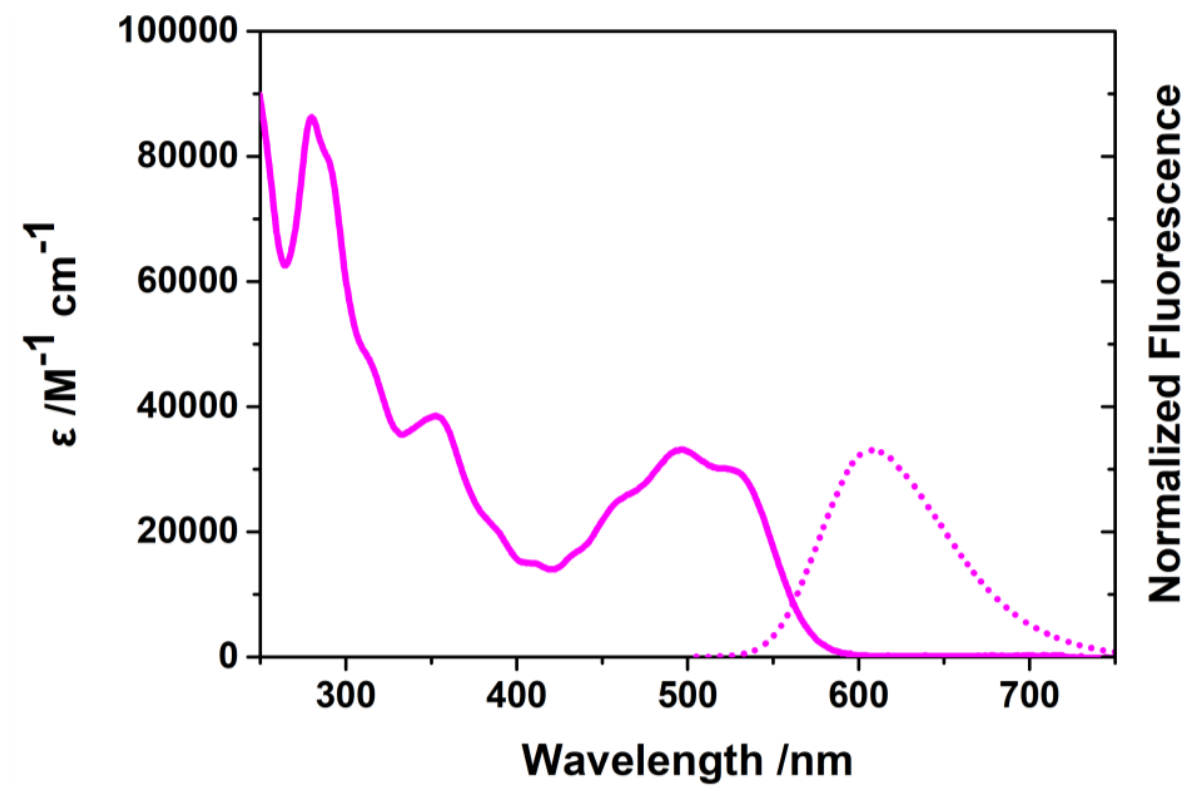

Figure S2. UV-Vis absorption and normalized fluorescence emission spectra of $\mathbf{P 8 H}$ in $\mathrm{CHCl}_{3}(1$ $\left.\times 10^{-5} \mathrm{M}\right)$.

a)
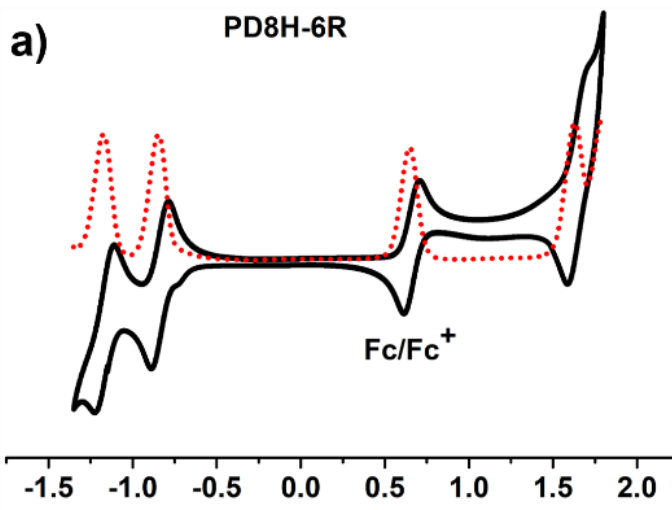

Potential /V

c)

PD8H-7R
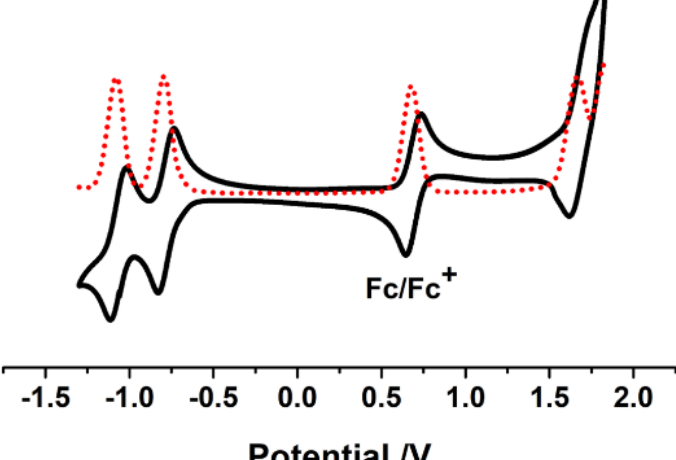

b)

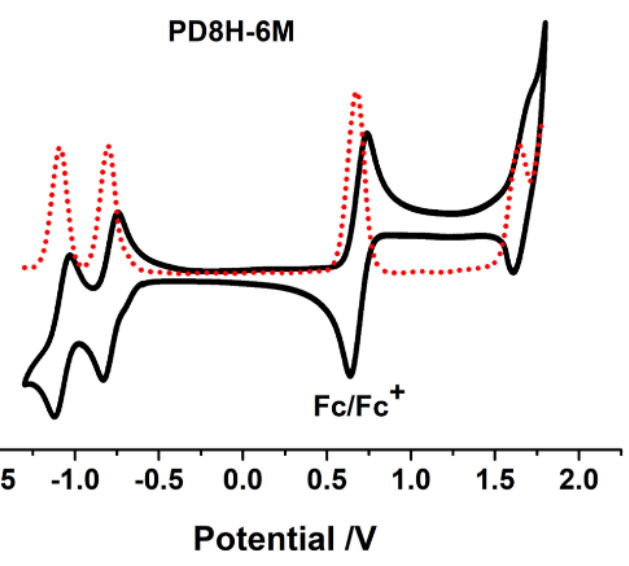

d)
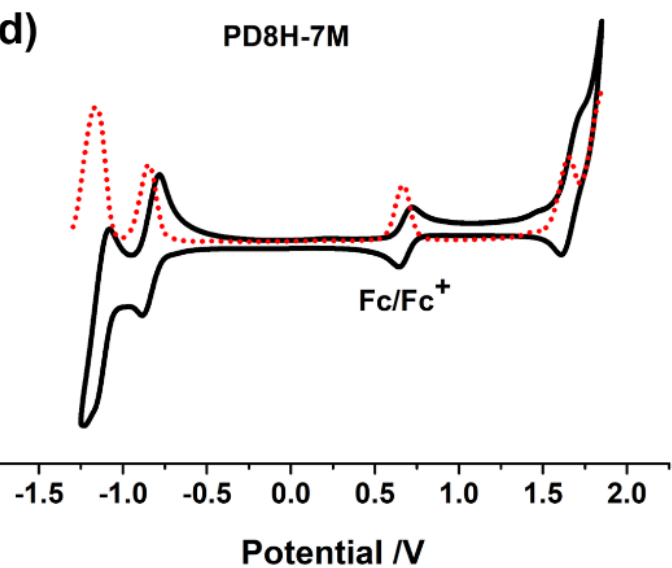

Figure S3. Cyclic voltammetry (black solid trace) and differential pulse voltammetry (red dot trace) profiles of PD8H-6R (a), PD8H-6M (b), PD8H-7R (c) and PD8H-7M (d). 


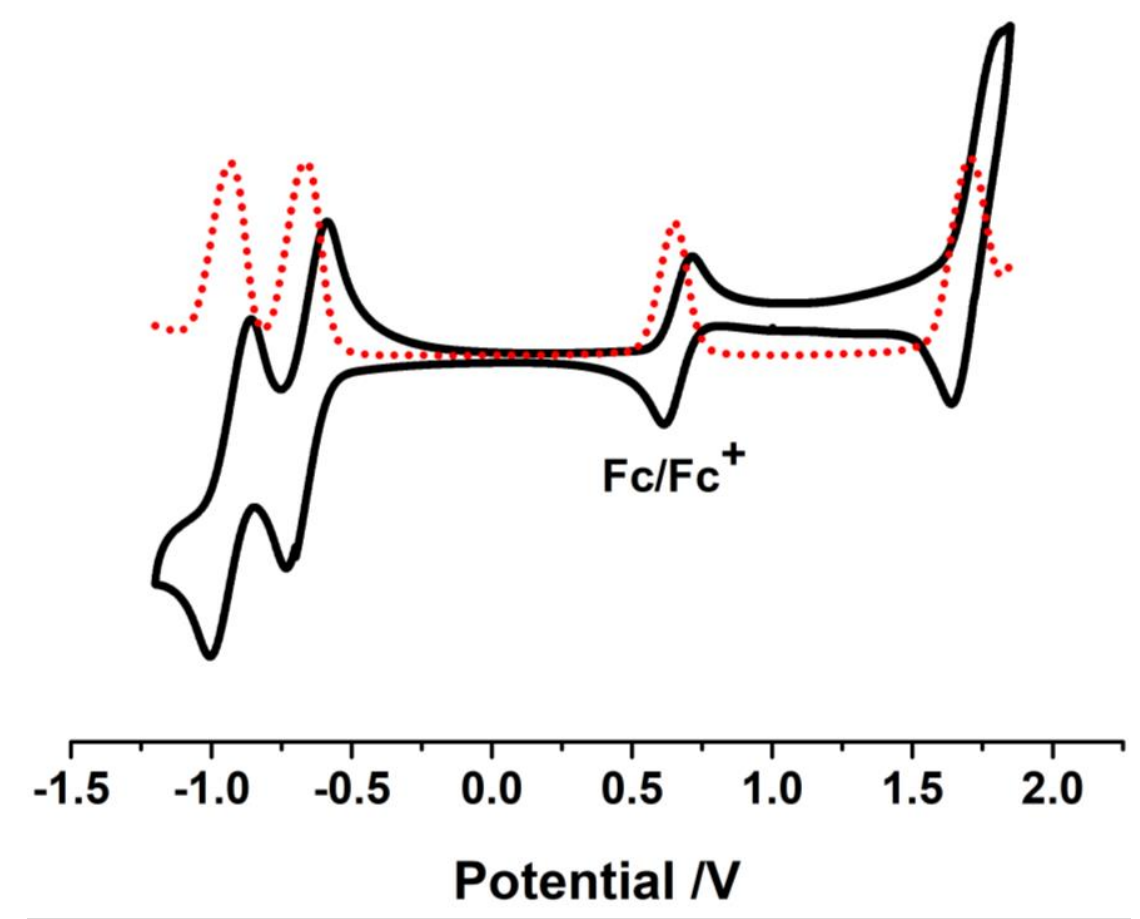

Figure S4. Cyclic voltammetry (black solid trace) and differential pulse voltammetry (red dot trace) profiles of $\mathbf{P 8 H}$.

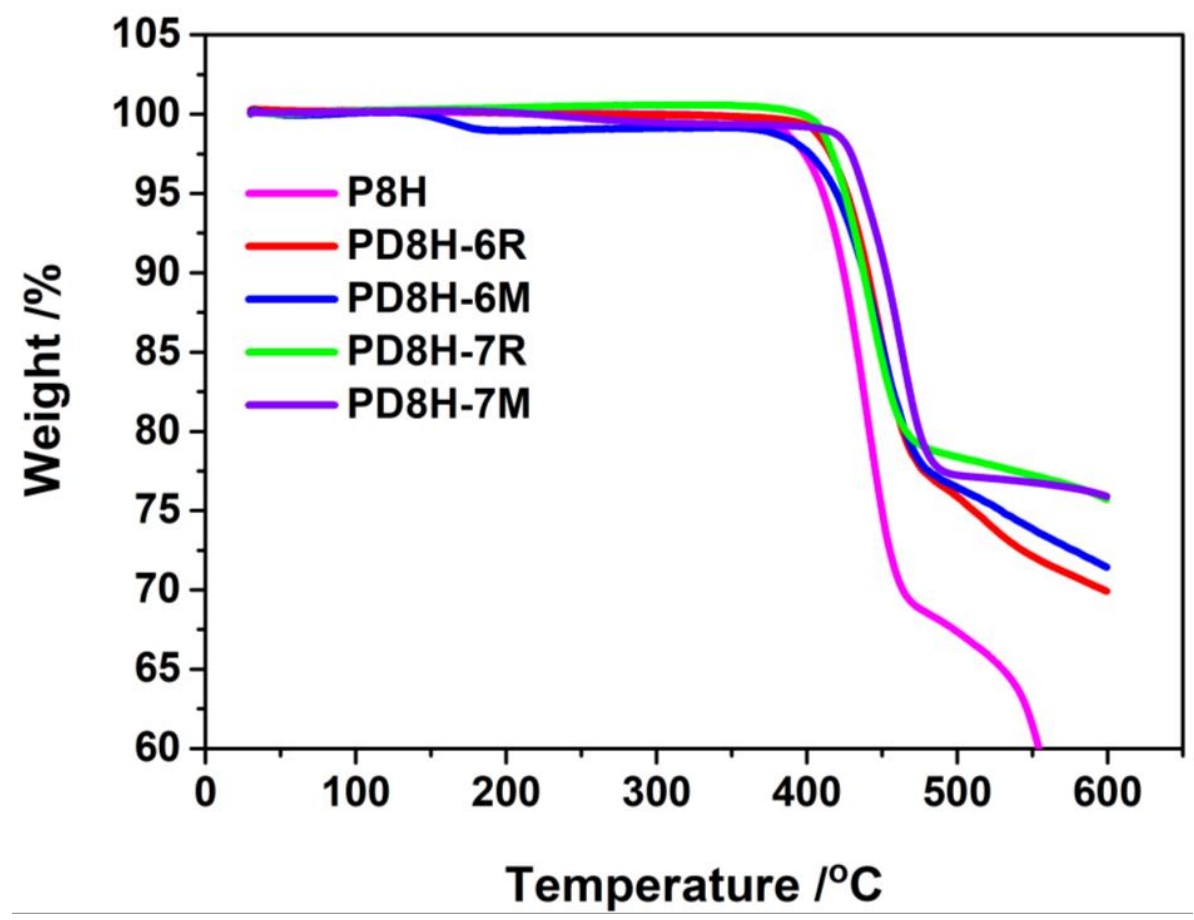

Figure S5. TGA curves under nitrogen flow. 
Table S1. Summary of optical properties of P8H and PD8Hs in solutions and films

\begin{tabular}{|c|c|c|c|c|c|c|c|}
\hline & $\begin{array}{l}\lambda_{\mathrm{abs}}{ }^{\mathrm{sol}} . \\
{[\mathrm{nm}]^{a}}\end{array}$ & $\begin{array}{c}\varepsilon_{\max }{ }^{\mathrm{sol} .} \\
{\left[\mathrm{M}^{-1} \mathrm{~cm}^{-1}\right]^{a}}\end{array}$ & $\begin{array}{l}\lambda_{\mathrm{em}}{ }^{\text {sol. }} \\
{[\mathrm{nm}]^{a}}\end{array}$ & $\begin{array}{l}E_{\mathrm{g}}{ }^{\text {sol. }} \\
{[\mathrm{eV}]^{b}}\end{array}$ & $\begin{array}{c}\lambda_{\mathrm{abs}}{ }^{\mathrm{film}} \\
{[\mathrm{V}]^{c}}\end{array}$ & $\begin{array}{c}\lambda_{\mathrm{em}}^{\text {film }} \\
{[\mathbf{V}]^{c}}\end{array}$ & $\begin{array}{r}E_{\mathrm{g}}^{\text {film }} \\
{[\mathbf{V}]^{d}}\end{array}$ \\
\hline P8H & $\begin{array}{l}498 \\
528\end{array}$ & $\begin{array}{l}33100 \\
29700\end{array}$ & 611 & 2.17 & $\begin{array}{l}498 \\
536\end{array}$ & 619 & 2.13 \\
\hline PD8H-6R & $\begin{array}{l}526 \\
566\end{array}$ & $\begin{array}{l}23500 \\
26300\end{array}$ & 585 & 2.11 & $\begin{array}{l}523 \\
563\end{array}$ & 593 & 2.11 \\
\hline PD8H-6M & $\begin{array}{l}518 \\
556\end{array}$ & $\begin{array}{l}21700 \\
23400\end{array}$ & 572 & 2.15 & $\begin{array}{l}520 \\
557\end{array}$ & 608 & 2.13 \\
\hline PD8H-7R & $\begin{array}{l}518 \\
557\end{array}$ & $\begin{array}{l}21900 \\
25000\end{array}$ & 572 & 2.15 & $\begin{array}{l}519 \\
557\end{array}$ & 608 & 2.12 \\
\hline PD8H-7M & $\begin{array}{l}523 \\
563\end{array}$ & $\begin{array}{l}21200 \\
25200\end{array}$ & 578 & 2.13 & $\begin{array}{l}520 \\
560\end{array}$ & 578 & 2.13 \\
\hline
\end{tabular}

${ }^{a}$ Measured in a dilute $\mathrm{CHCl}_{3}$ solution $\left(1.0 \times 10^{-5} \mathrm{M}\right) .{ }^{b}$ Calculated by the onset of absorption in a $\mathrm{CHCl}_{3}$ solution according to $E_{\mathrm{g}}{ }^{\text {opt }}(\mathrm{eV})=\left(1240 / \lambda_{\text {onset }}\right) .{ }^{c}$ Measured in film. ${ }^{d}$ Calculated by the onset of absorption in film.

Table S2. Summary of electrochemical properties, thermal properties, and energy gaps of P8H and PD8Hs with PDI as reference

\begin{tabular}{|c|c|c|c|c|c|c|c|c|}
\hline & $\begin{array}{c}E_{\mathrm{g}}{ }^{\mathrm{opt}} \\
{[\mathrm{eV}]^{a}}\end{array}$ & $\begin{array}{c}E_{1 \mathbf{r}} \\
{[\mathrm{V}]^{b}}\end{array}$ & $\begin{array}{c}E_{2 \mathbf{r}} \\
{[\mathrm{V}]^{b}}\end{array}$ & $\begin{array}{c}E_{10} \\
{[\mathrm{~V}]^{b}}\end{array}$ & $\begin{array}{c}E_{\text {Lumo }} \\
{[\mathrm{eV}]^{c}}\end{array}$ & $\begin{array}{c}\boldsymbol{E}_{\text {номо }} \\
{[\mathrm{eV}]^{d}}\end{array}$ & $\begin{array}{c}\boldsymbol{E}_{\mathrm{g}}{ }^{\text {electro }} \\
{[\mathrm{eV}]^{f}}\end{array}$ & $\begin{array}{r}\boldsymbol{T}_{\mathrm{deg}} \\
\left.{ }^{\circ} \mathbf{C}\right]^{g}\end{array}$ \\
\hline PDI & 2.30 & -1.10 & -1.30 & --- & -3.86 & $-6.16^{e}$ & --- & 403 \\
\hline P8H & 2.17 & -1.32 & -1.60 & 1.06 & -3.63 & -5.83 & 2.20 & 411 \\
\hline PD8H-6R & 2.11 & -1.50 & -1.83 & 0.99 & -3.44 & -5.75 & 2.31 & 427 \\
\hline PD8H-6M & 2.15 & -1.48 & -1.76 & 0.97 & -3.48 & -5.73 & 2.25 & 420 \\
\hline PD8H-7R & 2.15 & -1.47 & -1.76 & 0.99 & -3.48 & -5.76 & 2.28 & 426 \\
\hline PD8H-7M & 2.13 & -1.52 & -1.81 & 0.98 & -3.42 & -5.75 & 2.33 & 439 \\
\hline
\end{tabular}

${ }^{a}$ Calculated by the onset of absorption in a $\mathrm{CHCl}_{3}$ solution according to $E_{\mathrm{g}}{ }^{\text {opt }}(\mathrm{eV})=\left(1240 / \lambda_{\text {onset }}\right){ }^{b}$ Half-wave reductive and oxidative potentials (in $\mathrm{V} v \mathrm{Fc} / \mathrm{Fc}^{+}$) measured in $\mathrm{CH}_{2} \mathrm{Cl}_{2}$ at a scan rate of $0.1 \mathrm{~V} / \mathrm{s}$ using ferrocene as an internal potential mark. ${ }^{c} \mathrm{LUMO}(\mathrm{eV})$ estimated by the onset of the reduction peaks and calculated according to $E_{\mathrm{LUMO}}=-\left(4.8+E_{\text {onset }^{\mathrm{re}}}\right) \mathrm{eV} .{ }^{d} \mathrm{HOMO}$ estimated by the onset of the oxidative peaks and calculated according to $E_{\text {Hомо }}=-\left(4.8+E_{\text {onset }{ }^{\mathrm{ox}}}\right) \mathrm{eV} \cdot{ }^{e} \mathrm{HOMO}(\mathrm{eV})$ calculated according to $E_{\text {Hомо }}=\left(E_{\mathrm{LumO}}-E_{\mathrm{g}}{ }^{\mathrm{opt}}\right) \mathrm{eV} \cdot{ }^{f}$ Calculated according to $E_{\mathrm{g}}{ }^{\text {lectro }}=\left(E_{\mathrm{LUMO}}-E_{\mathrm{HOMO}}\right) \mathrm{eV} \cdot{ }^{g}$ Decomposition temperature determined by TGA corresponding to $5 \%$ weight loss at $10^{\circ} \mathrm{C} / \mathrm{min}$ under a nitrogen flow. 
Table S3. The fluorescence quantum yields and corresponding singlet lifetime of PD8Hs

\begin{tabular}{ccccc}
\hline & $\begin{array}{c}\Phi_{\mathrm{fl}} \\
{[\%]^{a}}\end{array}$ & $\begin{array}{c}\boldsymbol{\tau}_{\mathrm{s}} \\
{[\mathrm{ns}]^{b}}\end{array}$ & $\begin{array}{c}\boldsymbol{k}_{\mathrm{r}} \\
{\left[\mathrm{s}^{-1}\right]^{c}}\end{array}$ & $\begin{array}{c}\boldsymbol{k}_{\mathrm{nr}} \\
{\left[\mathrm{s}^{-1}\right]^{c}}\end{array}$ \\
\hline PD8H-6R & 33 & 7.8 & $4.2 \times 10^{7}$ & $8.6 \times 10^{7}$ \\
PD8H-6M & 29 & 7.4 & $3.9 \times 10^{7}$ & $9.6 \times 10^{7}$ \\
PD8H-7R & 31 & 7.8 & $4.0 \times 10^{7}$ & $8.8 \times 10^{7}$ \\
PD8H-7M & 34 & 8.1 & $4.2 \times 10^{7}$ & $8.1 \times 10^{7}$ \\
\hline
\end{tabular}

${ }^{a}$ Measured in a dilute $\mathrm{CHCl}_{3}$ solution $\left(1.0 \times 10^{-5} \mathrm{M}\right)$ and determined by absolute quantum yield method. ${ }^{b}$ The fluorescence lifetime values. ${ }^{c}$ The radiative $\left(k_{\mathrm{r}}\right)$ and nonradiative $\left(k_{\mathrm{nr}}\right)$ decay rate constants from the singlet excited state are determined by the equations $\Phi_{\mathrm{fl}}=k_{\mathrm{r}} \times \tau_{\mathrm{s}}$ and $k_{\mathrm{r}}+k_{\mathrm{nr}}=\tau_{\mathrm{s}}^{-1}$.

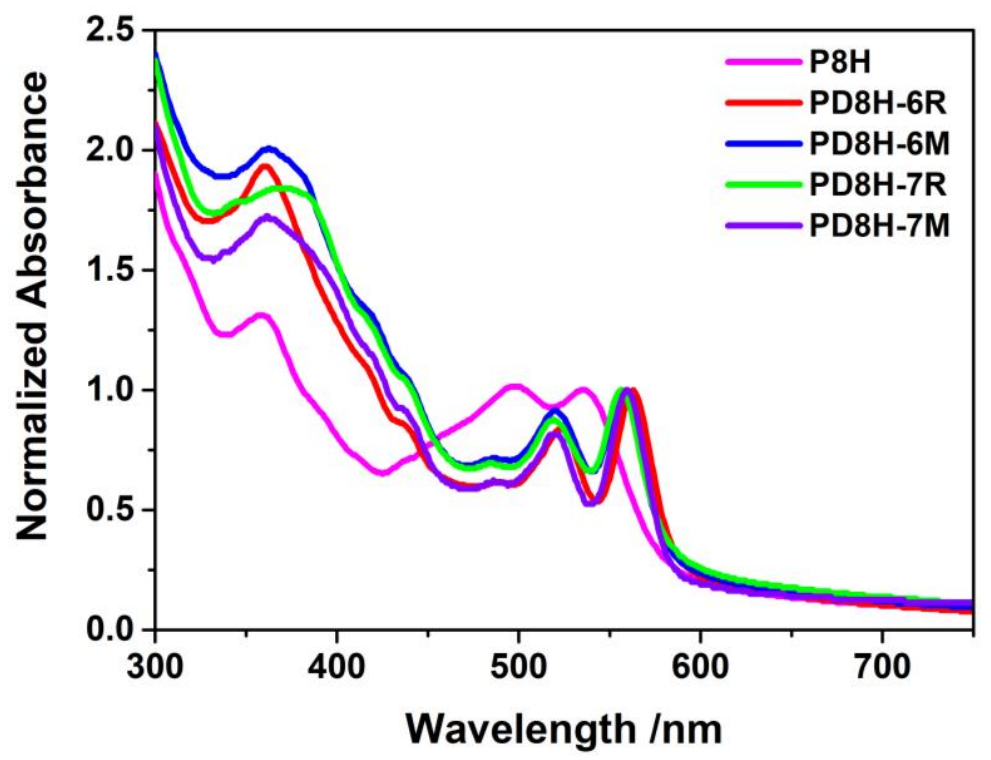

Figure S6. Normalized UV-Vis absorption spectra of P8H and PD8Hs in films.

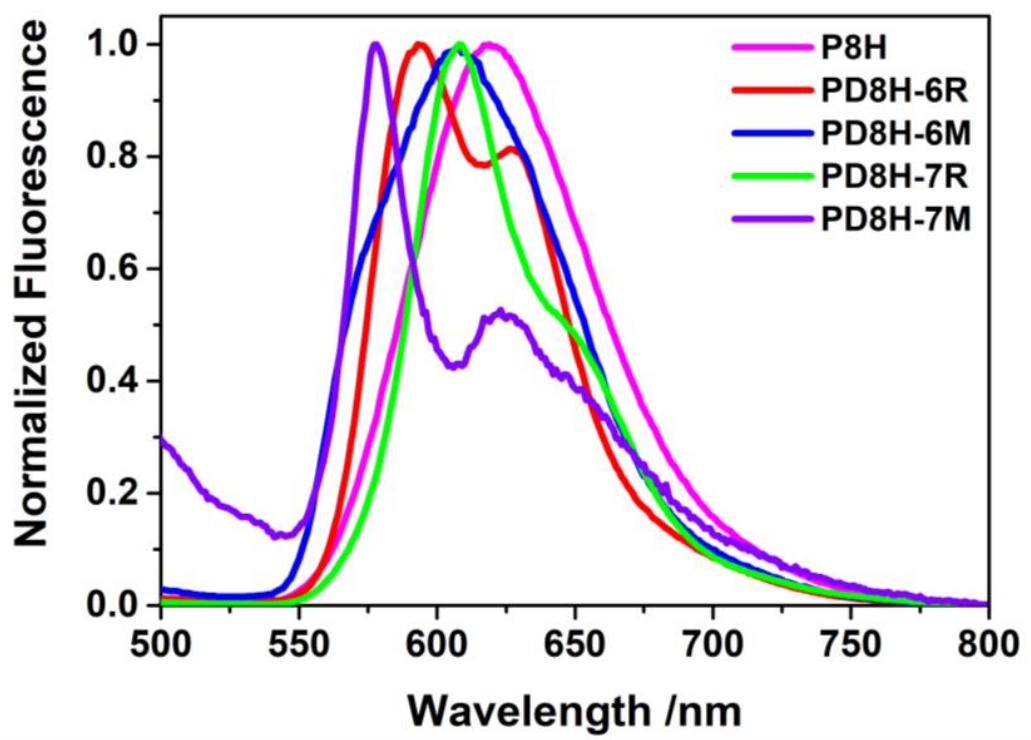

Figure S7. Normalized fluorescence emission spectra of P8H and PD8Hs in films. 


\section{Chiral HPLC Chromatogram}

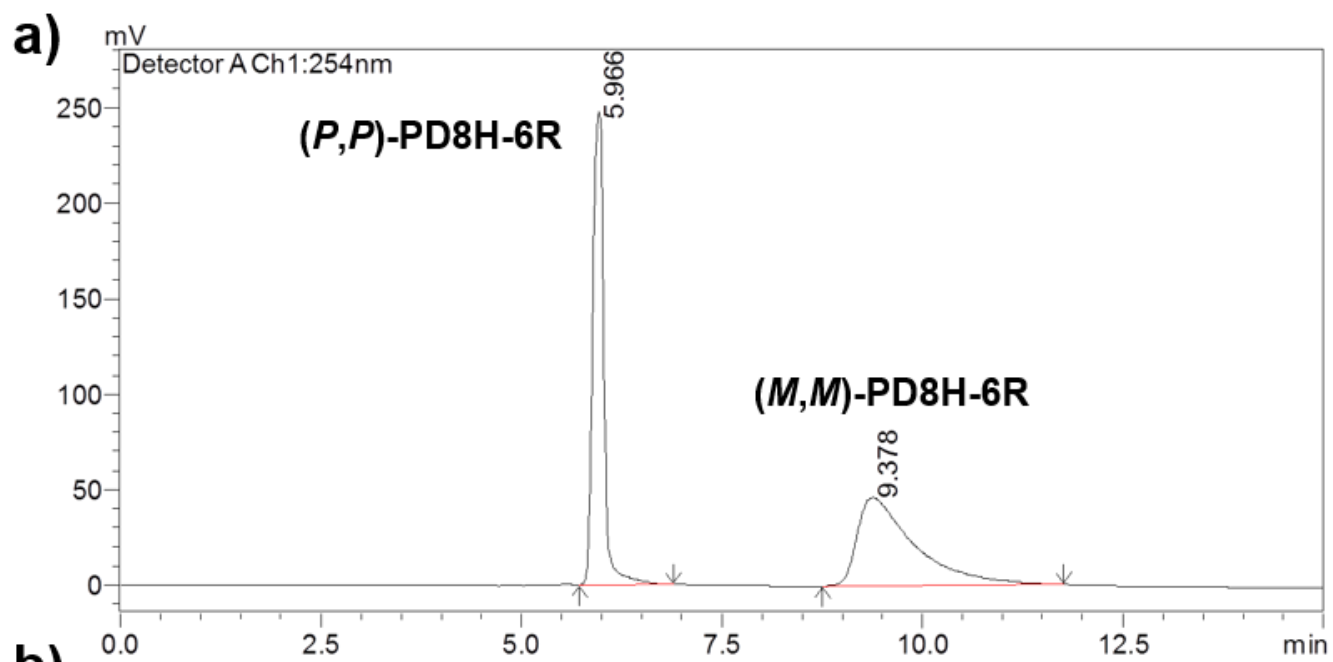

b)

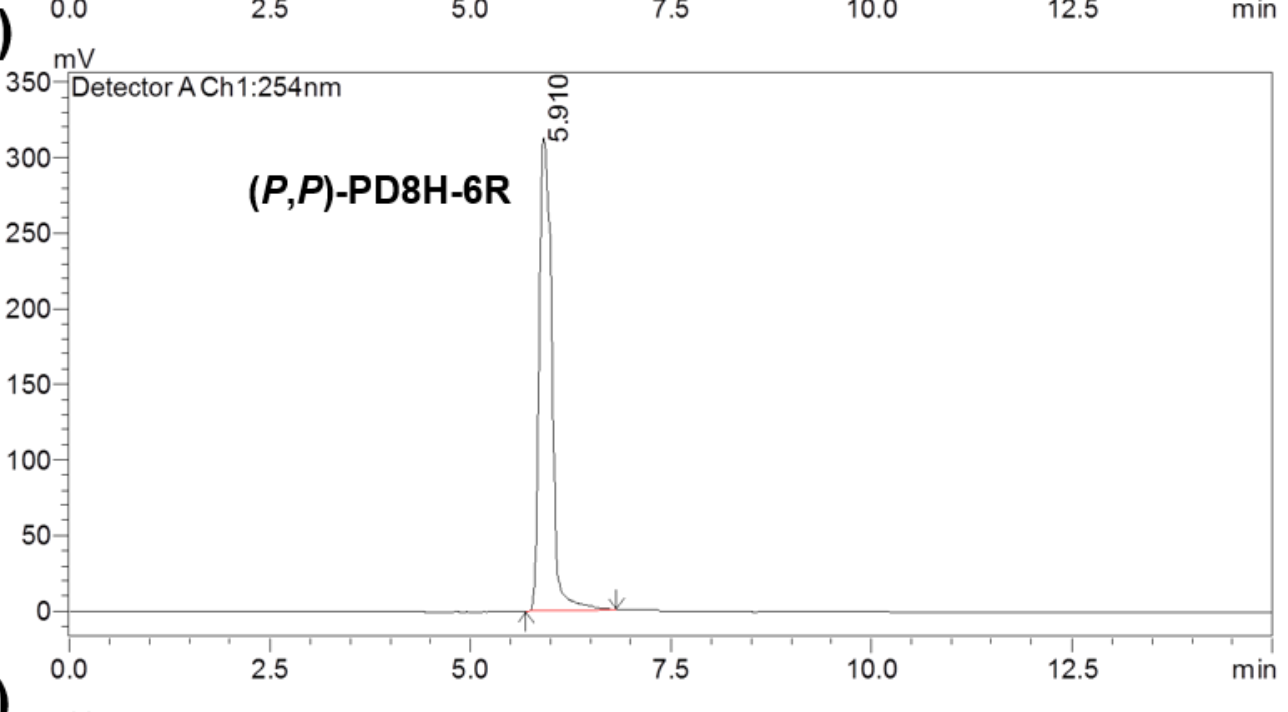

c)

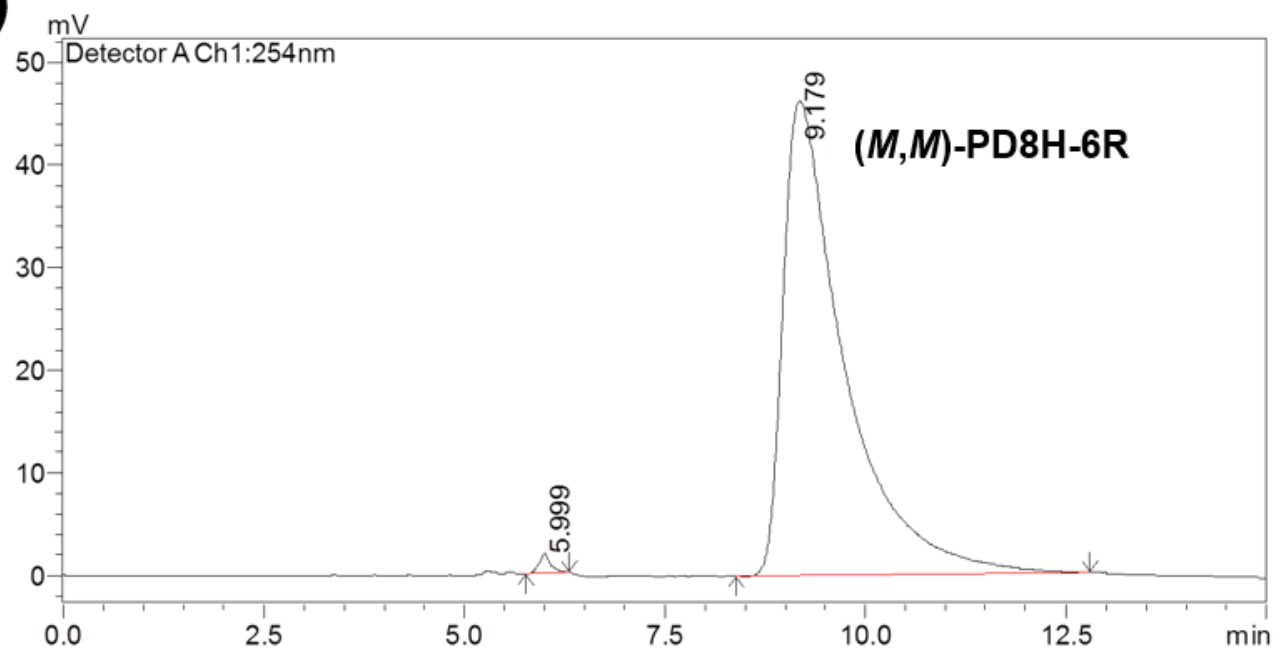

Figure S8. Resolution of PD8H-6R by chiral HPLC monitored at $254 \mathrm{~nm}$ was performed with a CHIRALPAK IE- 3 column $(0.46 \mathrm{~cm}$ I.D. $\times 25 \mathrm{~cm} \mathrm{~L})$. Injection volume was $1 \mu \mathrm{L}$, and a mixture of Hexane $/ \mathrm{CH}_{2} \mathrm{Cl}_{2}=51 / 49(\mathrm{~V} / \mathrm{V})$ was used as the eluent with a flow rate of $1.0 \mathrm{~mL} / \mathrm{min}$ at $35^{\circ} \mathrm{C}$. 


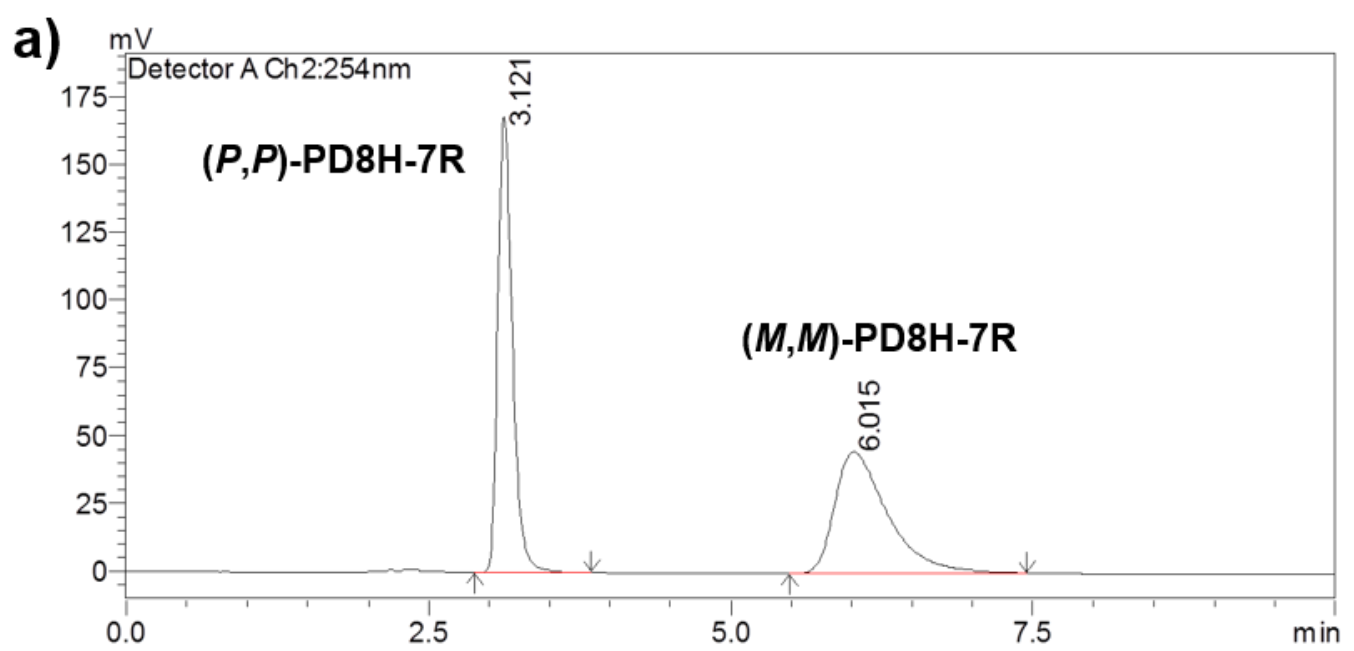

b)

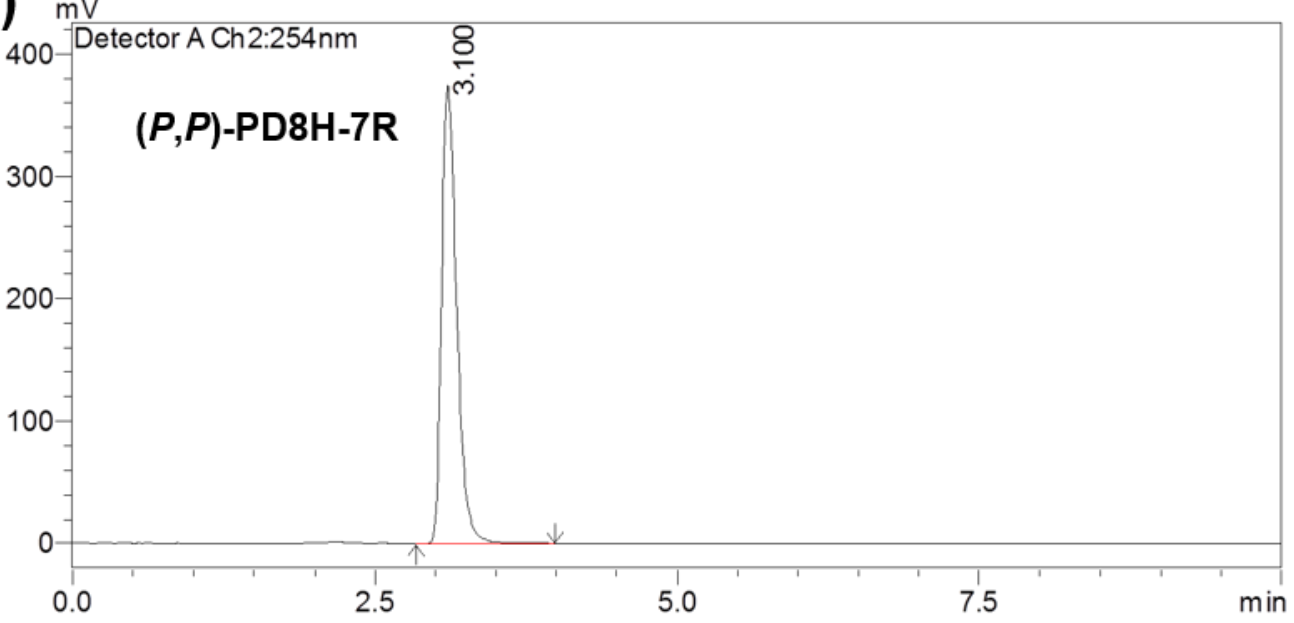

c)

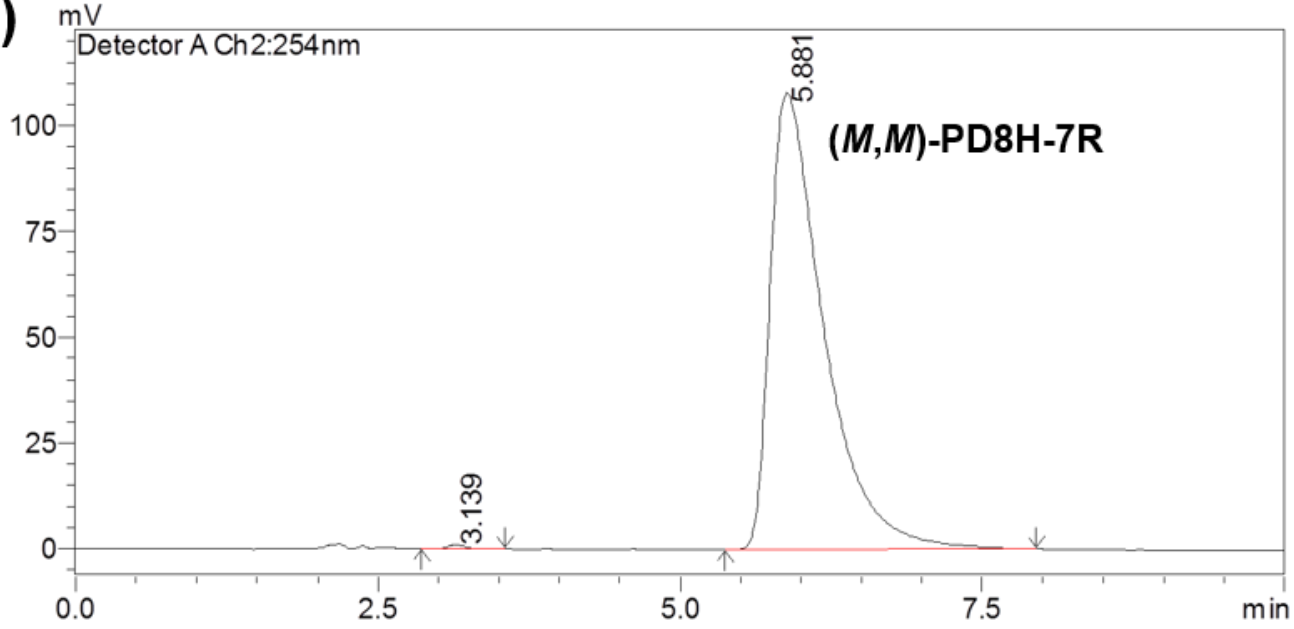

Figure S9. Resolution of PD8H-7R by chiral HPLC monitored at $254 \mathrm{~nm}$ was performed with a CHIRALPAK IC $(0.46 \mathrm{~cm} \mathrm{I.D.} \times 15 \mathrm{~cm} \mathrm{~L})$. Injection volume was $2 \mu \mathrm{L}$, and a mixture of $\mathrm{Hexane} / \mathrm{EtOH}=50 / 50(\mathrm{~V} / \mathrm{V})$ was used as the eluent with a flow rate of $1.0 \mathrm{~mL} / \mathrm{min}$ at $25^{\circ} \mathrm{C}$. 


\section{CD and CPL Spectra}
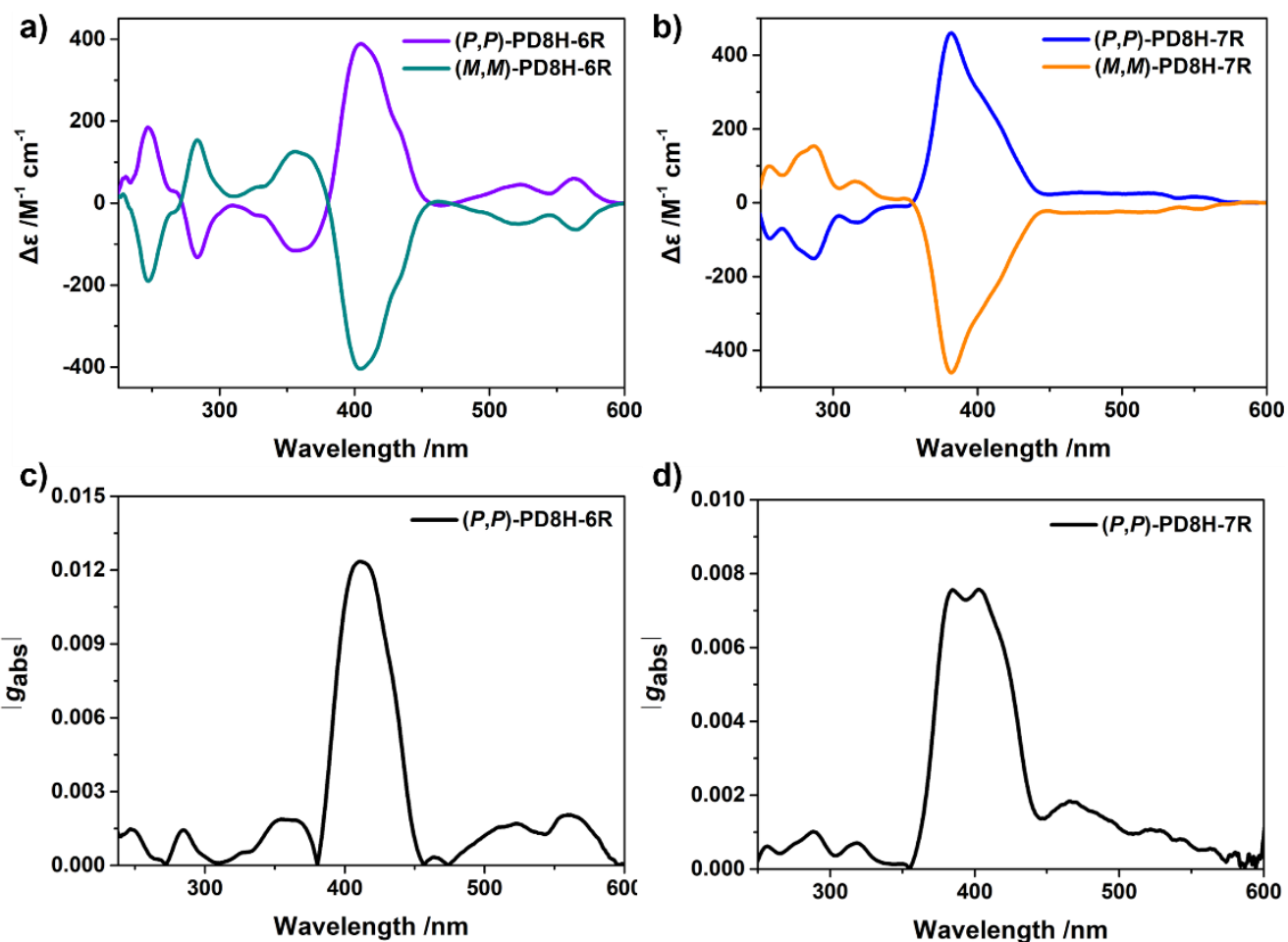

Figure S10. CD spectra (a, b) and the corresponding $\left|g_{\text {abs }}\right|$ factors of the first fractions (c, d) of PD8H-6R (a, c) and PD8H-7R (b, d) in $\mathrm{CHCl}_{3}\left(1 \times 10^{-5} \mathrm{M}\right)$.
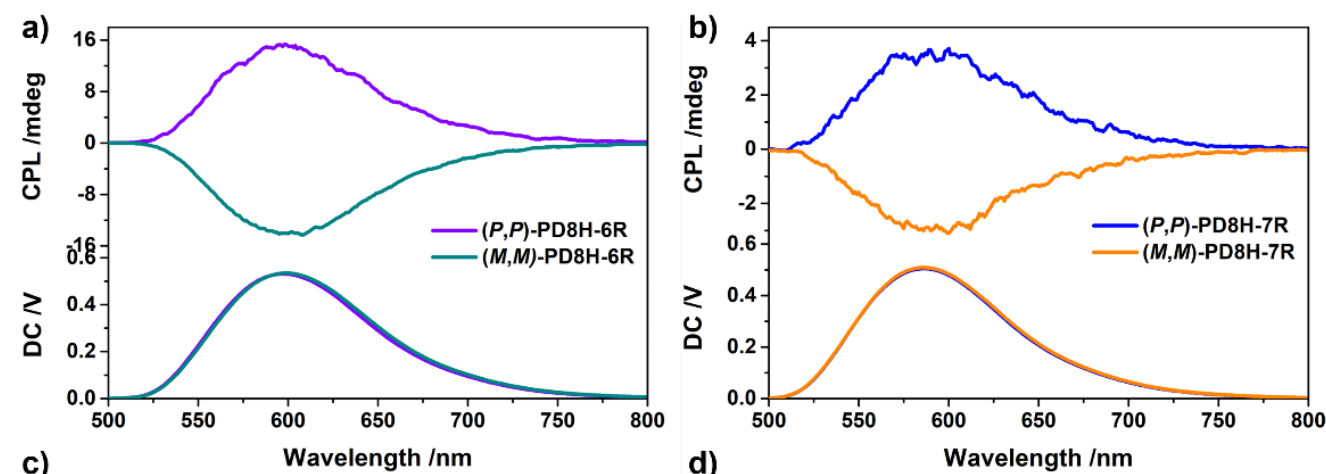

c)

d)
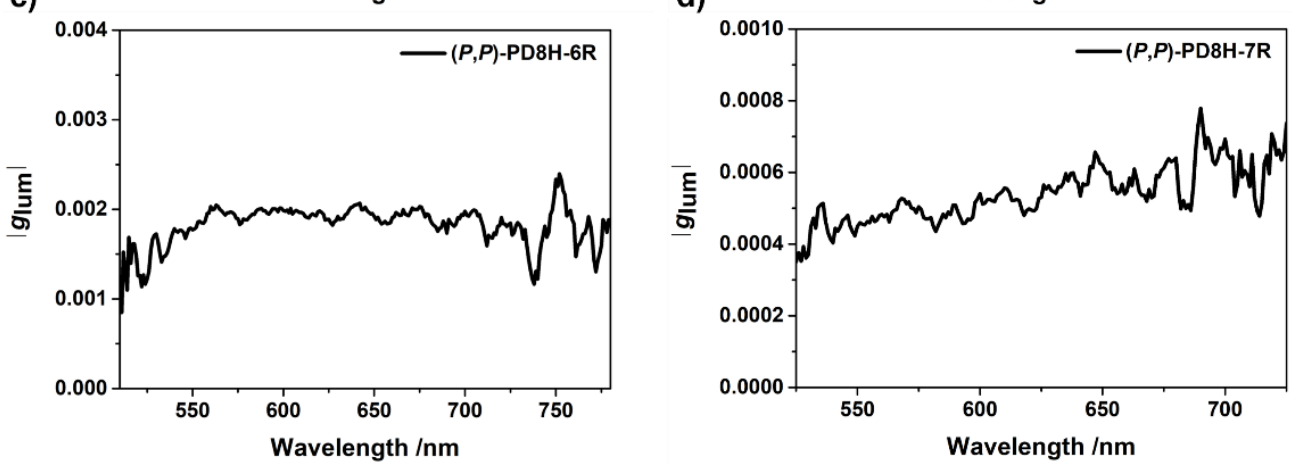

Figure S11. CPL spectra (a, b) and the corresponding $\left|g_{\text {lum }}\right|$ factors of the first fractions (c, d) of PD8H-6R (a, c) and PD8H-7R (b, d) excited at $360 \mathrm{~nm}$ in $\mathrm{CHCl}_{3}\left(1 \times 10^{-5} \mathrm{M}\right)$. 
a)

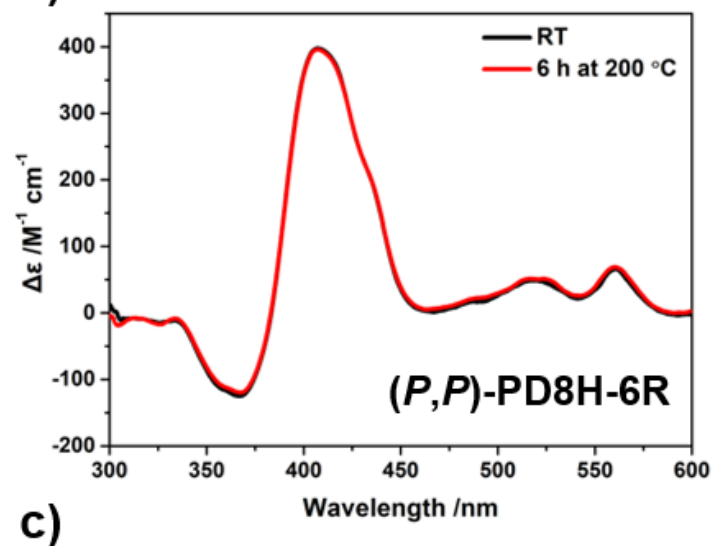

b)

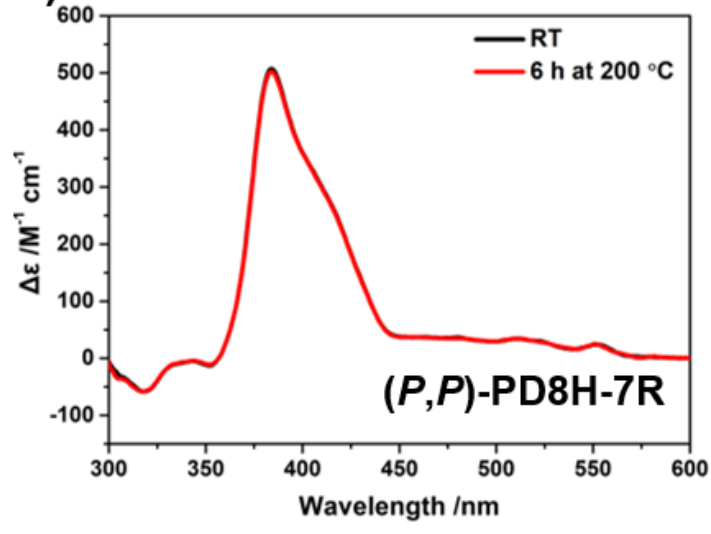

$(P, P)$-PD8H-6R
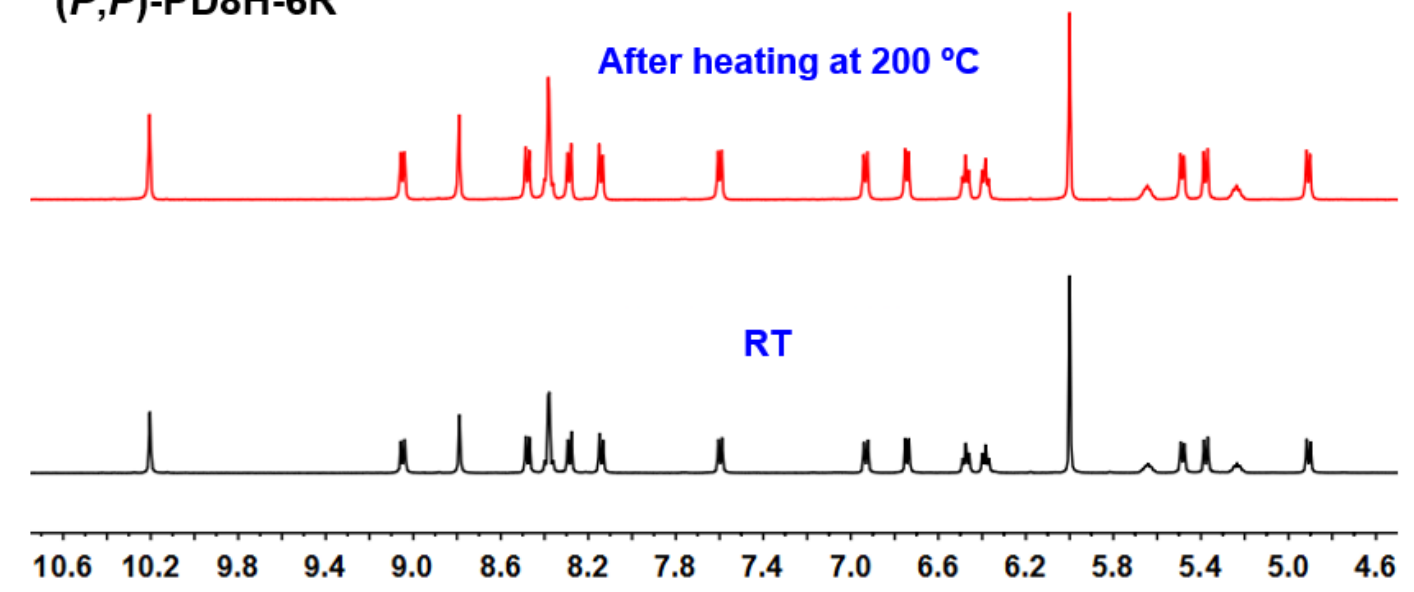

d) $\delta$ (ppm)

\section{$(P, P)$-PD8H-7R}

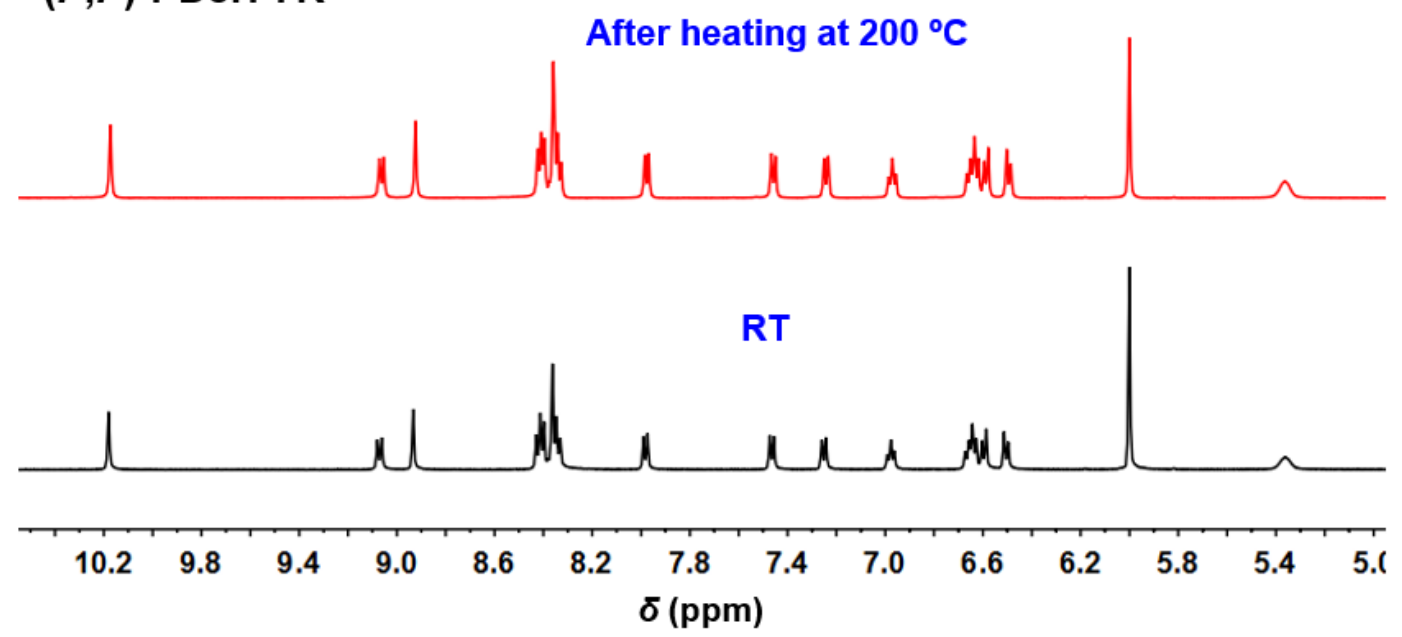

Figure S12. (a) CD spectra and (b) ${ }^{1} \mathrm{H}$ NMR spectra (500 MHz, $373.2 \mathrm{~K}, \mathrm{C}_{2} \mathrm{D}_{2} \mathrm{Cl}_{4}$ ) of the first fractions of PD8H-6R and PD8H-7R before heating (RT) and after heating at $200{ }^{\circ} \mathrm{C}$ for 6 hours in diphenyl ether solution for comparison. 


\section{X-ray Crystal Structure}

Single crystals suitable for X-ray analysis were grown at room temperature by slow evaporation of methanol into a dichloroethane solution for $\mathbf{P 8 H}$, by slow evaporation of methanol into a $\mathrm{CHCl}_{3}$ solution for PD8H-6R, $(P, P)$-PD8H-6R, $(M, M)$-PD8H-6R, PD8H-7R and PD8H-7M as well as by slow evaporation of methanol into a toluene solution for PD8H-6M.

Crystallographic data have been deposited with the Cambridge Crystallographic Data Centre as supplementary publication on No. CCDC 1974307 (P8H), No. CCDC 1974308 (PD8H-6R), No. CCDC 1974309 (PD8H-6M), No. CCDC 1974310 (PD8H-7R), No. CCDC 1974311 (PD8H-7M), No. CCDC 1974313 ((P,P)-PD8H-6R), and No. CCDC 1974312 ((M,M)-PD8H-6R). The crystallographic data were summarized below.

Table S4. Crystal data and structure refinement for P8H

\begin{tabular}{|c|c|}
\hline Empirical formula & $\mathrm{C}_{72} \mathrm{H}_{66} \mathrm{~N}_{2} \mathrm{O}_{4}$ \\
\hline Formula weight & 1023.26 \\
\hline $\mathrm{T}(\mathrm{K})$ & $169.99(10)$ \\
\hline Crystal system & triclinic \\
\hline Space group & $\mathrm{P}-1$ \\
\hline a $(\AA)$ & $15.1377(2)$ \\
\hline $\mathrm{b}(\AA)$ & $19.4899(3)$ \\
\hline c $(\AA)$ & $22.4137(2)$ \\
\hline$\alpha(\operatorname{deg})$ & $106.7040(10)$ \\
\hline$\beta(\operatorname{deg})$ & $98.6010(10)$ \\
\hline$\gamma(\operatorname{deg})$ & $105.0170(10)$ \\
\hline $\mathrm{V}\left(\AA^{3}\right)$ & 5935.33(14) \\
\hline $\mathrm{Z}$ & 4 \\
\hline$D_{\text {calc }}\left(\mathrm{g} / \mathrm{cm}^{3}\right)$ & 1.145 \\
\hline$\mu\left(\mathrm{mm}^{-1}\right)$ & 0.544 \\
\hline $\mathrm{F}(000)$ & 2176.0 \\
\hline Crystal size $\left(\mathrm{mm}^{3}\right)$ & $0.33 \times 0.22 \times 0.1$ \\
\hline Radiation & $\mathrm{CuK} \alpha(\lambda=1.54184)$ \\
\hline$\Theta$ range (deg) & $2.49-66.50$ \\
\hline Reflns collected & 206866 \\
\hline Indep reflns / $\mathrm{R}_{\text {int }}$ & $20858 / 0.0838$ \\
\hline Data/restraints/parameters & $20858 / 115 / 1409$ \\
\hline GOF on $F^{2}$ & 1.717 \\
\hline
\end{tabular}




\begin{tabular}{|c|c|}
\hline $\mathrm{R}_{1}, \mathrm{w} R_{2}[\mathrm{I}>=2 \sigma(\mathrm{I})]$ & $0.1110 / 0.3620$ \\
\hline $\mathrm{R}_{1}, \mathrm{w} R_{2}$ [all data] & $0.1216 / 0.3817$ \\
\hline
\end{tabular}

Table S5. Crystal data and structure refinement for PD8H-6R and PD8H-6M

\begin{tabular}{|c|c|c|}
\hline Compound & PD8H-6R & PD8H-6M \\
\hline Empirical formula & $\mathrm{C}_{98} \mathrm{H}_{78} \mathrm{~N}_{2} \mathrm{O}_{4}$ & $\mathrm{C}_{392} \mathrm{H}_{312} \mathrm{~N}_{8} \mathrm{O}_{16}$ \\
\hline Formula weight & 1347.62 & 5390.48 \\
\hline $\mathrm{T}(\mathrm{K})$ & $169.99(10)$ & $169.99(13)$ \\
\hline Crystal system & triclinic & triclinic \\
\hline Space group & $\mathrm{P}-1$ & $\mathrm{P}-1$ \\
\hline $\mathrm{a}(\AA)$ & $7.84580(10)$ & $16.3803(3)$ \\
\hline $\mathrm{b}(\AA)$ & $19.9207(2)$ & $29.3997(6)$ \\
\hline c $(\AA)$ & $24.04610(10)$ & $34.6846(5)$ \\
\hline$\alpha(\operatorname{deg})$ & $85.6310(10)$ & $70.914(2)$ \\
\hline$\beta(\operatorname{deg})$ & $83.5720(10)$ & $88.7450(10)$ \\
\hline$\gamma(\operatorname{deg})$ & $87.6860(10)$ & $88.2070(10)$ \\
\hline $\mathrm{V}\left(\AA^{3}\right)$ & $3721.85(6)$ & $15776.1(5)$ \\
\hline $\mathrm{Z}$ & 2 & 2 \\
\hline $\mathrm{D}_{\text {calc }}\left(\mathrm{g} / \mathrm{cm}^{3}\right)$ & 1.203 & 1.135 \\
\hline$\mu\left(\mathrm{mm}^{-1}\right)$ & 0.560 & 0.529 \\
\hline $\mathrm{F}(000)$ & 1424.0 & 5696.0 \\
\hline Crystal size $\left(\mathrm{mm}^{3}\right)$ & $0.260 \times 0.250 \times 0.050$ & $0.400 \times 0.380 \times 0.330$ \\
\hline Radiation & $\operatorname{CuK} \alpha(\lambda=1.54184)$ & $\mathrm{CuK} \alpha(\lambda=1.54184)$ \\
\hline$\Theta$ range $(\mathrm{deg})$ & $2.23-66.50$ & $2.69-66.05$ \\
\hline Reflns collected & 130280 & 166904 \\
\hline Indep reflns / $\mathrm{R}_{\text {int }}$ & $13037 / 0.0385$ & $53748 / 0.0797$ \\
\hline Data/restraints/parameters & $13037 / 145 / 937$ & $53748 / 1196 / 3741$ \\
\hline GOF on $F^{2}$ & 1.890 & 1.782 \\
\hline $\mathrm{R}_{1}, \mathrm{w} R_{2}[\mathrm{I}>=2 \sigma(\mathrm{I})]$ & $0.1106 / 0.3784$ & $0.1979 / 0.4492$ \\
\hline $\mathrm{R}_{1}, \mathrm{w} R_{2}$ [all data] & $0.1170 / 0.3952$ & $0.2294 / 0.4780$ \\
\hline
\end{tabular}


Table S6. Crystal data and structure refinement for $(P, P)-\mathbf{P D 8 H - 6 R}$ and $(M, M)$-PD8H-6R

\begin{tabular}{|c|c|c|}
\hline Compound & $(P, P)$-PD8H-6R & $(M, M)$-PD8H-6R \\
\hline Empirical formula & $\mathrm{C}_{102} \mathrm{H}_{82} \mathrm{Cl}_{12} \mathrm{~N}_{2} \mathrm{O}_{4}$ & $\mathrm{C}_{102} \mathrm{H}_{82} \mathrm{Cl}_{12} \mathrm{~N}_{2} \mathrm{O}_{4}$ \\
\hline Formula weight & 1825.09 & 1825.09 \\
\hline $\mathrm{T}(\mathrm{K})$ & $169.99(12)$ & $169.99(11)$ \\
\hline Crystal system & monoclinic & monoclinic \\
\hline Space group & $\mathrm{P} 2_{1}$ & $\mathrm{P} 2_{1}$ \\
\hline $\mathrm{a}(\AA)$ & $15.2028(3)$ & $15.2129(4)$ \\
\hline $\mathrm{b}(\AA)$ & $14.7948(2)$ & $14.7868(4)$ \\
\hline$c(\AA)$ & $20.7050(7)$ & $20.7684(7)$ \\
\hline$\alpha(\operatorname{deg})$ & 90 & 90 \\
\hline$\beta(\operatorname{deg})$ & $104.281(3)$ & $103.878(3)$ \\
\hline$\gamma(\mathrm{deg})$ & 90 & 90 \\
\hline $\mathrm{V}\left(\AA^{3}\right)$ & $4513.1(2)$ & $4535.5(2)$ \\
\hline $\mathrm{Z}$ & 2 & 2 \\
\hline$D_{\text {calc }}\left(\mathrm{g} / \mathrm{cm}^{3}\right)$ & 1.343 & 1.336 \\
\hline$\mu\left(\mathrm{mm}^{-1}\right)$ & 3.796 & 3.778 \\
\hline $\mathrm{F}(000)$ & 1888.0 & 1888.0 \\
\hline Crystal size $\left(\mathrm{mm}^{3}\right)$ & $0.34 \times 0.33 \times 0.14$ & $0.34 \times 0.33 \times 0.14$ \\
\hline Radiation & $\mathrm{CuK} \alpha(\lambda=1.54184)$ & $\mathrm{CuK} \alpha(\lambda=1.54184)$ \\
\hline$\Theta$ range $(\mathrm{deg})$ & $3.00-76.29$ & $2.94-76.29$ \\
\hline Reflns collected & 161649 & 88080 \\
\hline Indep reflns / $R_{\text {int }}$ & $17768 / 0.1905$ & $17304 / 0.1112$ \\
\hline Data/restraints/parameters & $17768 / 468 / 1082$ & $17304 / 462 / 1081$ \\
\hline GOF on $F^{2}$ & 2.102 & 2.337 \\
\hline $\mathrm{R}_{1}, w R_{2}[\mathrm{I}>=2 \sigma(\mathrm{I})]$ & $0.1532 / 0.3610$ & $0.1375 / 0.3298$ \\
\hline $\mathrm{R}_{1}, \mathrm{w} R_{2}$ [all data] & 0.2094 / 0.3895 & $0.1559 / 0.3437$ \\
\hline Flack parameter & $0.12(3)$ & $0.148(14)$ \\
\hline
\end{tabular}


Table S7. Crystal data and structure refinement for PD8H-7R and PD8H-7M

\begin{tabular}{|c|c|c|}
\hline Compound & PD8H-7R & PD8H-7M \\
\hline Empirical formula & $\mathrm{C}_{98} \mathrm{H}_{78} \mathrm{~N}_{2} \mathrm{O}_{4}$ & $\mathrm{C}_{99} \mathrm{H}_{79} \mathrm{Cl}_{3} \mathrm{~N}_{2} \mathrm{O}_{4}$ \\
\hline Formula weight & 1347.62 & 1466.99 \\
\hline $\mathrm{T}(\mathrm{K})$ & $169.99(13)$ & $169.98(10)$ \\
\hline Crystal system & triclinic & triclinic \\
\hline Space group & $\mathrm{P}-1$ & $\mathrm{P}-1$ \\
\hline $\mathrm{a}(\AA)$ & $13.8600(3)$ & $7.71580(10)$ \\
\hline$b(\AA)$ & $17.3797(5)$ & $15.2504(3)$ \\
\hline$c(\AA)$ & $35.4965(5)$ & $16.7377(3)$ \\
\hline$\alpha(\operatorname{deg})$ & $83.053(2)$ & $69.440(2)$ \\
\hline$\beta(\operatorname{deg})$ & $83.981(2)$ & $82.721(2)$ \\
\hline$\gamma(\operatorname{deg})$ & $77.853(2)$ & $80.7830(10)$ \\
\hline $\mathrm{V}\left(\AA^{3}\right)$ & $8269.9(3)$ & $1814.96(6)$ \\
\hline $\mathrm{Z}$ & 4 & 1 \\
\hline$D_{\text {calc }}\left(\mathrm{g} / \mathrm{cm}^{3}\right)$ & 1.082 & 1.342 \\
\hline$\mu\left(\mathrm{mm}^{-1}\right)$ & 0.504 & 1.611 \\
\hline $\mathrm{F}(000)$ & 2848.0 & 770.0 \\
\hline Crystal size $\left(\mathrm{mm}^{3}\right)$ & $0.414 \times 0.021 \times 0.021$ & $0.62 \times 0.23 \times 0.05$ \\
\hline Radiation & $\mathrm{CuK} \alpha(\lambda=1.54178)$ & $\mathrm{CuK} \alpha(\lambda=1.54184)$ \\
\hline$\Theta$ range $(\mathrm{deg})$ & 2.51-66.99 & $2.82-75.08$ \\
\hline Reflns collected & 287682 & 64481 \\
\hline Indep reflns / $R_{\text {int }}$ & $28806 / 0.1617$ & $7216 / 0.0576$ \\
\hline Data/restraints/parameters & $28806 / 1067 / 2003$ & $7216 / 309 / 572$ \\
\hline GOF on $F^{2}$ & 1.291 & 1.050 \\
\hline $\mathrm{R}_{1}, \mathrm{w} R_{2}[\mathrm{I}>=2 \sigma(\mathrm{I})]$ & $0.1651 / 0.4012$ & $0.0738 / 0.2179$ \\
\hline $\mathrm{R}_{1}, \mathrm{w} R_{2}$ [all data] & $0.2147 / 0.4326$ & $0.0777 / 0.2227$ \\
\hline
\end{tabular}


a)

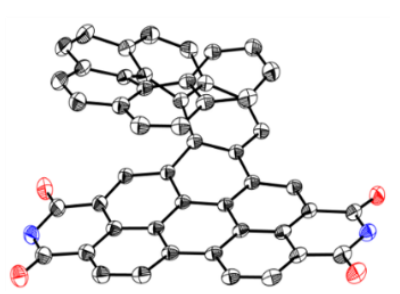

b)

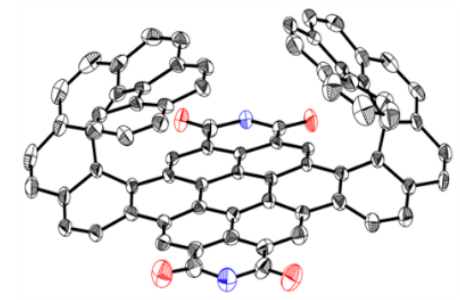

d)

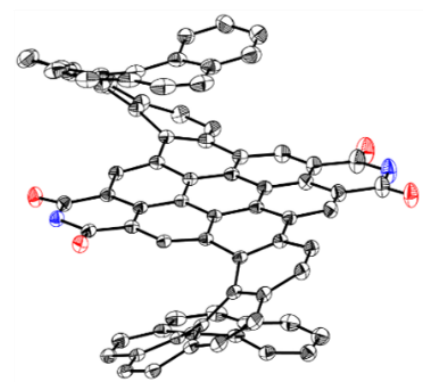

c)

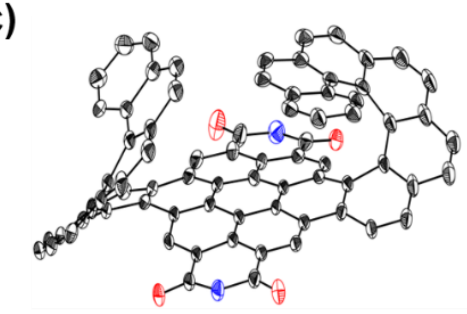

e)

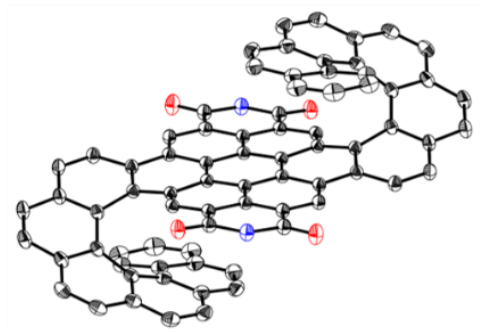

Figure S13. Single crystals of $P$-P8H (a), $(M, P)$-PD8H-6M (b), $(P, P)$-PD8H-7R (c), $(P, P)$-PD8H-6R (d), and $(M, P)$-PD8H-7M (e).
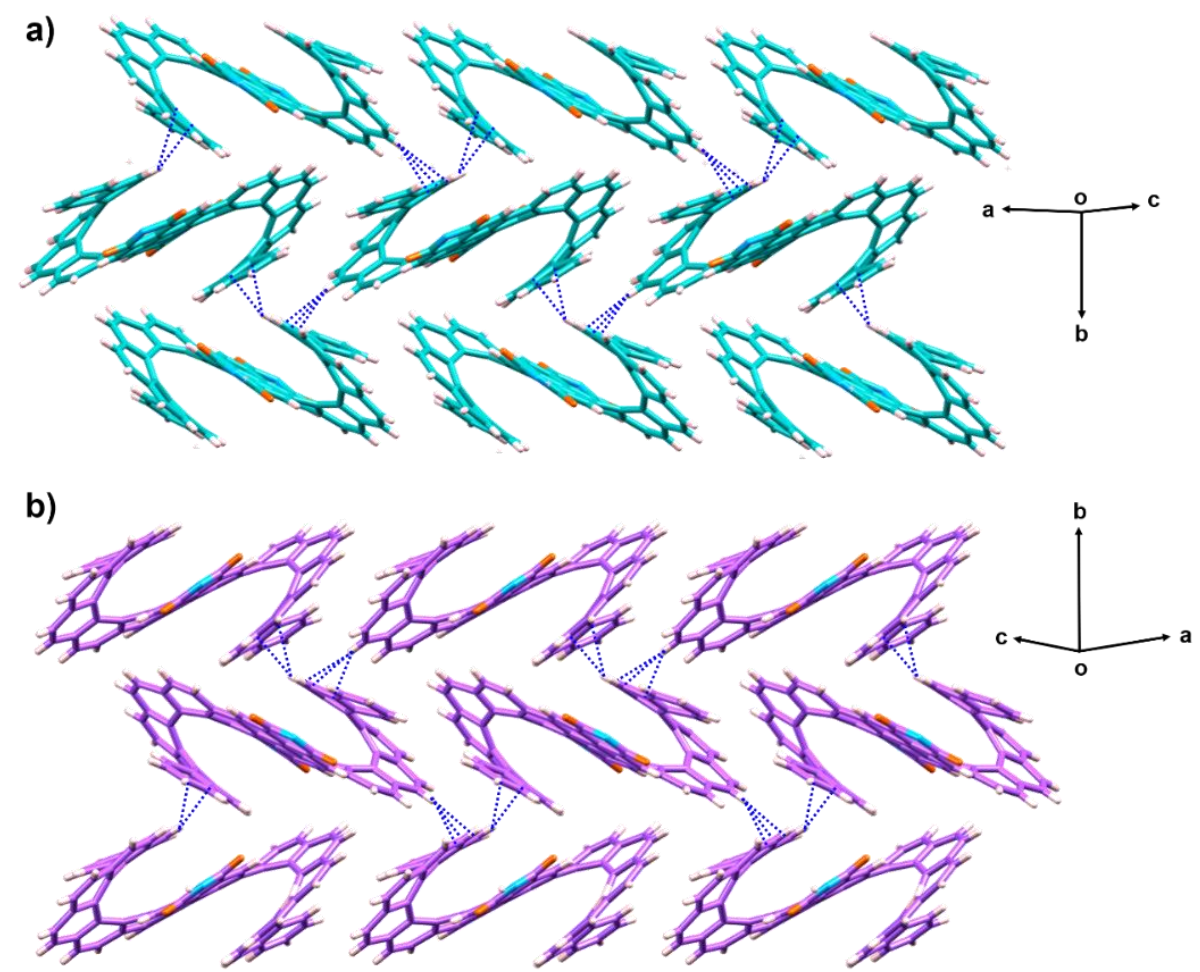

Figure S14. Molecular packing diagram of enantiomerically pure $(M, M)$-PD8H-6R (a) and $(P$, P)-PD8H-6R (b). The blue dotted lines represent the intermolecular C-H $\cdots \pi$ contacts. 


\section{Theoretical Calculations}

The density functional theory (DFT) calculations were performed with the Gaussian 09 Rev. D.01 quantum chemistry package ${ }^{[4]}$ employing the B3LYP ${ }^{[5]}$ exchange-correlation functional and the $6-31 \mathrm{G}^{*}$ basis set. The long alkyl chains of the chemical compounds were reduced to methyl groups and all calculations took into account the solvent used during experiments (chloroform) through the self-consistent reaction field (SCRF) method using the polarizable continuum model $(\mathrm{PCM})^{[6]}$ and the UFF atomic radii model (i.e., explicit hydrogens). The optimized structures and transition state (TS) where confirmed by harmonic frequency calculations.

The UV-Vis absorption and electronic circular dichroism $(\mathrm{ECD})^{[7]}$ spectra were calculated using the time-dependent DFT (TDDFT) linear response method. For each molecule, the lowest 100 states were computed at the optimized geometry. The calculated line spectra are represented by applying Lorentz broadening with a full width at half maximum (FWHM) of $50 \mathrm{~nm}$.

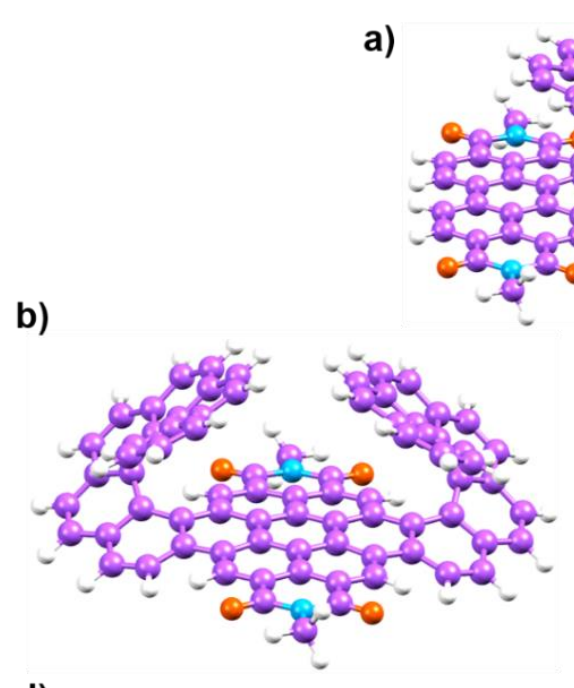

d)

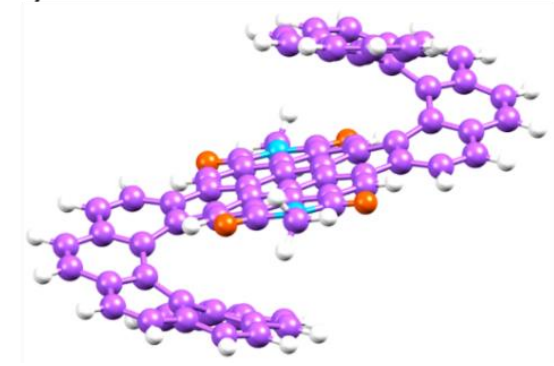

c)

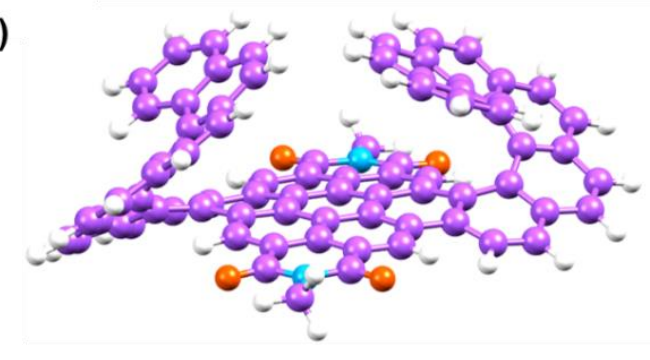

e)

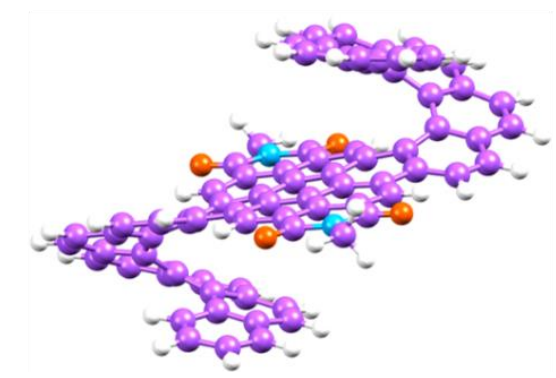

Figure S15. Visualisation of the optimized structures of $P$-P8H (a), $(M, P)-\mathbf{P D 8 H - 6 M}$ (b), $(P, P)$-PD8H-7R (c), $(P, P)$-PD8H-6R (d), and $(M, P)-$ PD8H-7M (e). 


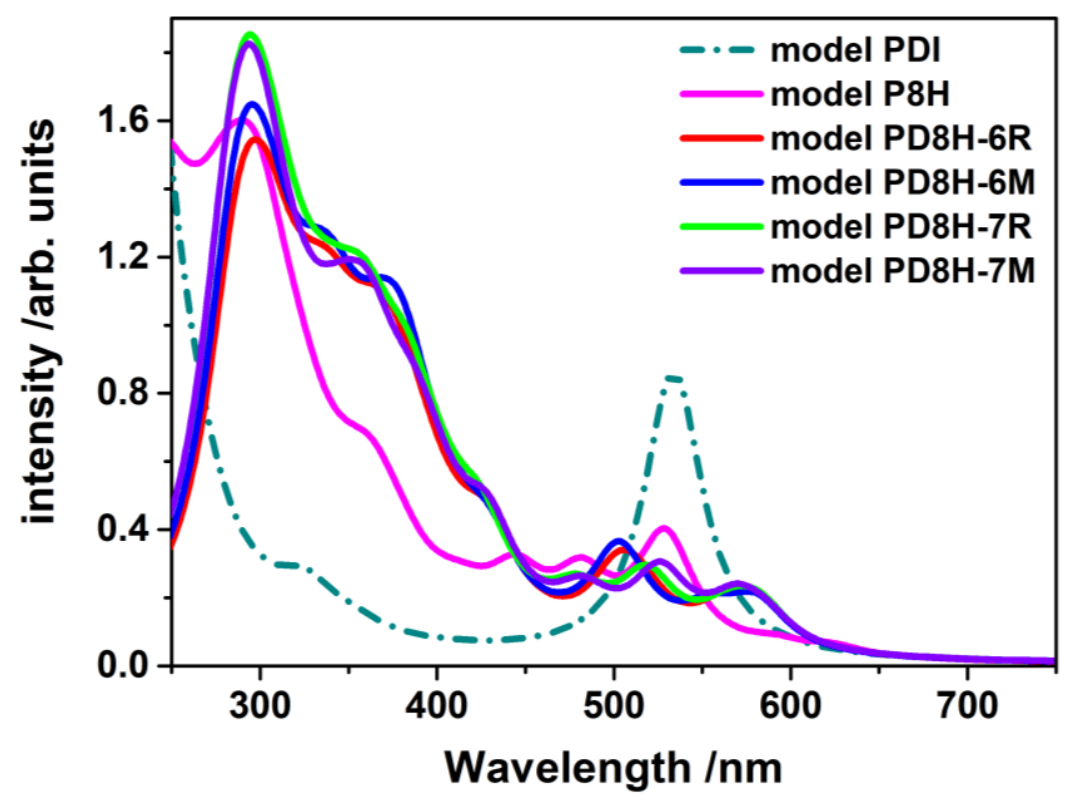

Figure S16. Theoretical UV/vis absorption curves from B3LYP/6-31G* TDDFT line spectrum calculations and Lorentzian line shape with line broadening of $40 \mathrm{~nm}$ (FWHM).
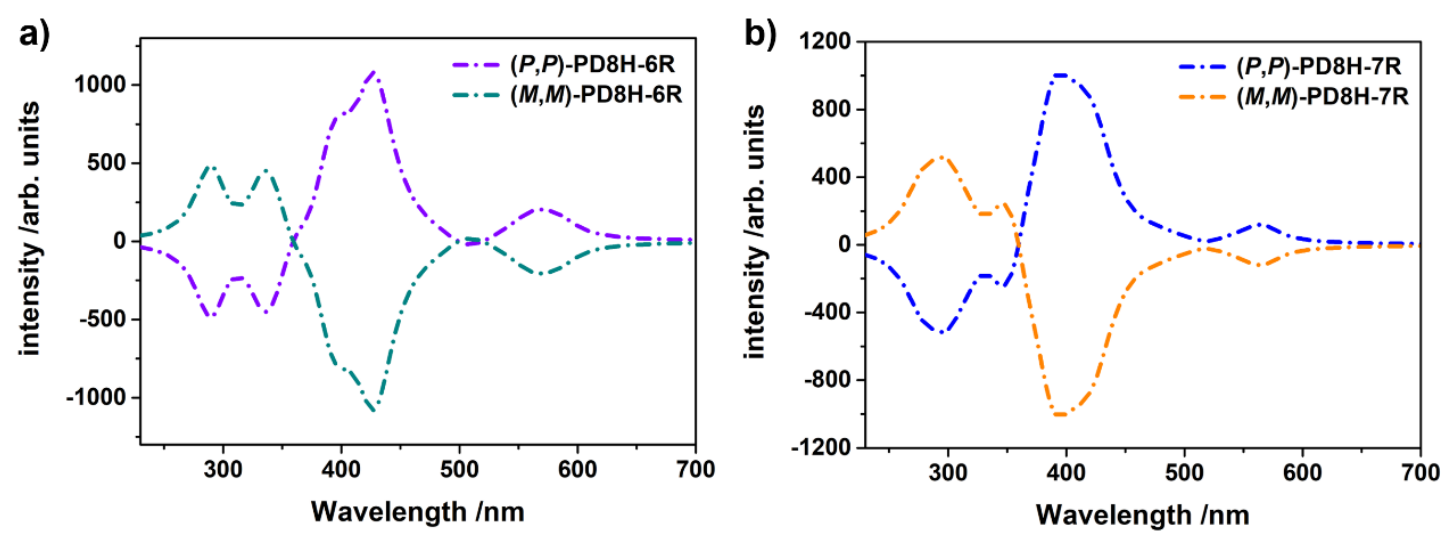

Figure S17. Theoretical ECD absorption curves for PD8H-6R and PD8H-7R from B3LYP/6-31G* TDDFT line spectrum calculations and Lorentzian line shape with line broadening of $40 \mathrm{~nm}$ (FWHM).

Table S8. TDDFT excitation energies, oscillator strengths, and leading WF contributions for PDI

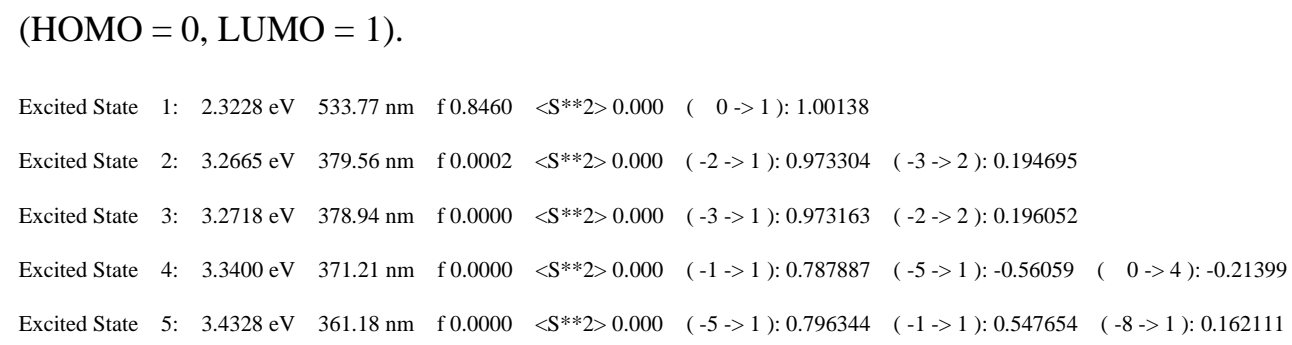




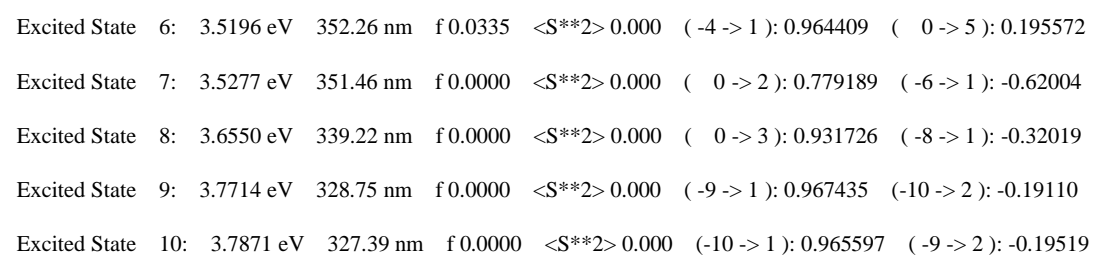

Table S9. TDDFT excitation energies, oscillator strengths, and leading WF contributions for P8H $(\mathrm{HOMO}=0, \mathrm{LUMO}=1)$.

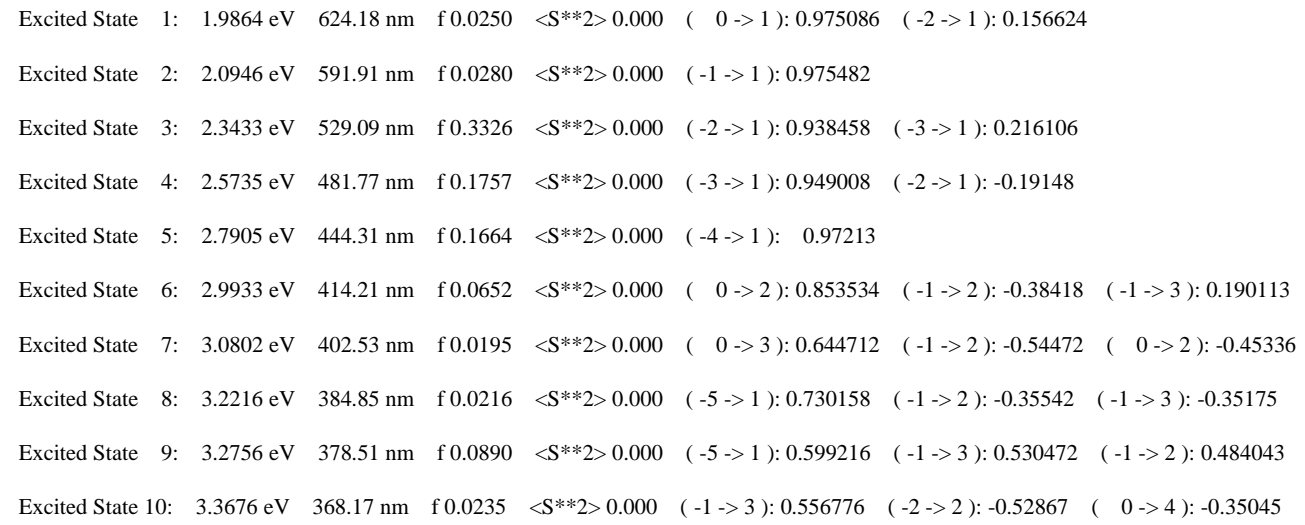

Table S10. TDDFT excitation energies, oscillator strengths, and leading WF contributions for PD8H-6R $\quad(\mathrm{HOMO}=0, \mathrm{LUMO}=1)$.

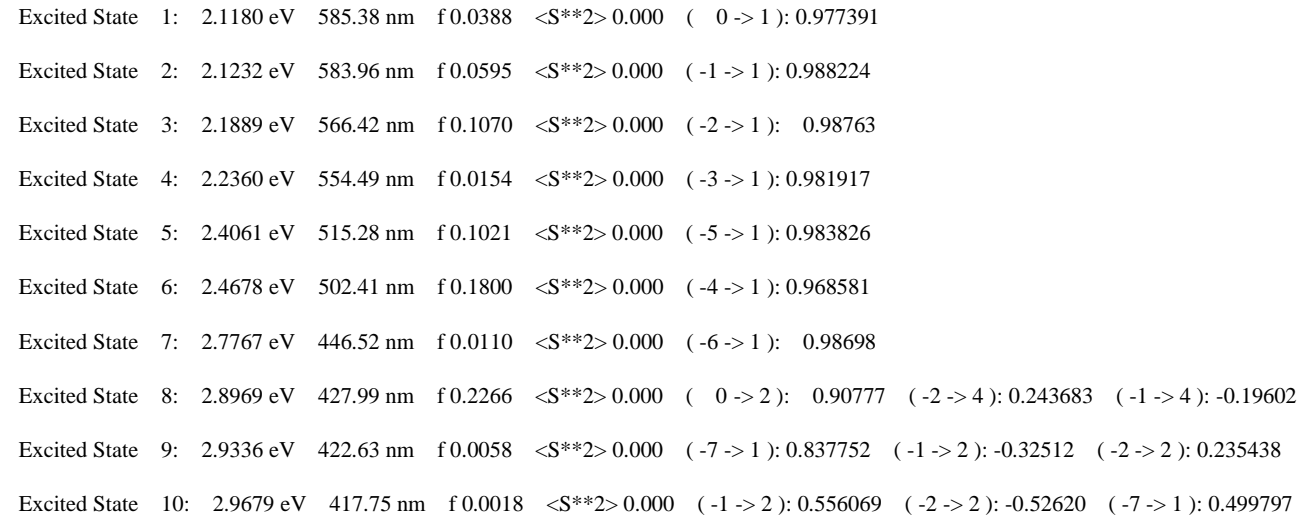

Table S11. TDDFT excitation energies, oscillator strengths, and leading WF contributions for PD8H-6M $\quad(\mathrm{HOMO}=0, \mathrm{LUMO}=1)$.

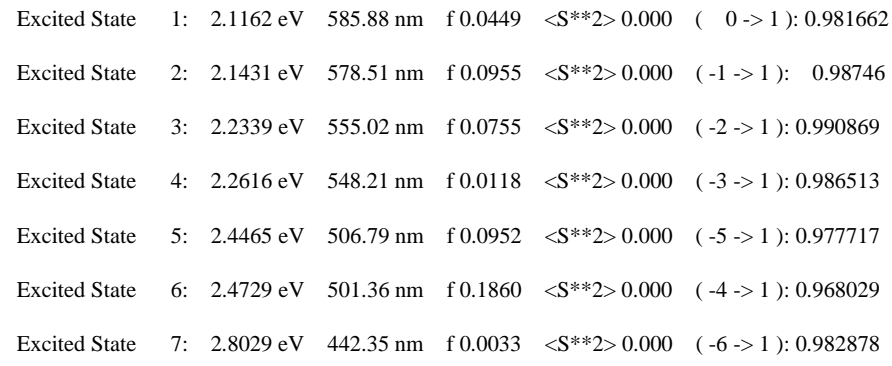




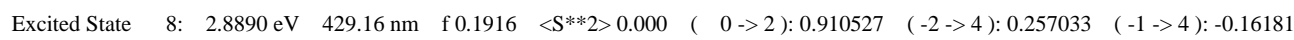

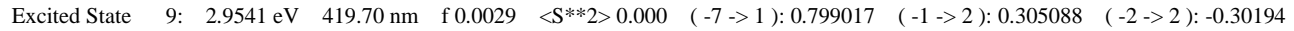

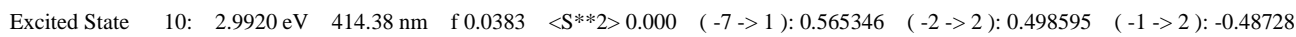

Table S12. TDDFT excitation energies, oscillator strengths, and leading WF contributions for

PD8H-7R $(\mathrm{HOMO}=0, \mathrm{LUMO}=1)$.

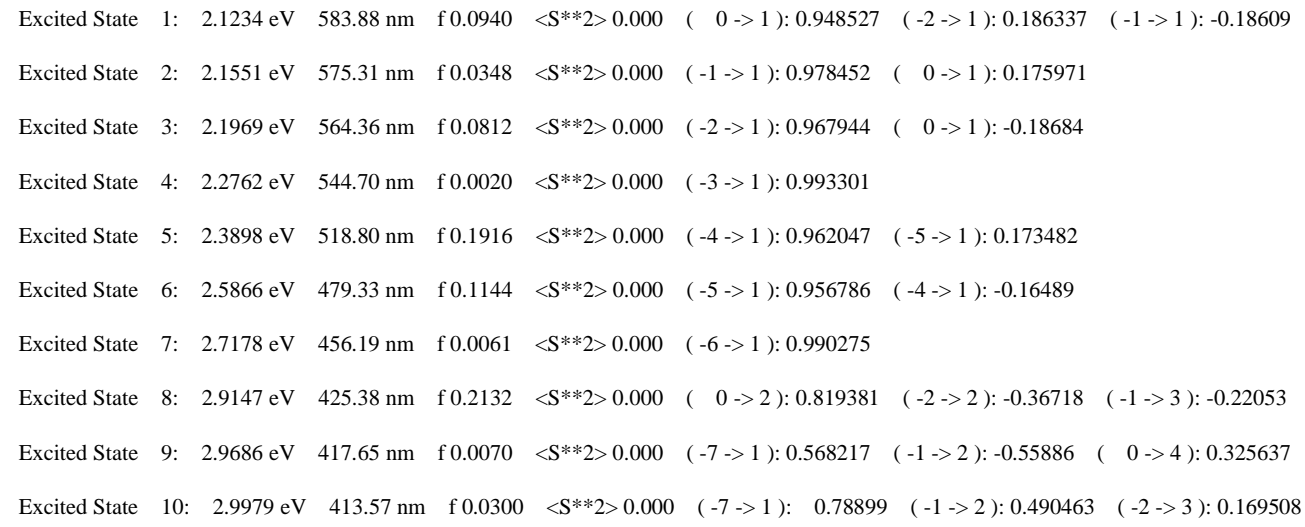

Table S13. TDDFT excitation energies, oscillator strengths, and leading WF contributions for

PD8H-7M $(\mathrm{HOMO}=0, \mathrm{LUMO}=1)$.

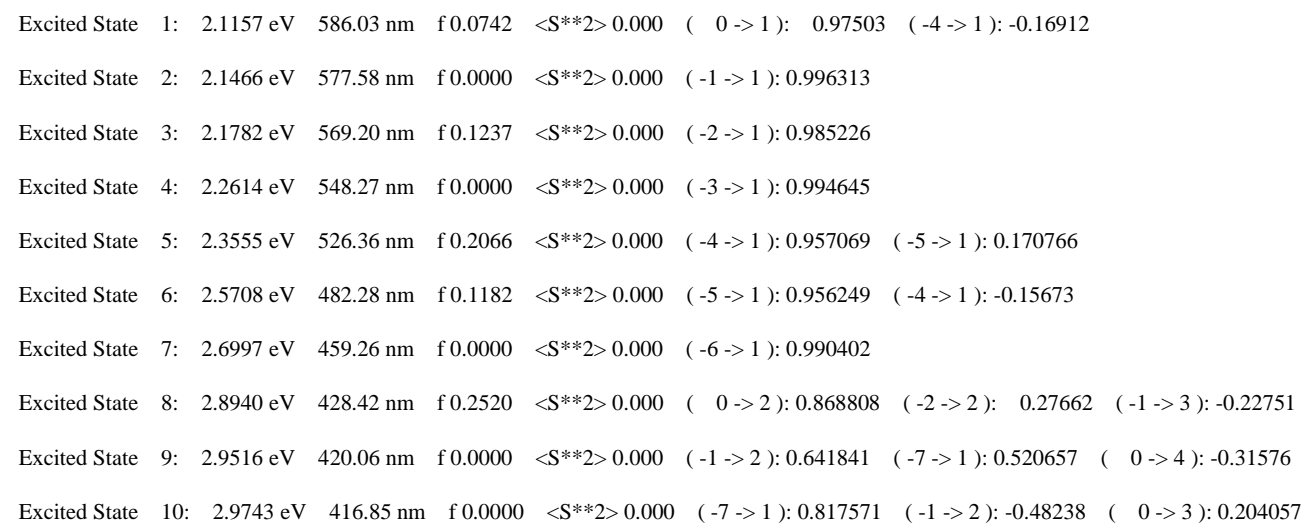

Table S14. Frontier Molecular Orbital Energies (eV)

\begin{tabular}{ccc}
\hline & $\begin{array}{c}\boldsymbol{E}_{\text {LUMO }} \\
{[\mathbf{e V}]}\end{array}$ & $\begin{array}{c}\boldsymbol{E}_{\text {HOMO }} \\
{[\mathrm{eV}]}\end{array}$ \\
\hline PDI & -3.38 & -5.90 \\
P8H & -3.15 & -5.60 \\
PD8H-6R & -2.98 & -5.54 \\
PD8H-6M & -2.97 & -5.52 \\
PD8H-7R & -2.97 & -5.54 \\
PD8H-7M & -2.97 & -5.53 \\
\hline
\end{tabular}




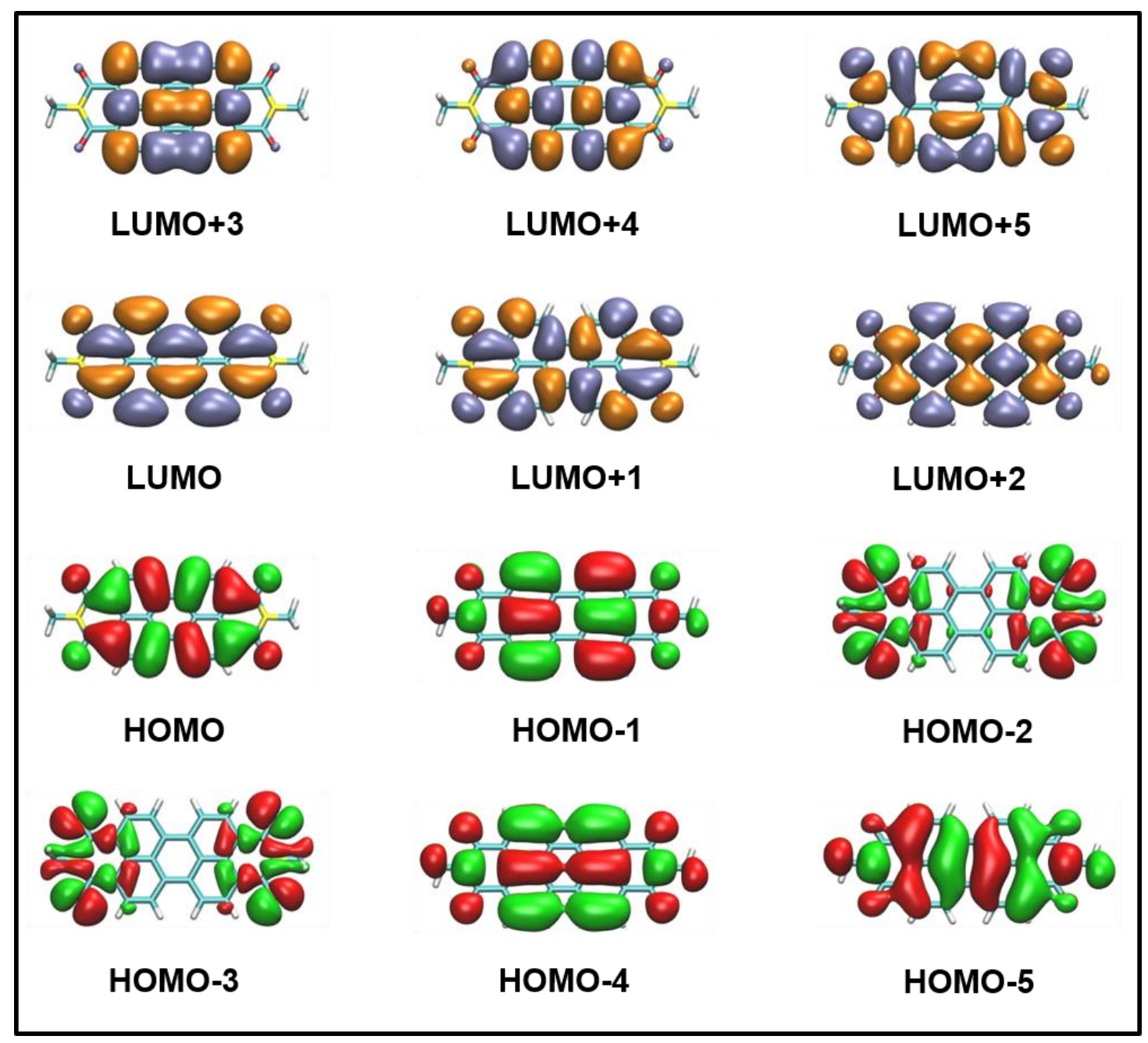

Figure S18. View of molecular orbitals of PDI. 


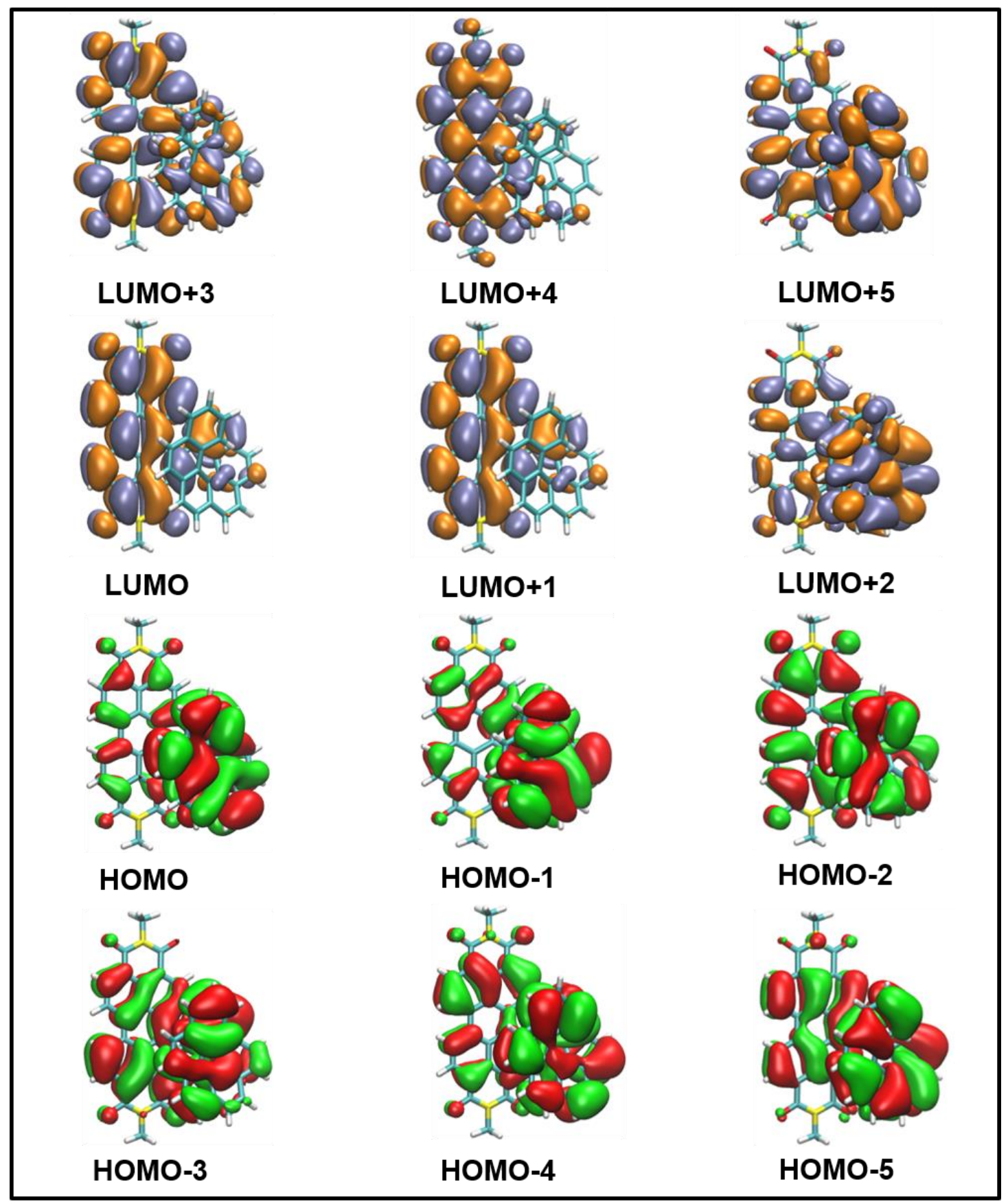

Figure S19. View of molecular orbitals of P8H. 


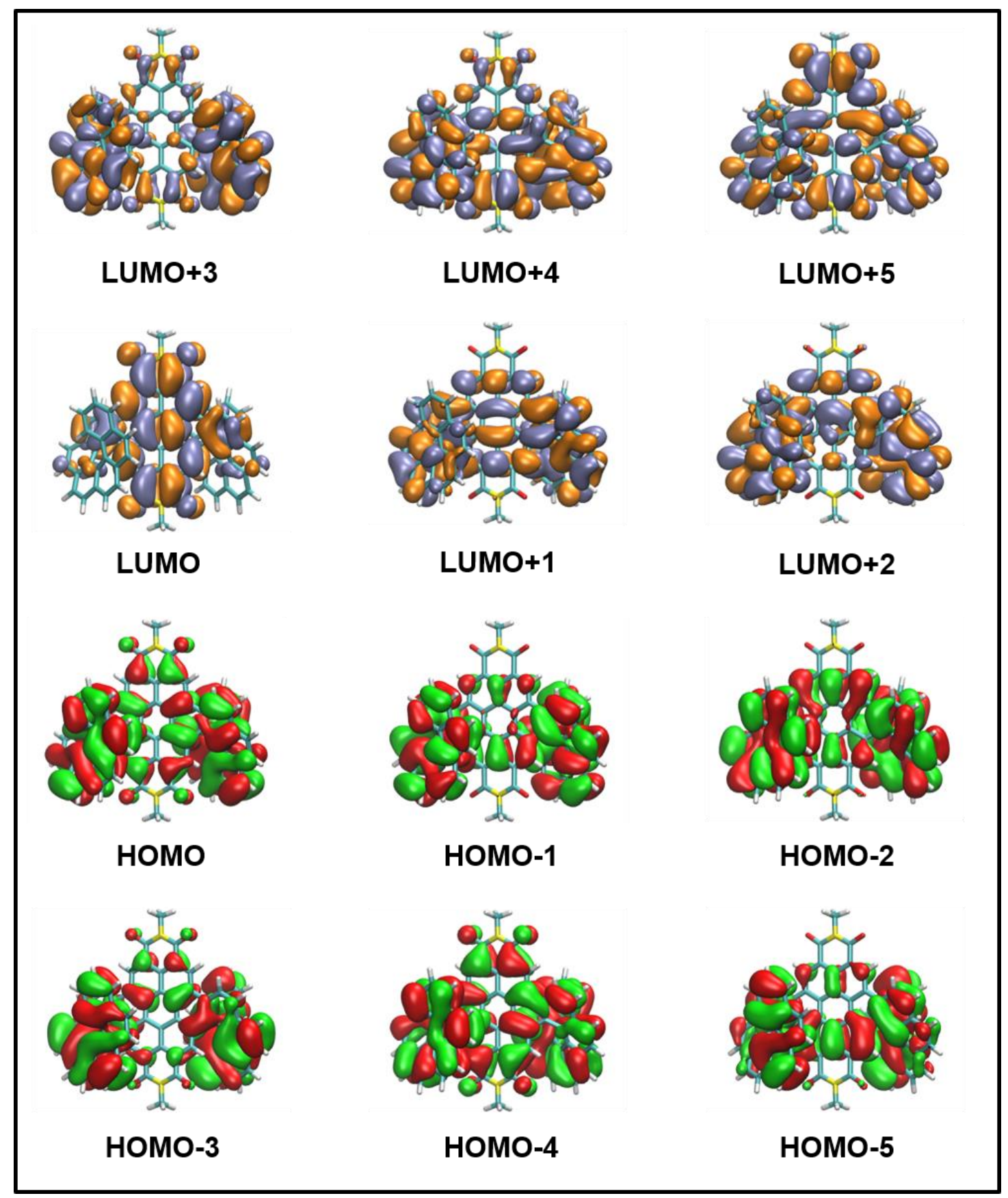

Figure S20. View of molecular orbitals of PD8H-6R. 


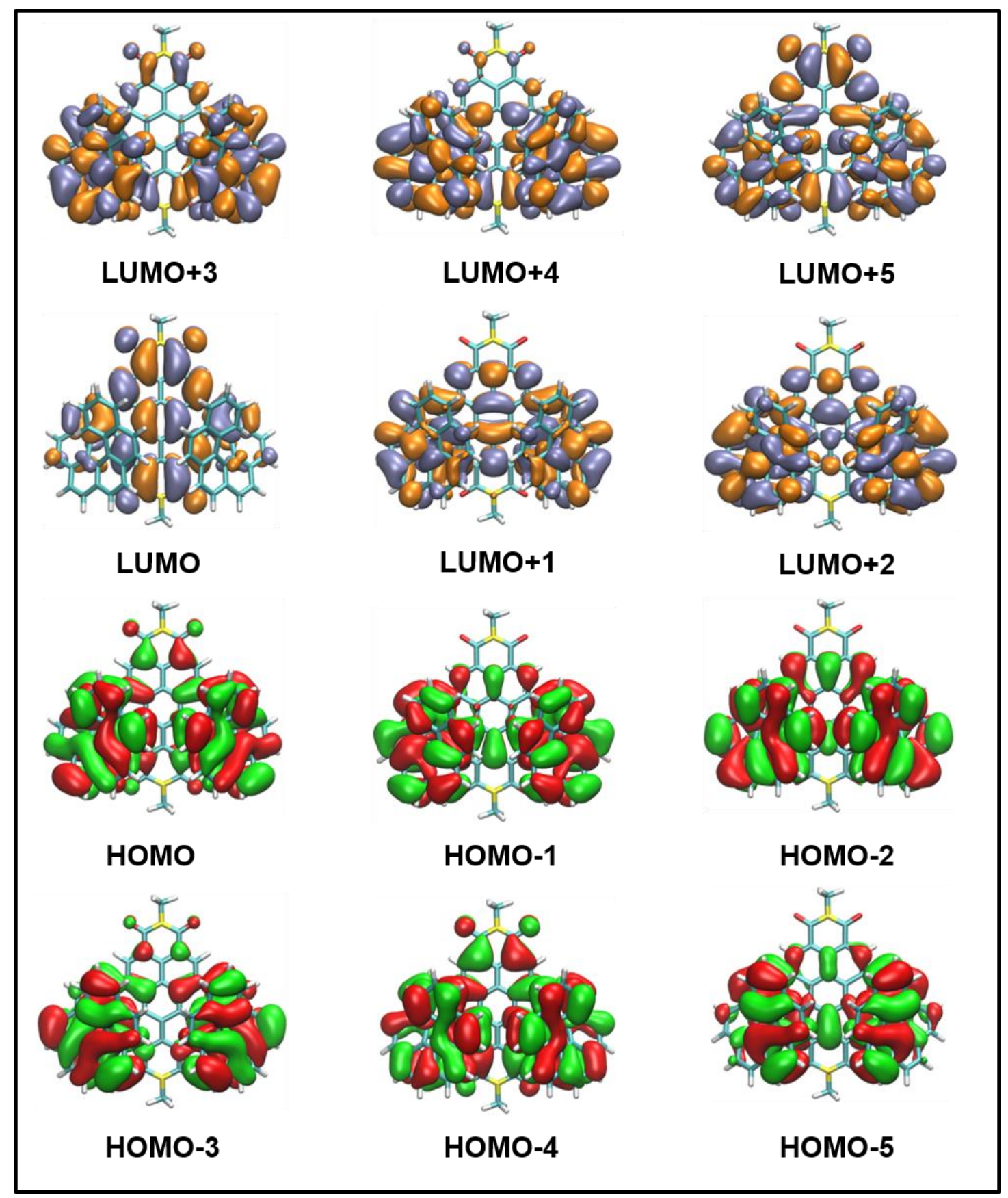

Figure S21. View of molecular orbitals of PD8H-6M. 


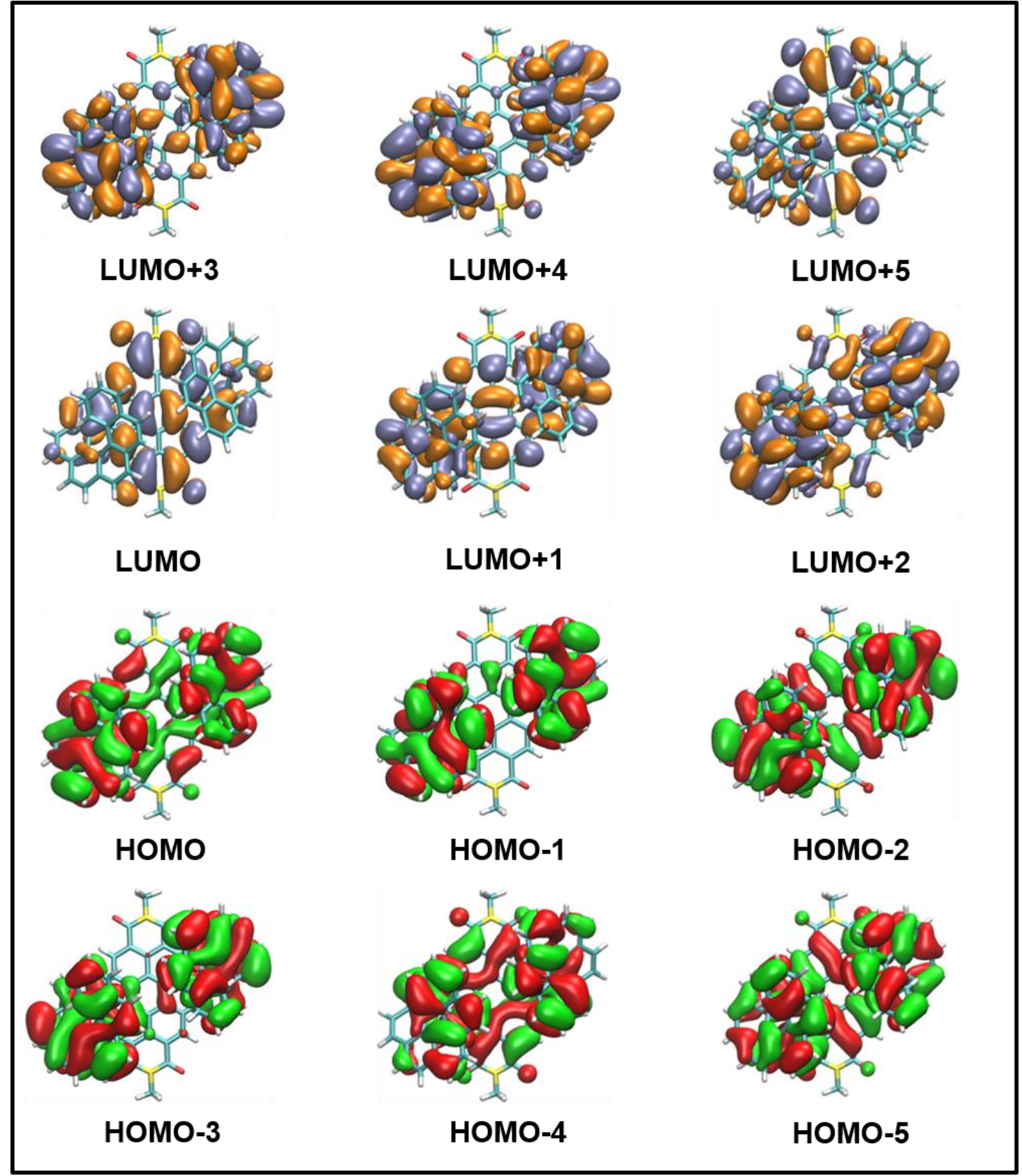

Figure S22. View of molecular orbitals of PD8H-7R. 


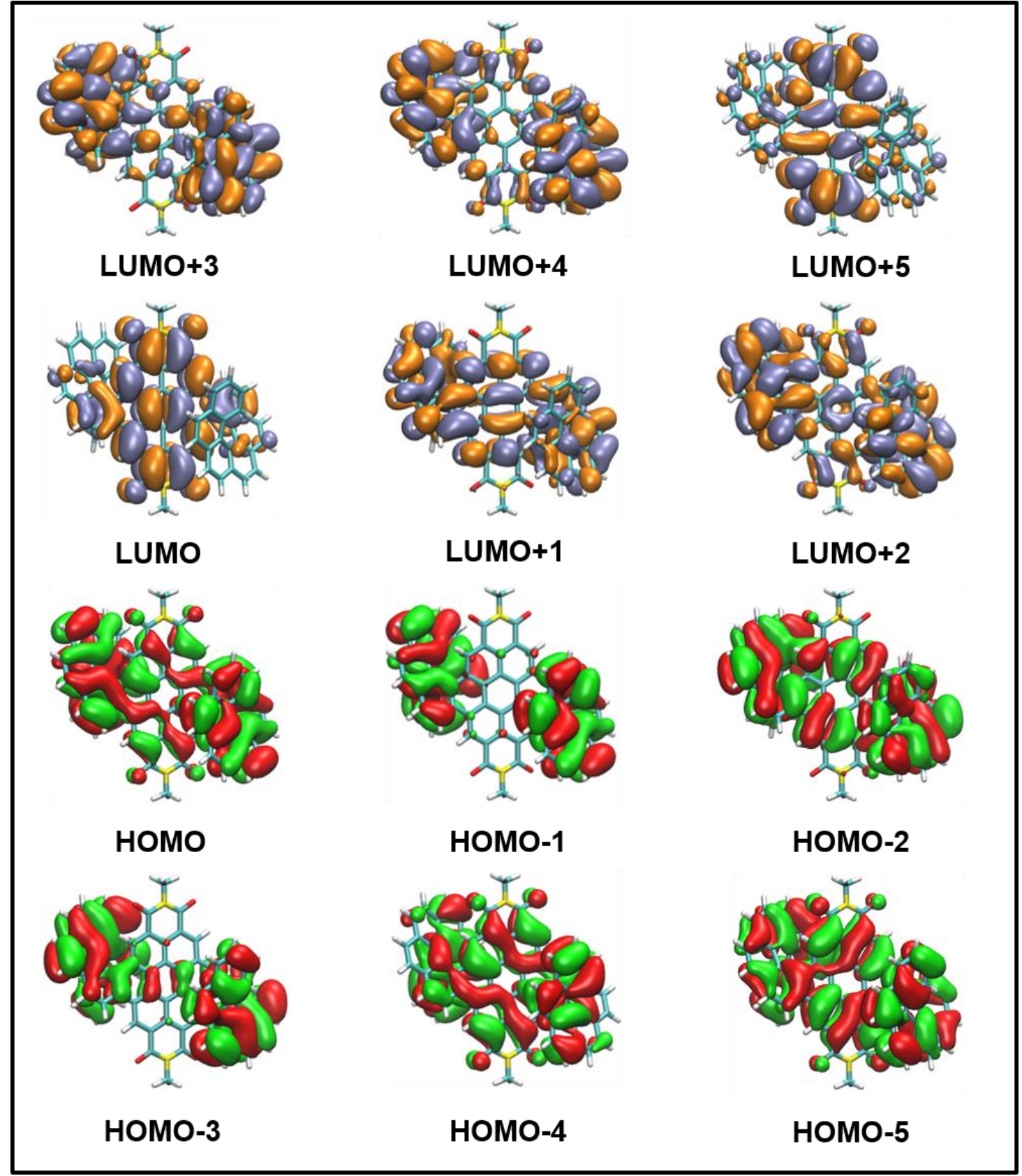

Figure S23. View of molecular orbitals of PD8H-7M. 


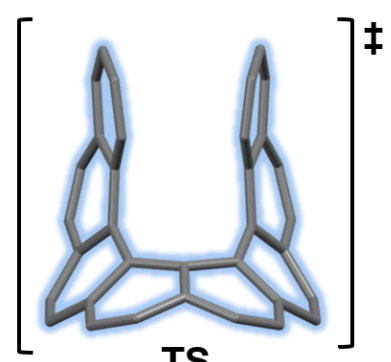

TS

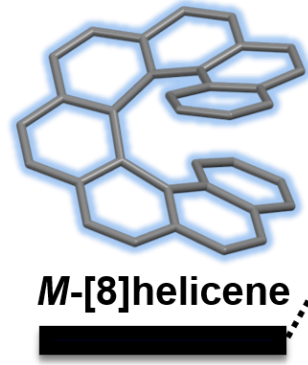

0.0

42.0

Figure S24. Activation barrier for the isomerization process between $M$-[8]helicene and $P$-[8]helicene in units of $\mathrm{kcal} / \mathrm{mol}$. The relative Gibbs free energy was calculated at the B3LYP/6-31G* level of theory.

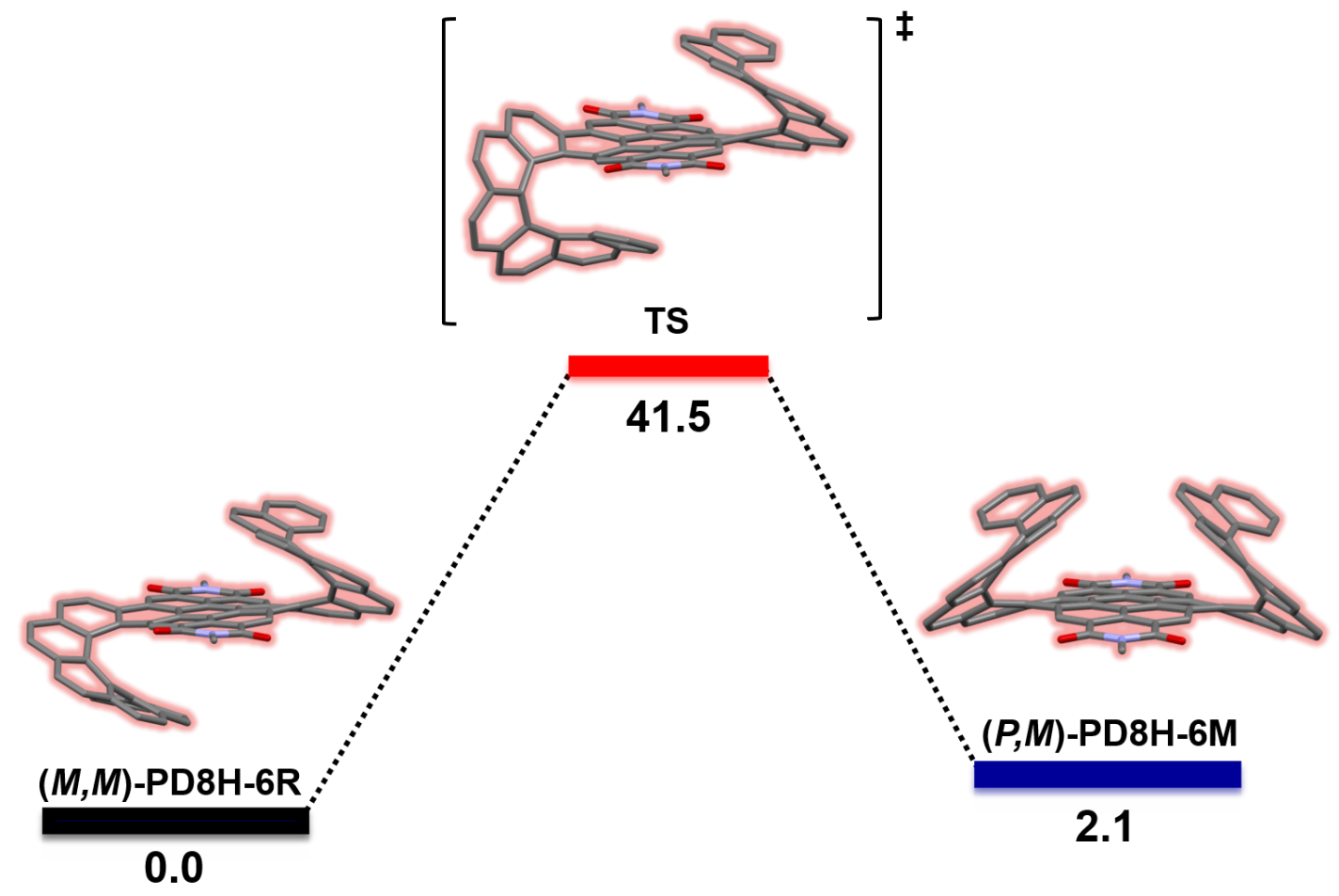

Figure S25. Activation barrier for the isomerization process between $(M, M)$-PD8H-6R and $(P, M)$-PD8H-6M in units of $\mathrm{kcal} / \mathrm{mol}$. The relative Gibbs free energy was calculated at the B3LYP/6-31G* level of theory. 
Table S15. The cartesian coordinates of some optimized structures are listed as follows:

\begin{tabular}{|c|c|c|c|}
\hline \multicolumn{4}{|c|}{$M$-[8]helicene } \\
\hline $\mathrm{C}$ & 0.69511200 & -2.05844800 & 2.86200000 \\
\hline $\mathrm{C}$ & 0.04263500 & -1.03004100 & 2.21101000 \\
\hline $\mathrm{C}$ & 0.73649900 & 0.11376600 & 1.73968300 \\
\hline $\mathrm{C}$ & 2.12731700 & 0.21028800 & 2.06079500 \\
\hline $\mathrm{C}$ & 2.08599700 & -2.00041400 & 3.07897100 \\
\hline $\mathrm{C}$ & 2.78200500 & -0.87462000 & 2.69360000 \\
\hline $\mathrm{C}$ & 0.07827500 & 1.23116400 & 1.07465000 \\
\hline $\mathrm{C}$ & 0.75981900 & 2.47809900 & 1.07569000 \\
\hline $\mathrm{C}$ & 2.15007400 & 2.54043800 & 1.40998600 \\
\hline $\mathrm{C}$ & 2.82674300 & 1.43429500 & 1.82310500 \\
\hline $\mathrm{C}$ & -1.25917000 & 1.18836500 & 0.51111300 \\
\hline $\mathrm{C}$ & -1.98169800 & 2.41714000 & 0.44567800 \\
\hline $\mathrm{C}$ & -1.29427700 & 3.65786600 & 0.58090200 \\
\hline $\mathrm{C}$ & 0.05442600 & 3.68218600 & 0.78759600 \\
\hline $\mathrm{C}$ & -1.92802900 & -0.00064300 & -0.00024400 \\
\hline $\mathrm{C}$ & -3.35381900 & -0.00118600 & -0.00025200 \\
\hline $\mathrm{C}$ & -4.06818000 & 1.22658800 & 0.13120400 \\
\hline $\mathrm{C}$ & -3.39422500 & 2.40693100 & 0.24454700 \\
\hline $\mathrm{C}$ & -1.25816700 & -1.18911300 & -0.51137500 \\
\hline $\mathrm{C}$ & -1.97977900 & -2.41847700 & -0.44583800 \\
\hline $\mathrm{C}$ & -3.39231700 & -2.40931900 & -0.24487800 \\
\hline $\mathrm{C}$ & -4.06724500 & -1.22946200 & -0.13170100 \\
\hline $\mathrm{C}$ & 0.07940300 & -1.23099800 & -1.07463100 \\
\hline $\mathrm{C}$ & 0.76195000 & -2.47749700 & -1.07532800 \\
\hline $\mathrm{C}$ & 0.05741400 & -3.68202700 & -0.78711500 \\
\hline $\mathrm{C}$ & -1.29137400 & -3.65868400 & -0.58071100 \\
\hline $\mathrm{C}$ & 0.73683600 & -0.11334500 & -1.73978700 \\
\hline $\mathrm{C}$ & 2.12792000 & -0.20884000 & -2.06061900 \\
\hline $\mathrm{C}$ & 2.82816100 & -1.43226700 & -1.82260800 \\
\hline $\mathrm{C}$ & 2.15218100 & -2.53882600 & -1.40940600 \\
\hline $\mathrm{C}$ & 0.04228500 & 1.02985100 & -2.21163900 \\
\hline $\mathrm{C}$ & 0.69418200 & 2.05872900 & -2.86242600 \\
\hline $\mathrm{C}$ & 2.08521300 & 2.00183700 & -3.07886700 \\
\hline $\mathrm{C}$ & 2.78191100 & 0.87655800 & -2.69322500 \\
\hline $\mathrm{H}$ & 2.59716200 & -2.82012800 & 3.57671500 \\
\hline $\mathrm{H}$ & 3.84572600 & -0.78558100 & 2.90233300 \\
\hline $\mathrm{H}$ & 3.88814500 & 1.48347900 & 2.05371100 \\
\hline $\mathrm{H}$ & 2.65482900 & 3.49949800 & 1.32271700 \\
\hline $\mathrm{H}$ & 0.59791900 & 4.62333000 & 0.81535000 \\
\hline $\mathrm{H}$ & -1.86112500 & 4.57963300 & 0.47557400 \\
\hline $\mathrm{H}$ & -3.92458400 & 3.35596700 & 0.24295500 \\
\hline $\mathrm{H}$ & -5.15389300 & 1.20369500 & 0.08148100 \\
\hline
\end{tabular}




$\begin{array}{lrrr}\mathrm{H} & -5.15297800 & -1.20745700 & -0.08206500 \\ \mathrm{H} & -3.92197100 & -3.35874700 & -0.24320400 \\ \mathrm{H} & -1.85745900 & -4.58089800 & -0.47521600 \\ \mathrm{H} & 0.60157800 & -4.62279500 & -0.81453700 \\ \mathrm{H} & 2.65765300 & -3.49750600 & -1.32194900 \\ \mathrm{H} & 3.88958700 & -1.48085900 & -2.05319000 \\ \mathrm{H} & 3.84575900 & 0.78825800 & -2.90169300 \\ \mathrm{H} & 2.59591000 & 2.82194000 & -3.57642500 \\ \mathrm{H} & 0.12445200 & 2.91344100 & -3.21684600 \\ \mathrm{H} & -1.03081700 & 1.08768200 & -2.08641400 \\ \mathrm{H} & 0.12597700 & -2.91363300 & 3.21621700 \\ \mathrm{H} & -1.03034200 & -1.08869100 & 2.08520400\end{array}$

\section{$P$-[8]helicene}

C $\quad-0.04264200$

$-2.05860500$

2.86208200

C $\quad-0.73682500$

$-1.02994600$

2.21125500

C $\quad-2.12773500$

0.11350300

1.73958000

C $\quad-2.08587300$

0.20953500

2.06039500

$\begin{array}{ll}\text { C } & -2.78218100\end{array}$

$-2.00111600$

3.07866800

C $\quad-0.07888800$

$-0.87556200$

2.69311900

C $\quad-0.76090100$

1.23106800

1.07451700

C $\quad-2.15126500$

2.47777900

1.07539700

2.53960900

1.40937200

C $\quad-2.82759600$

1.43326500

1.82243200

C $\quad 1.25863200$

1.18870000

0.51136600

C $\quad 1.98077700$

2.41777100

0.44594200

C $\quad 1.29288400$

3.65821600

0.58092300

C $\quad-0.05589200$

3.68207400

0.78740900

C $\quad 1.92804200$

$-0.00011300$

0.00018500

C $\quad 3.35381500$

$-0.00015700$

0.00031400

C $\quad 4.06772900$
C

1.22785400

0.13196200

C $\quad 3.39334100$

2.40798700

0.24510600

C $\quad 1.25863100$

$-1.18879300$

$-0.51110400$

C $\quad 1.98070300$

$-2.41790400$

$-0.44589800$

C $\quad 3.39319700$

$-2.40827300$

$-0.24482200$

C $\quad 4.06765000$

$-1.22818300$

$-0.13132400$

C $\quad-0.07894800$

$-1.23102700$

$-1.07430500$

C $\quad-0.76100300$

$-2.47765600$

$-1.07538800$

C $\quad-0.05603400$

$-3.68207100$

$-0.78757500$

C $\quad 1.29270700$

$-3.65833200$

$-0.58109200$

C $\quad-0.73659200$

$-0.11342200$

$-1.73944900$

C $\quad-2.12745300$

$-0.20927200$

$-2.06052100$

C $\quad-2.82747900$

$-1.43290400$

$-1.82262700$

C $\quad-2.15131100$

$-2.53935600$

$-1.40950300$

C

$-0.04210500$

1.02982400

$-2.21117400$ 


$\begin{array}{rrrr}\mathrm{C} & -0.69405900 & 2.05846700 & -2.86233200 \\ \mathrm{C} & -2.08500400 & 2.00119400 & -3.07914300 \\ \mathrm{C} & -2.78160300 & 0.87586700 & -2.69350600 \\ \mathrm{H} & -2.59686100 & -2.82099200 & 3.57632900 \\ \mathrm{H} & -3.84598100 & -0.78684600 & 2.90159100 \\ \mathrm{H} & -3.88903400 & 1.48208900 & 2.05294000 \\ \mathrm{H} & -2.65634500 & 3.49847600 & 1.32181800 \\ \mathrm{H} & -0.59965600 & 4.62306600 & 0.81500000 \\ \mathrm{H} & 1.85935800 & 4.58022400 & 0.47564500 \\ \mathrm{H} & 3.92344500 & 3.35717100 & 0.24362900 \\ \mathrm{H} & 5.15346100 & 1.20540100 & 0.08243800 \\ \mathrm{H} & 5.15337500 & -1.20583600 & -0.08156800 \\ \mathrm{H} & 3.92326900 & -3.35747500 & -0.24347000 \\ \mathrm{H} & 1.85917000 & -4.58036100 & -0.47595700 \\ \mathrm{H} & -0.59987700 & -4.62301200 & -0.81548500 \\ \mathrm{H} & -2.65648200 & -3.49819500 & -1.32211100 \\ \mathrm{H} & -3.88889500 & -1.48161500 & -2.05324500 \\ \mathrm{H} & -3.84541300 & 0.78736000 & -2.90203200 \\ \mathrm{H} & -2.59574200 & 2.82106800 & -3.57706400 \\ \mathrm{H} & -0.12441700 & 2.91323700 & -3.21672400 \\ \mathrm{H} & 1.03091700 & 1.08796700 & -2.08538500 \\ \mathrm{H} & -0.12550500 & -2.91358300 & 3.21635100 \\ \mathrm{H} & 1.03039900 & -1.08826600 & 2.08573900\end{array}$

\section{TS of [8] helicene}

$\begin{array}{lrrr}\mathrm{C} & 0.24783400 & -0.10085300 & -0.04008600 \\ \mathrm{C} & 0.16152900 & -0.08354800 & 1.33802200 \\ \mathrm{C} & 1.32208700 & -0.03385800 & 2.14819400 \\ \mathrm{C} & 2.59389200 & -0.04003500 & 1.49194300 \\ \mathrm{C} & 1.49901400 & -0.01072700 & -0.67923100 \\ \mathrm{C} & 2.65100800 & 0.02929500 & 0.08038900 \\ \mathrm{C} & 1.25226800 & -0.25649800 & 3.57917800 \\ \mathrm{C} & 2.39065900 & -0.86326900 & 4.17231200 \\ \mathrm{C} & 3.66952000 & -0.73647400 & 3.53883800 \\ \mathrm{C} & 3.77705100 & -0.27282000 & 2.26151400 \\ \mathrm{C} & 0.01985800 & -0.21449100 & 4.35502300 \\ \mathrm{C} & -0.17216900 & -1.35573400 & 5.19063300 \\ \mathrm{C} & 0.95534100 & -2.10596100 & 5.64679900 \\ \mathrm{C} & 2.22096600 & -1.74455100 & 5.28116200 \\ \mathrm{C} & -0.94271400 & 0.88834800 & 4.52699900 \\ \mathrm{C} & -2.19001400 & 0.54591200 & 5.14253700 \\ \mathrm{C} & -2.50036600 & -0.80235900 & 5.49798900 \\ \mathrm{C} & -1.48156600 & -1.69568200 & 5.63642900 \\ \mathrm{C} & -0.55360300 & 2.30832600 & 4.59797900 \\ \mathrm{C} & -1.17752500 & 3.08307600 & 5.62177400 \\ & & & \mathrm{~S} 36\end{array}$




$\begin{array}{rrrr}\mathrm{C} & -2.48527000 & 2.74148800 & 6.07143200 \\ \mathrm{C} & -3.03046800 & 1.54143200 & 5.72841300 \\ \mathrm{C} & 0.50394400 & 3.01575800 & 3.88925500 \\ \mathrm{C} & 1.29849200 & 3.93369700 & 4.62559600 \\ \mathrm{C} & 0.81945700 & 4.43829700 & 5.87079900 \\ \mathrm{C} & -0.46027200 & 4.14736400 & 6.25014100 \\ \mathrm{C} & 0.59592900 & 3.10927400 & 2.44525800 \\ \mathrm{C} & 1.71091900 & 3.77991400 & 1.84931900 \\ \mathrm{C} & 2.71492900 & 4.35298000 & 2.69199700 \\ \mathrm{C} & 2.47801700 & 4.48527000 & 4.02774200 \\ \mathrm{C} & -0.51011100 & 2.80288000 & 1.61523200 \\ \mathrm{C} & -0.50407300 & 3.10906400 & 0.26857200 \\ \mathrm{C} & 0.63392800 & 3.68360100 & -0.32876600 \\ \mathrm{C} & 1.72664200 & 4.00168900 & 0.45256600 \\ \mathrm{H} & 1.55777000 & -0.00234200 & -1.76429100 \\ \mathrm{H} & 3.62675900 & 0.04997300 & -0.39983500 \\ \mathrm{H} & 4.74629400 & -0.20171200 & 1.77404500 \\ \mathrm{H} & 4.54807800 & -1.07517500 & 4.08294400 \\ \mathrm{H} & 3.09395000 & -2.22481200 & 5.71541000 \\ \mathrm{H} & 0.78286700 & -2.90918800 & 6.35869900 \\ \mathrm{H} & -1.63643300 & -2.65630200 & 6.12144200 \\ \mathrm{H} & -3.49944800 & -1.03196500 & 5.85887200 \\ \mathrm{H} & -4.01549600 & 1.25154300 & 6.08446300 \\ \mathrm{H} & -3.01399900 & 3.43620300 & 6.71938700 \\ \mathrm{H} & -0.92685400 & 4.65443800 & 7.09093900 \\ \mathrm{H} & 1.42148500 & 5.15900500 & 6.41804200 \\ \mathrm{H} & 3.15093700 & 5.06292400 & 4.65711000 \\ \mathrm{H} & 3.59783000 & 4.79411100 & 2.23588800 \\ \mathrm{H} & 2.59392900 & 4.48985500 & 0.01367700 \\ \mathrm{H} & -0.63987700 & 3.90139000 & -1.39339000 \\ \mathrm{H} & -1.38266900 & 2.89668000 & -0.33477800 \\ \mathrm{H} & -39669200 & 2.36932100 & 2.06622800 \\ \mathrm{H} & -0.17656500 & -0.63430700 \\ \mathrm{H} & -0.16591100 & 1.81919800\end{array}$

\section{( $M, M)$-PD8H-6R}

$\begin{array}{lrrr}\mathrm{C} & 1.18111000 & 0.21213000 & -0.39579300 \\ \mathrm{C} & 1.15531800 & 1.63968100 & -0.45653100 \\ \mathrm{C} & 0.00206500 & 2.34239700 & 0.00157500 \\ \mathrm{C} & -1.15229600 & 1.64169500 & 0.45943300 \\ \mathrm{C} & -0.00042100 & -0.48735700 & 0.00135900 \\ \mathrm{C} & -1.18086200 & 0.21422400 & 0.39869400 \\ \mathrm{C} & 2.26046000 & 2.34443200 & -0.99381300 \\ \mathrm{C} & 2.20479000 & 3.75981000 & -1.02463100 \\ \mathrm{C} & 1.11990700 & 4.45146600 & -0.52273200 \\ & & & \mathrm{~S} 37\end{array}$




\begin{tabular}{|c|c|c|c|}
\hline $\mathrm{C}$ & 0.00329500 & 3.75610600 & 0.00134100 \\
\hline $\mathrm{C}$ & -1.11157500 & 4.45511400 & 0.52514700 \\
\hline $\mathrm{C}$ & -2.19734000 & 3.76418600 & 1.02709000 \\
\hline $\mathrm{C}$ & -2.25606000 & 2.34903600 & 0.99604800 \\
\hline $\mathrm{C}$ & 1.12929000 & 5.93152500 & -0.52922900 \\
\hline $\mathrm{N}$ & 0.00335400 & 6.57899800 & -0.00162300 \\
\hline $\mathrm{O}$ & 2.06378900 & 6.59294900 & -0.97023600 \\
\hline $\mathrm{C}$ & -1.12516100 & 5.93689400 & 0.53535600 \\
\hline $\mathrm{O}$ & -2.06510800 & 6.58362400 & 0.98589400 \\
\hline $\mathrm{C}$ & 2.36210600 & -0.49821700 & -0.74157900 \\
\hline $\mathrm{C}$ & 2.27468300 & -1.91147500 & -0.83906900 \\
\hline $\mathrm{C}$ & 1.13787500 & -2.59757700 & -0.46523600 \\
\hline $\mathrm{C}$ & -0.00202900 & -1.89895000 & 0.00072600 \\
\hline $\mathrm{C}$ & -1.14401200 & -2.59385700 & 0.46617000 \\
\hline $\mathrm{C}$ & -2.27948600 & -1.90682200 & 0.84100200 \\
\hline $\mathrm{C}$ & -2.36365700 & -0.49332000 & 0.74422900 \\
\hline $\mathrm{C}$ & 1.11978900 & -4.07747100 & -0.54864500 \\
\hline $\mathrm{N}$ & -0.00544500 & -4.71998300 & -0.00370700 \\
\hline $\mathrm{O}$ & 2.03719100 & -4.72310100 & -1.04335600 \\
\hline $\mathrm{C}$ & -1.12406700 & -4.07238200 & 0.54056700 \\
\hline $\mathrm{O}$ & -2.03613000 & -4.73341500 & 1.02525600 \\
\hline $\mathrm{C}$ & 0.03463500 & 8.04650700 & -0.01792100 \\
\hline $\mathrm{H}$ & -0.91297100 & 8.40429900 & 0.37631600 \\
\hline $\mathrm{H}$ & 0.86187500 & 8.40838600 & 0.59774800 \\
\hline $\mathrm{H}$ & 0.18214200 & 8.40004000 & -1.04058400 \\
\hline $\mathrm{C}$ & -0.03907700 & -6.18696900 & 0.00803500 \\
\hline $\mathrm{H}$ & 0.88741200 & -6.54474400 & -0.43372900 \\
\hline $\mathrm{H}$ & -0.13754200 & -6.54656800 & 1.03475800 \\
\hline $\mathrm{H}$ & -0.89646800 & -6.54369300 & -0.56799500 \\
\hline $\mathrm{H}$ & 3.03481100 & 4.34475200 & -1.40065100 \\
\hline $\mathrm{H}$ & -3.02637400 & 4.35010400 & 1.40343000 \\
\hline $\mathrm{H}$ & 3.10436900 & -2.48681400 & -1.22398000 \\
\hline $\mathrm{H}$ & -3.11034900 & -2.48130500 & 1.22487200 \\
\hline $\mathrm{C}$ & -3.43149700 & 1.61614700 & 1.43329800 \\
\hline $\mathrm{C}$ & -3.56725500 & 0.24154400 & 1.10737500 \\
\hline $\mathrm{C}$ & -4.86713700 & -0.38394300 & 1.26953500 \\
\hline $\mathrm{C}$ & -5.76327900 & 0.21316200 & 2.20185100 \\
\hline $\mathrm{C}$ & -4.44591000 & 2.25087200 & 2.20800100 \\
\hline $\mathrm{C}$ & -5.51933500 & 1.53763200 & 2.66191600 \\
\hline $\mathrm{C}$ & -5.32699600 & -1.56699800 & 0.55851400 \\
\hline $\mathrm{C}$ & -6.34696300 & -2.34434900 & 1.18081400 \\
\hline $\mathrm{C}$ & -7.12040200 & -1.79000600 & 2.24467800 \\
\hline $\mathrm{C}$ & -6.89806400 & -0.51291200 & 2.67116500 \\
\hline $\mathrm{C}$ & -4.82244900 & -2.02299400 & -0.73015700 \\
\hline
\end{tabular}




\begin{tabular}{|c|c|c|c|}
\hline $\mathrm{C}$ & -4.94454100 & -3.41560600 & -1.01526900 \\
\hline $\mathrm{C}$ & -5.83864100 & -4.22678100 & -0.25381200 \\
\hline $\mathrm{C}$ & -6.59275600 & -3.68016900 & 0.74302200 \\
\hline $\mathrm{C}$ & -4.20284300 & -1.18588100 & -1.74265200 \\
\hline $\mathrm{C}$ & -3.35009900 & -1.81273300 & -2.69184800 \\
\hline $\mathrm{C}$ & -3.32582500 & -3.23359600 & -2.80791000 \\
\hline $\mathrm{C}$ & -4.17460900 & -4.00042400 & -2.06218300 \\
\hline $\mathrm{C}$ & -4.47515300 & 0.23392100 & -1.92929900 \\
\hline $\mathrm{C}$ & -3.64889500 & 0.99501600 & -2.81631700 \\
\hline $\mathrm{C}$ & -2.63840900 & 0.33691800 & -3.58566300 \\
\hline $\mathrm{C}$ & -2.54213200 & -1.02127600 & -3.57016700 \\
\hline $\mathrm{C}$ & -5.60540700 & 0.88947100 & -1.37536500 \\
\hline $\mathrm{C}$ & -5.84730100 & 2.23162600 & -1.59655800 \\
\hline $\mathrm{C}$ & -4.96525600 & 2.99802800 & -2.38498400 \\
\hline $\mathrm{C}$ & -3.89264200 & 2.37980500 & -2.99308200 \\
\hline $\mathrm{H}$ & -4.32250900 & 3.28135300 & 2.51913700 \\
\hline $\mathrm{H}$ & -6.22955900 & 1.98913900 & 3.34935300 \\
\hline $\mathrm{H}$ & -7.54416100 & -0.04844200 & 3.41120100 \\
\hline $\mathrm{H}$ & -7.92247800 & -2.38846200 & 2.66839900 \\
\hline $\mathrm{H}$ & -7.34696500 & -4.26689300 & 1.26046200 \\
\hline $\mathrm{H}$ & -5.94354000 & -5.27457500 & -0.52215100 \\
\hline $\mathrm{H}$ & -4.24745600 & -5.07268300 & -2.22301800 \\
\hline $\mathrm{H}$ & -2.67819700 & -3.68436800 & -3.55542700 \\
\hline $\mathrm{H}$ & -1.84558400 & -1.53525200 & -4.22773000 \\
\hline $\mathrm{H}$ & -2.00593900 & 0.93329900 & -4.23829300 \\
\hline $\mathrm{H}$ & -3.23288600 & 2.94113700 & -3.65035300 \\
\hline $\mathrm{H}$ & -5.14993700 & 4.05741700 & -2.53963300 \\
\hline $\mathrm{H}$ & -6.73033100 & 2.69501000 & -1.16521900 \\
\hline $\mathrm{H}$ & -6.31576500 & 0.32018800 & -0.79008400 \\
\hline $\mathrm{C}$ & 3.43375100 & 1.60887100 & -1.43206800 \\
\hline $\mathrm{C}$ & 3.56698600 & 0.23404000 & -1.10608400 \\
\hline $\mathrm{C}$ & 4.86552200 & -0.39386700 & -1.26960600 \\
\hline $\mathrm{C}$ & 5.76165200 & 0.20120000 & -2.20324700 \\
\hline $\mathrm{C}$ & 4.44844000 & 2.24148600 & -2.20815300 \\
\hline $\mathrm{C}$ & 5.51977900 & 1.52601300 & -2.66342000 \\
\hline $\mathrm{C}$ & 5.32422100 & -1.57719400 & -0.55837000 \\
\hline $\mathrm{C}$ & 6.34238400 & -2.35654500 & -1.18114600 \\
\hline $\mathrm{C}$ & 7.11534000 & -1.80429100 & -2.24643700 \\
\hline $\mathrm{C}$ & 6.89455100 & -0.52714700 & -2.67357400 \\
\hline $\mathrm{C}$ & 4.82040900 & -2.03127900 & 0.73122500 \\
\hline $\mathrm{C}$ & 4.94122800 & -3.42372000 & 1.01768400 \\
\hline $\mathrm{C}$ & 5.83354400 & -4.23676800 & 0.25615000 \\
\hline $\mathrm{C}$ & 6.58704700 & -3.69220100 & -0.74225600 \\
\hline $\mathrm{C}$ & 4.20284600 & -1.19242800 & 1.74354200 \\
\hline
\end{tabular}




$\begin{array}{lrrr}\mathrm{C} & 3.35016300 & -1.81731400 & 2.69408400 \\ \mathrm{C} & 3.32442100 & -3.23801400 & 2.81164500 \\ \mathrm{C} & 4.17166400 & -4.00655700 & 2.06595400 \\ \mathrm{C} & 4.47720900 & 0.22716900 & 1.92877200 \\ \mathrm{C} & 3.65263600 & 0.99014700 & 2.81574500 \\ \mathrm{C} & 2.64185500 & 0.33406500 & 3.58643300 \\ \mathrm{C} & 2.54384900 & -1.02401700 & 3.57227500 \\ \mathrm{C} & 5.60794500 & 0.88069900 & 1.37344400 \\ \mathrm{C} & 5.85180200 & 2.22272200 & 1.59326300 \\ \mathrm{C} & 4.97136400 & 2.99100600 & 2.38164800 \\ \mathrm{C} & 3.89836800 & 2.37476400 & 2.99109300 \\ \mathrm{H} & 4.32660900 & 3.27218700 & -2.51918400 \\ \mathrm{H} & 6.23000900 & 1.97591900 & -3.35189700 \\ \mathrm{H} & 7.54051000 & -0.06424700 & -3.41471400 \\ \mathrm{H} & 7.91594300 & -2.40434000 & -2.67069100 \\ \mathrm{H} & 7.33993500 & -4.28039300 & -1.25995500 \\ \mathrm{H} & 5.93758700 & -5.28435900 & 0.52562500 \\ \mathrm{H} & 4.24342000 & -5.07872400 & 2.22784100 \\ \mathrm{H} & 2.67687100 & -3.68733200 & 3.56010300 \\ \mathrm{H} & 1.84717000 & -1.53651400 & 4.23085100 \\ \mathrm{H} & 2.01064300 & 0.93186400 & 4.23898300 \\ \mathrm{H} & 3.23985100 & 2.93756800 & 3.64834700 \\ \mathrm{H} & 5.15757300 & 4.05028600 & 2.53520800 \\ \mathrm{H} & 6.73512600 & 2.68453900 & 1.16085100 \\ \mathrm{H} & 6.31709500 & 0.30994900 & 0.78812000\end{array}$

\section{( $P, M)$-PD8H-6M}

$\begin{array}{lrcc}\mathrm{C} & -1.27845100 & 0.35856800 & -1.02199800 \\ \mathrm{C} & -1.27828300 & 1.78479400 & -0.92424300 \\ \mathrm{C} & -0.03877000 & 2.48947300 & -0.85858000 \\ \mathrm{C} & 1.20707400 & 1.79551100 & -0.92205800 \\ \mathrm{C} & -0.02549900 & -0.33045800 & -1.10997300 \\ \mathrm{C} & 1.22159500 & 0.36930000 & -1.02066000 \\ \mathrm{C} & -2.50136200 & 2.49698800 & -0.99724400 \\ \mathrm{C} & -2.46892100 & 3.90893200 & -0.89034600 \\ \mathrm{C} & -1.27891600 & 4.59700600 & -0.77370200 \\ \mathrm{C} & -0.04536300 & 3.90278800 & -0.79168800 \\ \mathrm{C} & 1.18129700 & 4.60956700 & -0.76677400 \\ \mathrm{C} & 2.37725900 & 3.93109500 & -0.88124000 \\ \mathrm{C} & 2.42271000 & 2.51989200 & -0.99313600 \\ \mathrm{C} & -1.29695000 & 6.07228700 & -0.65173000 \\ \mathrm{~N} & -0.05731500 & 6.72236400 & -0.58720000 \\ \mathrm{O} & -2.33343900 & 6.72707000 & -0.60686400 \\ \mathrm{C} & 1.19367200 & 6.08638300 & -0.64190100 \\ \mathrm{O} & 2.23437800 & 6.73346000 & -0.58903800\end{array}$




$\begin{array}{lrrr}\mathrm{C} & -2.50957900 & -0.35594300 & -1.07858000 \\ \mathrm{C} & -2.44689500 & -1.74238000 & -1.37389600 \\ \mathrm{C} & -1.24785800 & -2.41211400 & -1.49580900 \\ \mathrm{C} & -0.01991300 & -1.72542100 & -1.33612300 \\ \mathrm{C} & 1.21440200 & -2.40086600 & -1.48889300 \\ \mathrm{C} & 2.40807100 & -1.72224300 & -1.36656400 \\ \mathrm{C} & 2.46013200 & -0.33367900 & -1.07850100 \\ \mathrm{C} & -1.25994600 & -3.85601700 & -1.83397300 \\ \mathrm{~N} & -0.00865700 & -4.48262100 & -1.95404500 \\ \mathrm{O} & -2.29820300 & -4.48600500 & -2.00365100 \\ \mathrm{C} & 1.23172500 & -3.84382400 & -1.82372200 \\ \mathrm{O} & 2.26579800 & -4.48252900 & -1.98807700 \\ \mathrm{C} & -0.09796600 & 8.18436500 & -0.46294500 \\ \mathrm{H} & 0.92722800 & 8.54444300 & -0.43558300 \\ \mathrm{H} & -0.63011500 & 8.61347500 & -1.31499800 \\ \mathrm{H} & -0.62451700 & 8.46394000 & 0.45257000 \\ \mathrm{C} & 0.03137100 & -5.91270300 & -2.28035300 \\ \mathrm{C} & 3.73531900 & -4.24232100 & 1.63775200 \\ \mathrm{H} & -0.99170900 & -6.27951600 & -2.29460000 \\ \mathrm{H} & 0.49937200 & -6.06120000 & -3.25689000 \\ \mathrm{H} & 0.61975500 & -6.44362200 & -1.52915000 \\ \mathrm{C} & 3.26255700 & 0.63289000 & 3.00172400 \\ \mathrm{H} & -3.38252700 & 4.48973400 & -0.87671300 \\ \mathrm{C} & 3.28602100 & 4.51913300 & -0.86331100 \\ \mathrm{C} & & & \end{array}$




\begin{tabular}{|c|c|c|c|}
\hline $\mathrm{C}$ & 2.16037200 & -0.08308000 & 3.56508100 \\
\hline $\mathrm{C}$ & 2.03718900 & -1.42273300 & 3.35712600 \\
\hline $\mathrm{C}$ & 5.38457900 & 0.63358000 & 1.81885400 \\
\hline $\mathrm{C}$ & 5.62577300 & 1.92703700 & 2.24033100 \\
\hline $\mathrm{C}$ & 4.66494600 & 2.62392500 & 3.00040500 \\
\hline $\mathrm{C}$ & 3.51111200 & 1.97433400 & 3.38583400 \\
\hline $\mathrm{H}$ & 4.79559800 & 3.57890300 & -1.77354900 \\
\hline $\mathrm{H}$ & 6.81677800 & 2.34607600 & -2.39052200 \\
\hline $\mathrm{H}$ & 8.05941300 & 0.27365500 & -2.56795000 \\
\hline $\mathrm{H}$ & 8.23404800 & -2.17423700 & -2.16930800 \\
\hline $\mathrm{H}$ & 7.36332200 & -4.21254200 & -1.18914700 \\
\hline $\mathrm{H}$ & 5.66137400 & -5.38419100 & 0.18850800 \\
\hline $\mathrm{H}$ & 3.74972300 & -5.32879900 & 1.65293600 \\
\hline $\mathrm{H}$ & 2.07463800 & -4.05590800 & 2.96926700 \\
\hline $\mathrm{H}$ & 1.27157300 & -1.99626100 & 3.87201000 \\
\hline $\mathrm{H}$ & 1.47811000 & 0.44464300 & 4.22656200 \\
\hline $\mathrm{H}$ & 2.78583500 & 2.47185400 & 4.02527400 \\
\hline $\mathrm{H}$ & 4.85054300 & 3.64814300 & 3.31154300 \\
\hline $\mathrm{H}$ & 6.56958000 & 2.40378900 & 1.99065700 \\
\hline $\mathrm{H}$ & 6.15556900 & 0.11353800 & 1.26653600 \\
\hline $\mathrm{C}$ & -3.74643700 & 1.77189600 & -1.17064100 \\
\hline $\mathrm{C}$ & -3.77812900 & 0.36234200 & -1.01271800 \\
\hline $\mathrm{C}$ & -5.07489100 & -0.29554300 & -0.96462400 \\
\hline $\mathrm{C}$ & -6.16820600 & 0.37586700 & -1.58276600 \\
\hline $\mathrm{C}$ & -4.92895500 & 2.45890500 & -1.57619100 \\
\hline $\mathrm{C}$ & -6.06561600 & 1.76413800 & -1.87538100 \\
\hline $\mathrm{C}$ & -5.35089800 & -1.58439200 & -0.35136700 \\
\hline $\mathrm{C}$ & -6.45781800 & -2.32661200 & -0.85674300 \\
\hline $\mathrm{C}$ & -7.45265400 & -1.67520600 & -1.64553000 \\
\hline $\mathrm{C}$ & -7.35935000 & -0.33915700 & -1.90797400 \\
\hline $\mathrm{C}$ & -4.58500500 & -2.17319600 & 0.73641300 \\
\hline $\mathrm{C}$ & -4.61062700 & -3.59494100 & 0.85111400 \\
\hline $\mathrm{C}$ & -5.61425200 & -4.34922100 & 0.17238600 \\
\hline $\mathrm{C}$ & -6.56990500 & -3.72026500 & -0.57131600 \\
\hline $\mathrm{C}$ & -3.80509500 & -1.43435700 & 1.71183900 \\
\hline $\mathrm{C}$ & -2.77319900 & -2.12902500 & 2.39876500 \\
\hline $\mathrm{C}$ & -2.68616900 & -3.54982900 & 2.32000100 \\
\hline $\mathrm{C}$ & -3.63621200 & -4.26327600 & 1.64719200 \\
\hline $\mathrm{C}$ & -4.07598000 & -0.06316300 & 2.12852200 \\
\hline $\mathrm{C}$ & -3.11333600 & 0.62721400 & 2.93098400 \\
\hline $\mathrm{C}$ & -1.96260300 & -0.07055600 & 3.41470200 \\
\hline $\mathrm{C}$ & -1.84056100 & -1.41128600 & 3.21301000 \\
\hline $\mathrm{C}$ & -5.31101900 & 0.59470500 & 1.89286500 \\
\hline $\mathrm{C}$ & -5.53822100 & 1.88872200 & 2.32044500 \\
\hline
\end{tabular}




$\begin{array}{lrrr}\text { C } & -4.53656100 & 2.60159200 & 3.01000300 \\ \text { C } & -3.35118700 & 1.96880300 & 3.32079300 \\ \text { H } & -4.89818400 & 3.52660300 & -1.75542500 \\ \text { H } & -6.92379200 & 2.27087300 & -2.30854900 \\ \text { H } & -8.16033100 & 0.18956200 & -2.41759300 \\ \text { H } & -8.31012500 & -2.25244400 & -1.98092600 \\ \text { H } & -7.39687900 & -4.27541100 & -1.00584000 \\ \text { H } & -5.63314300 & -5.42713900 & 0.30854600 \\ \text { H } & -3.64353800 & -5.34961200 & 1.67431100 \\ \text { H } & -1.89569000 & -4.05481100 & 2.86906500 \\ \text { H } & -1.02579100 & -1.96858900 & 3.66579700 \\ \text { H } & -1.23463300 & 0.47264400 & 4.01161500 \\ \text { H } & -2.58990600 & 2.48048400 & 3.90481900 \\ \text { H } & -4.71294600 & 3.62600700 & 3.32585600 \\ \text { H } & -6.50130900 & 2.35416500 & 2.13004300 \\ \text { H } & -6.10956100 & 0.06202400 & 1.39348800\end{array}$

\section{TS of PD8H}

$\begin{array}{lrrr}\mathrm{C} & -1.19410800 & 1.60655500 & 0.53332700 \\ \mathrm{C} & -1.22885000 & 0.18133500 & 0.42663400 \\ \mathrm{C} & -0.05337500 & -0.51040100 & -0.00131000 \\ \mathrm{C} & 1.12516800 & 0.19904800 & -0.37292200 \\ \mathrm{C} & -0.05464400 & 2.31786500 & 0.05799700 \\ \mathrm{C} & 1.08280900 & 1.62290400 & -0.43891800 \\ \mathrm{C} & -2.41262700 & -0.53357800 & 0.75031600 \\ \mathrm{C} & -2.33927400 & -1.95203700 & 0.76209700 \\ \mathrm{C} & -1.21272000 & -2.62906600 & 0.34178700 \\ \mathrm{C} & -0.05883000 & -1.92060100 & -0.07307800 \\ \mathrm{C} & 1.09734500 & -2.59966600 & -0.52557800 \\ \mathrm{C} & 2.25559200 & -1.90674800 & -0.81597700 \\ \mathrm{C} & 2.31829200 & -0.49463500 & -0.71450700 \\ \mathrm{C} & -1.21977900 & -4.11223600 & 0.33300300 \\ \mathrm{~N} & -0.06914300 & -4.73673900 & -0.18559700 \\ \mathrm{O} & -2.16894700 & -4.77310800 & 0.73342900 \\ \mathrm{C} & 1.09454800 & -4.07734000 & -0.61098700 \\ \mathrm{O} & 2.05532400 & -4.72788500 & -1.00461200 \\ \mathrm{C} & -2.28590300 & 2.30369100 & 1.10341200 \\ \mathrm{C} & -2.22599300 & 3.71936800 & 1.15516100 \\ \mathrm{C} & -1.15885900 & 4.41861700 & 0.62481700 \\ \mathrm{C} & -0.05991100 & 3.73109400 & 0.05427900 \\ \mathrm{C} & 1.02284600 & 4.43248900 & -0.53335800 \\ \mathrm{C} & 2.07400600 & 3.74256800 & -1.11043000 \\ \mathrm{C} & 2.13708300 & 2.32940900 & -1.06398500 \\ \mathrm{C} & -1.17292200 & 5.90007300 & 0.63900100 \\ \mathrm{~N} & -0.07547300 & 6.55041700 & 0.05266000\end{array}$




\begin{tabular}{|c|c|c|c|}
\hline $\mathrm{O}$ & -2.08303700 & 6.55599000 & 1.12980200 \\
\hline $\mathrm{C}$ & 1.02811000 & 5.91539900 & -0.54761400 \\
\hline $\mathrm{O}$ & 1.93540100 & 6.56503100 & -1.05108000 \\
\hline $\mathrm{C}$ & -0.04227900 & -6.20114700 & -0.25482400 \\
\hline $\mathrm{H}$ & -1.02271000 & -6.56465000 & 0.04411600 \\
\hline $\mathrm{H}$ & 0.19696100 & -6.51530600 & -1.27306600 \\
\hline $\mathrm{H}$ & 0.72825700 & -6.59591300 & 0.41329400 \\
\hline $\mathrm{C}$ & -0.11040000 & 8.01683900 & 0.07280900 \\
\hline $\mathrm{H}$ & -0.17273300 & 8.37060900 & 1.10467300 \\
\hline $\mathrm{H}$ & 0.79943800 & 8.37593600 & -0.40239700 \\
\hline $\mathrm{H}$ & -0.99186200 & 8.37459300 & -0.46516600 \\
\hline $\mathrm{H}$ & -3.17009300 & -2.54470900 & 1.11698900 \\
\hline $\mathrm{H}$ & 3.11801200 & -2.46946500 & -1.15274800 \\
\hline $\mathrm{H}$ & -3.04459200 & 4.30251900 & 1.55952600 \\
\hline $\mathrm{H}$ & 2.87525100 & 4.32272400 & -1.55472700 \\
\hline $\mathrm{C}$ & 3.24123100 & 1.57600100 & -1.62929600 \\
\hline $\mathrm{C}$ & 3.47259000 & 0.24763500 & -1.18684300 \\
\hline $\mathrm{C}$ & 4.73546500 & -0.38357400 & -1.54872000 \\
\hline $\mathrm{C}$ & 5.20184900 & -0.02268300 & -2.84635900 \\
\hline $\mathrm{C}$ & 3.98877700 & 2.07913200 & -2.73306900 \\
\hline $\mathrm{C}$ & 4.80831100 & 1.22405100 & -3.41777400 \\
\hline $\mathrm{C}$ & 5.64991400 & -1.18206000 & -0.71863600 \\
\hline $\mathrm{C}$ & 6.68349000 & -1.88243000 & -1.42023400 \\
\hline $\mathrm{C}$ & 6.76250100 & -1.86050400 & -2.84670300 \\
\hline $\mathrm{C}$ & 6.12612200 & -0.86681200 & -3.52702400 \\
\hline $\mathrm{C}$ & 5.89868000 & -0.90742000 & 0.70697300 \\
\hline $\mathrm{C}$ & 7.26184600 & -0.94454700 & 1.12621500 \\
\hline $\mathrm{C}$ & 8.18749400 & -1.78976800 & 0.44980000 \\
\hline $\mathrm{C}$ & 7.85255200 & -2.34961100 & -0.74648300 \\
\hline $\mathrm{C}$ & 4.97957900 & -0.41929700 & 1.72411600 \\
\hline $\mathrm{C}$ & 5.44373000 & 0.58865700 & 2.60944900 \\
\hline $\mathrm{C}$ & 6.84666200 & 0.79920800 & 2.76109900 \\
\hline $\mathrm{C}$ & 7.71330800 & -0.07239000 & 2.16471400 \\
\hline $\mathrm{C}$ & 3.75909500 & -1.09814500 & 2.11354400 \\
\hline $\mathrm{C}$ & 2.88889800 & -0.49088600 & 3.07434300 \\
\hline $\mathrm{C}$ & 3.24789400 & 0.77234000 & 3.64260300 \\
\hline $\mathrm{C}$ & 4.51183700 & 1.25388800 & 3.47079300 \\
\hline $\mathrm{C}$ & 3.53887700 & -2.45977900 & 1.78945900 \\
\hline $\mathrm{C}$ & 2.50024800 & -3.17258800 & 2.35732000 \\
\hline $\mathrm{C}$ & 1.58834100 & -2.54070600 & 3.22483400 \\
\hline $\mathrm{C}$ & 1.77698900 & -1.21524400 & 3.56444900 \\
\hline $\mathrm{H}$ & 3.78294600 & 3.07227000 & -3.11722900 \\
\hline $\mathrm{H}$ & 5.24448500 & 1.51386700 & -4.37012400 \\
\hline $\mathrm{H}$ & 6.34092100 & -0.67255500 & -4.57466600 \\
\hline
\end{tabular}




\begin{tabular}{|c|c|c|c|}
\hline $\mathrm{H}$ & 7.49194000 & -2.49731000 & -3.33963600 \\
\hline $\mathrm{H}$ & 8.55704500 & -2.97324700 & -1.28992400 \\
\hline $\mathrm{H}$ & 9.17199000 & -1.94128500 & 0.88525400 \\
\hline $\mathrm{H}$ & 8.77468600 & -0.05865200 & 2.39861600 \\
\hline $\mathrm{H}$ & 7.19663700 & 1.55439000 & 3.45984300 \\
\hline $\mathrm{H}$ & 4.85590700 & 2.12208800 & 4.02771200 \\
\hline $\mathrm{H}$ & 2.55048000 & 1.26580800 & 4.31487200 \\
\hline $\mathrm{H}$ & 1.11042000 & -0.72868700 & 4.27274100 \\
\hline $\mathrm{H}$ & 0.75999200 & -3.10158800 & 3.64882600 \\
\hline $\mathrm{H}$ & 2.38837700 & -4.22782700 & 2.12443500 \\
\hline $\mathrm{H}$ & 4.23672600 & -2.96470400 & 1.12962400 \\
\hline $\mathrm{C}$ & -3.45195300 & 1.55804700 & 1.54278100 \\
\hline $\mathrm{C}$ & -3.60212700 & 0.19599400 & 1.17007500 \\
\hline $\mathrm{C}$ & -4.90365200 & -0.42479900 & 1.34003900 \\
\hline $\mathrm{C}$ & -5.77111400 & 0.14003000 & 2.31837600 \\
\hline $\mathrm{C}$ & -4.44349700 & 2.16913900 & 2.36396700 \\
\hline $\mathrm{C}$ & -5.50800600 & 1.44497200 & 2.82061600 \\
\hline $\mathrm{C}$ & -5.39240000 & -1.57330700 & 0.59340000 \\
\hline $\mathrm{C}$ & -6.39813300 & -2.37150600 & 1.21173900 \\
\hline $\mathrm{C}$ & -7.13748000 & -1.85721600 & 2.31841600 \\
\hline $\mathrm{C}$ & -6.89609200 & -0.59959500 & 2.78914600 \\
\hline $\mathrm{C}$ & -4.92753800 & -1.97626100 & -0.72715500 \\
\hline $\mathrm{C}$ & -5.06287800 & -3.35537000 & -1.06475800 \\
\hline $\mathrm{C}$ & -5.93667700 & -4.19425900 & -0.31123900 \\
\hline $\mathrm{C}$ & -6.66087100 & -3.68724000 & 0.72706800 \\
\hline $\mathrm{C}$ & -4.33088900 & -1.09966900 & -1.71942200 \\
\hline $\mathrm{C}$ & -3.50827800 & -1.68961600 & -2.71707800 \\
\hline $\mathrm{C}$ & -3.49470000 & -3.10402000 & -2.89208800 \\
\hline $\mathrm{C}$ & -4.32426200 & -3.89846400 & -2.15476000 \\
\hline $\mathrm{C}$ & -4.59660400 & 0.32854200 & -1.83579700 \\
\hline $\mathrm{C}$ & -3.78823500 & 1.12266200 & -2.70959800 \\
\hline $\mathrm{C}$ & -2.80507900 & 0.49211100 & -3.53442200 \\
\hline $\mathrm{C}$ & -2.71894500 & -0.86552500 & -3.58133600 \\
\hline $\mathrm{C}$ & -5.70417400 & 0.96693600 & -1.22087700 \\
\hline $\mathrm{C}$ & -5.93740300 & 2.31991500 & -1.37082300 \\
\hline $\mathrm{C}$ & -5.07101000 & 3.11432300 & -2.14777200 \\
\hline $\mathrm{C}$ & -4.02358900 & 2.51556500 & -2.81512000 \\
\hline $\mathrm{H}$ & -4.30294400 & 3.18764700 & 2.70675400 \\
\hline $\mathrm{H}$ & -6.19766400 & 1.87358400 & 3.54328700 \\
\hline $\mathrm{H}$ & -7.51840100 & -0.16244900 & 3.56569800 \\
\hline $\mathrm{H}$ & -7.93067200 & -2.46899500 & 2.74039900 \\
\hline $\mathrm{H}$ & -7.40098900 & -4.29345800 & 1.24279000 \\
\hline $\mathrm{H}$ & -6.04929200 & -5.23106900 & -0.61648800 \\
\hline $\mathrm{H}$ & -4.40238800 & -4.96383600 & -2.35410800 \\
\hline
\end{tabular}




$\begin{array}{lrrr}\mathrm{H} & -2.86953500 & -3.52572700 & -3.67510100 \\ \mathrm{H} & -2.04322000 & -1.35483800 & -4.27849100 \\ \mathrm{H} & -2.18552100 & 1.11263600 & -4.17706200 \\ \mathrm{H} & -3.37718600 & 3.10138900 & -3.46444600 \\ \mathrm{H} & -5.24834300 & 4.18165600 & -2.24671300 \\ \mathrm{H} & -6.80244900 & 2.77035700 & -0.89187800 \\ \mathrm{H} & -6.40362400 & 0.37629100 & -0.64382900\end{array}$




\section{NMR Spectra}

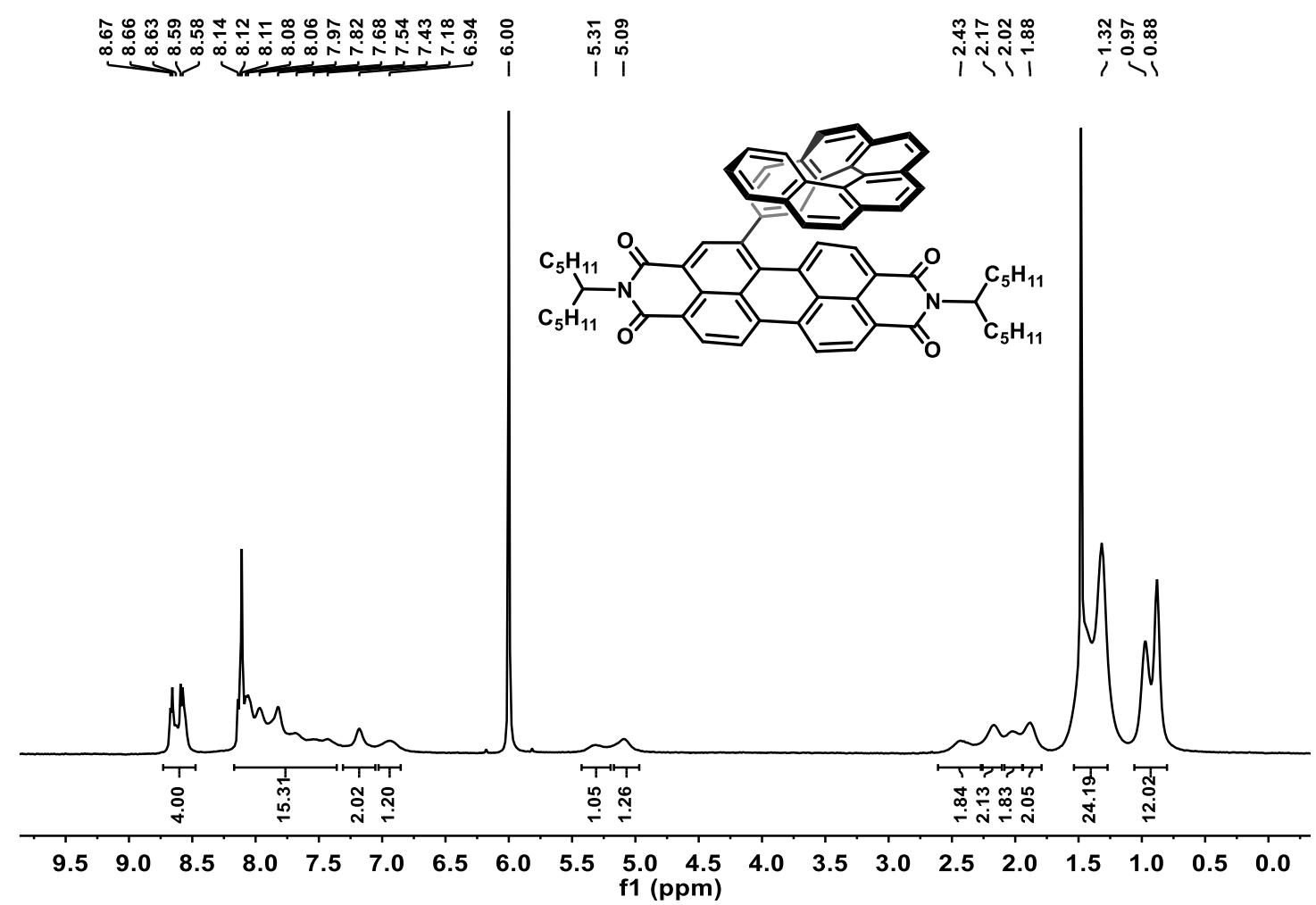

Figure S26. ${ }^{1} \mathrm{H}$ NMR spectrum of compound rac-5 $\left(500 \mathrm{MHz}, \mathrm{C}_{2} \mathrm{D}_{2} \mathrm{Cl}_{4}, 373.2 \mathrm{~K}\right)$

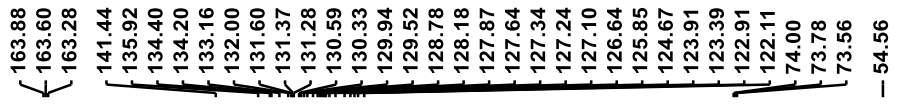

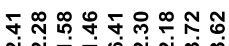

กู่
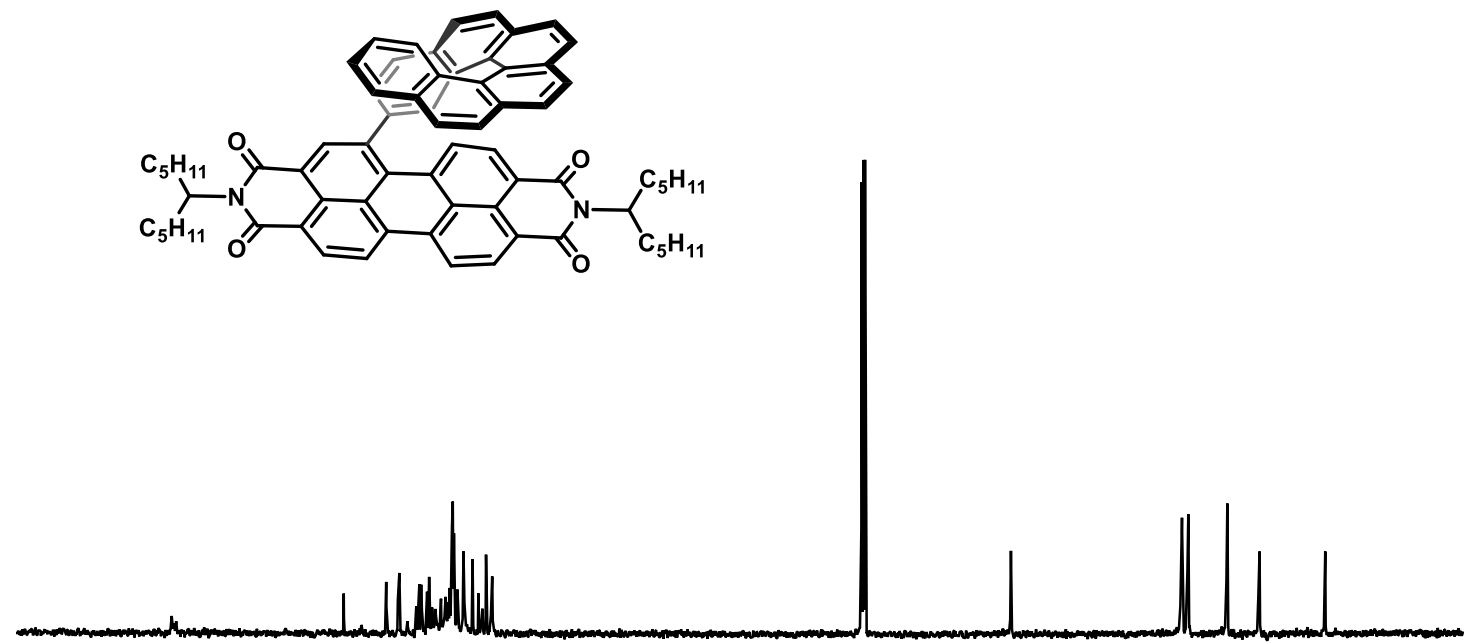

$\begin{array}{lllllllllllllllllll}180 & 170 & 160 & 150 & 140 & 130 & 120 & 110 & 100 \underset{f 1}{(\mathrm{ppm})} & 80 & 70 & 60 & 50 & 40 & 30 & 20 & 10 & 0\end{array}$

Figure S27. ${ }^{13} \mathrm{C}$ NMR spectrum of compound rac -5 (125 MHz, $\left.\mathrm{C}_{2} \mathrm{D}_{2} \mathrm{Cl}_{4}, 373.2 \mathrm{~K}\right)$ 


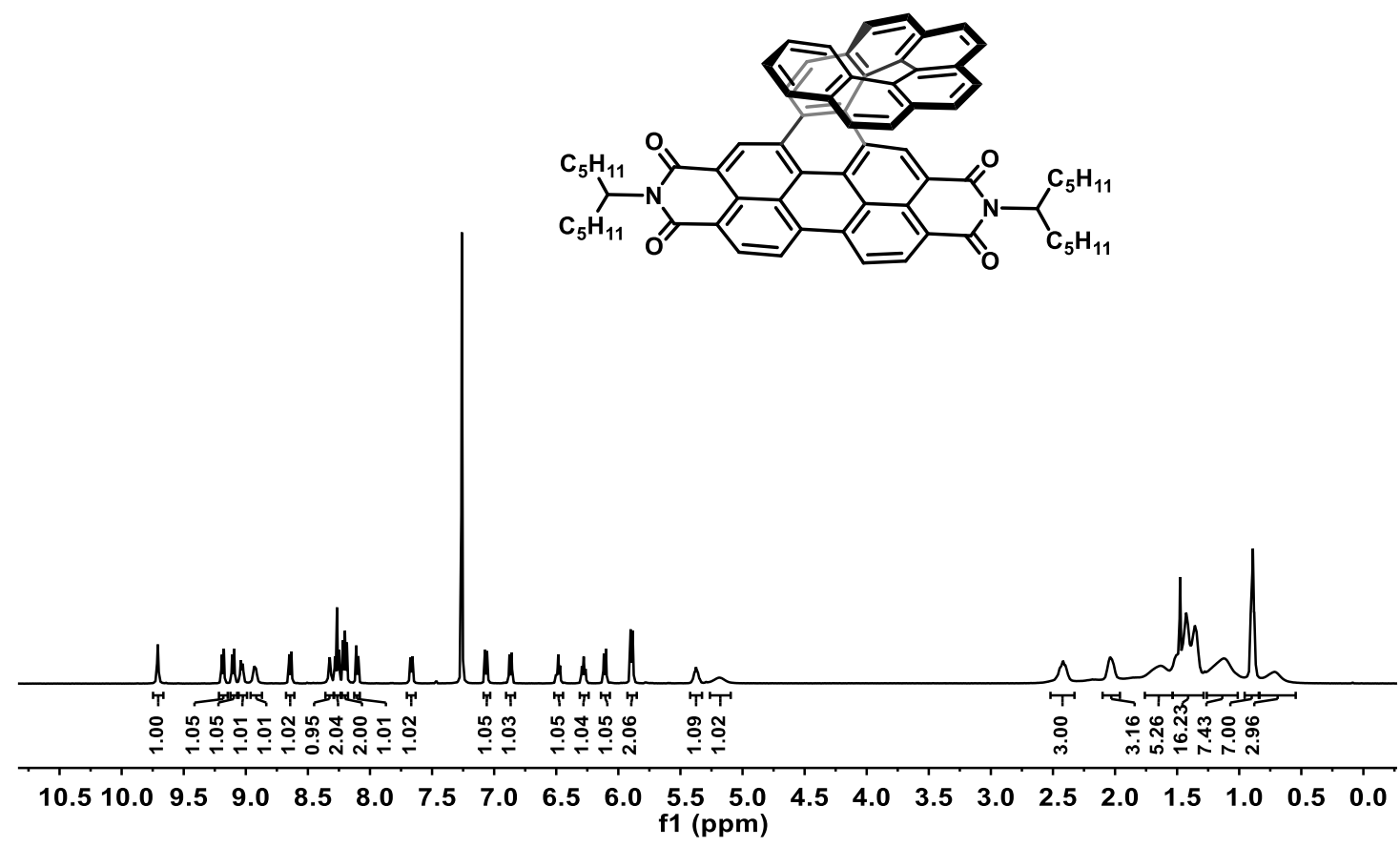

Figure S28. ${ }^{1} \mathrm{H}$ NMR spectrum of compound $\mathbf{P 8 H}\left(500 \mathrm{MHz}, \mathrm{CDCl}_{3}, 328.2 \mathrm{~K}\right)$

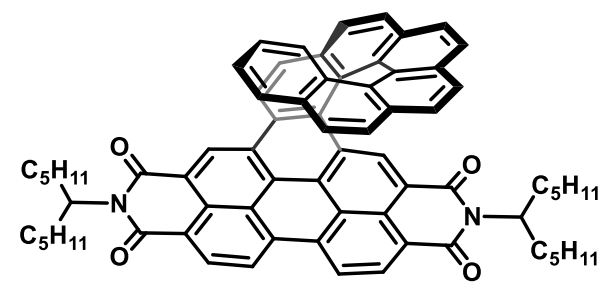

$\begin{array}{lllllllllll}180 & 170 & 160 & 150 & 140 & 130 & 120 & 110 & 100 & 90 & 90 \\ \mathrm{f} 1(\mathrm{ppm})\end{array}$

Figure S29. ${ }^{13} \mathrm{C}$ NMR spectrum of compound P8H $\left(125 \mathrm{MHz}, \mathrm{CDCl}_{3}, 328.2 \mathrm{~K}\right)$ 


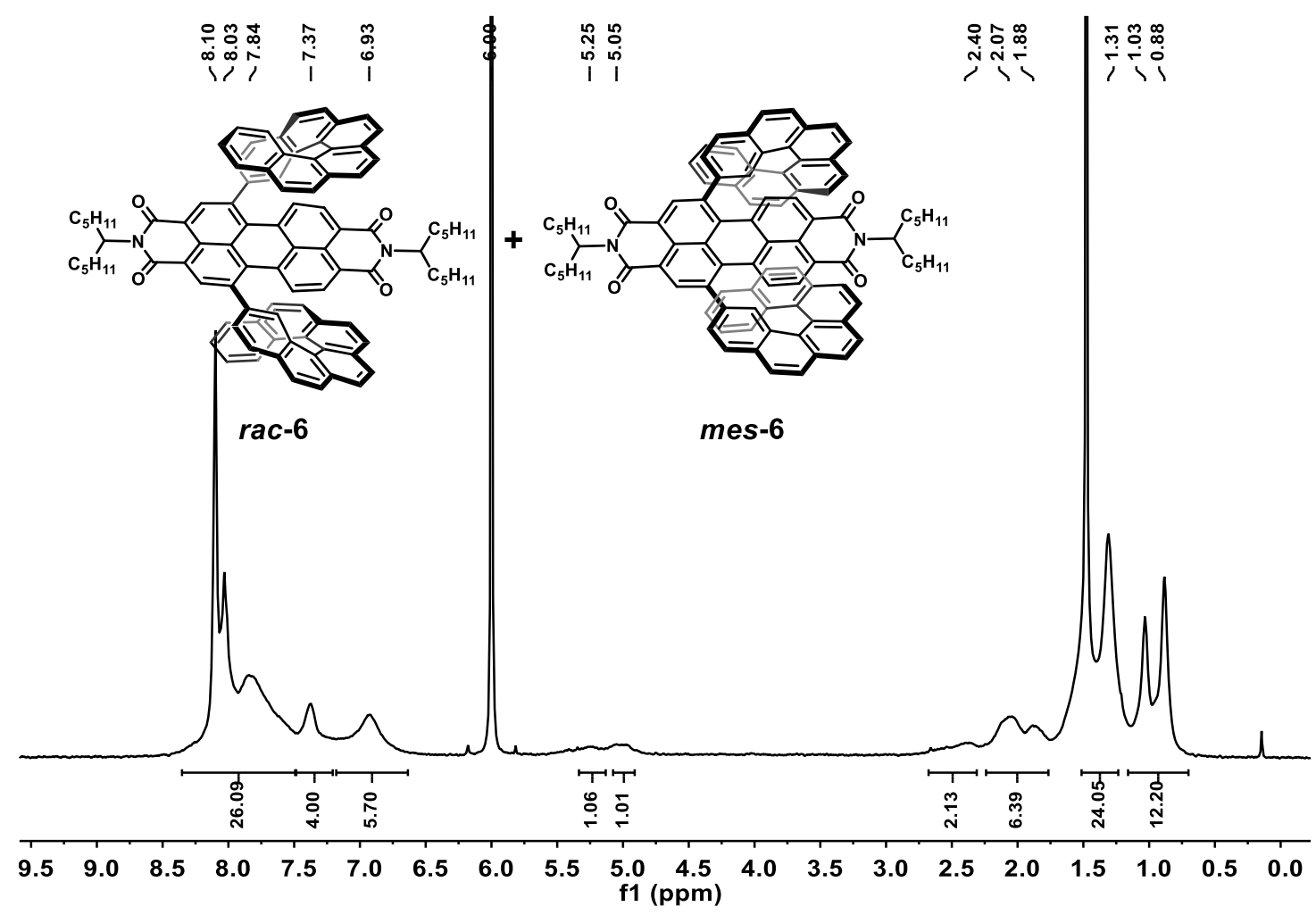

Figure S30. ${ }^{1} \mathrm{H}$ NMR spectrum of compound $\boldsymbol{r a c}-6$ and $\boldsymbol{m e s}-\mathbf{6}\left(500 \mathrm{MHz}, \mathrm{C}_{2} \mathrm{D}_{2} \mathrm{Cl}_{4}, 373.2 \mathrm{~K}\right)$

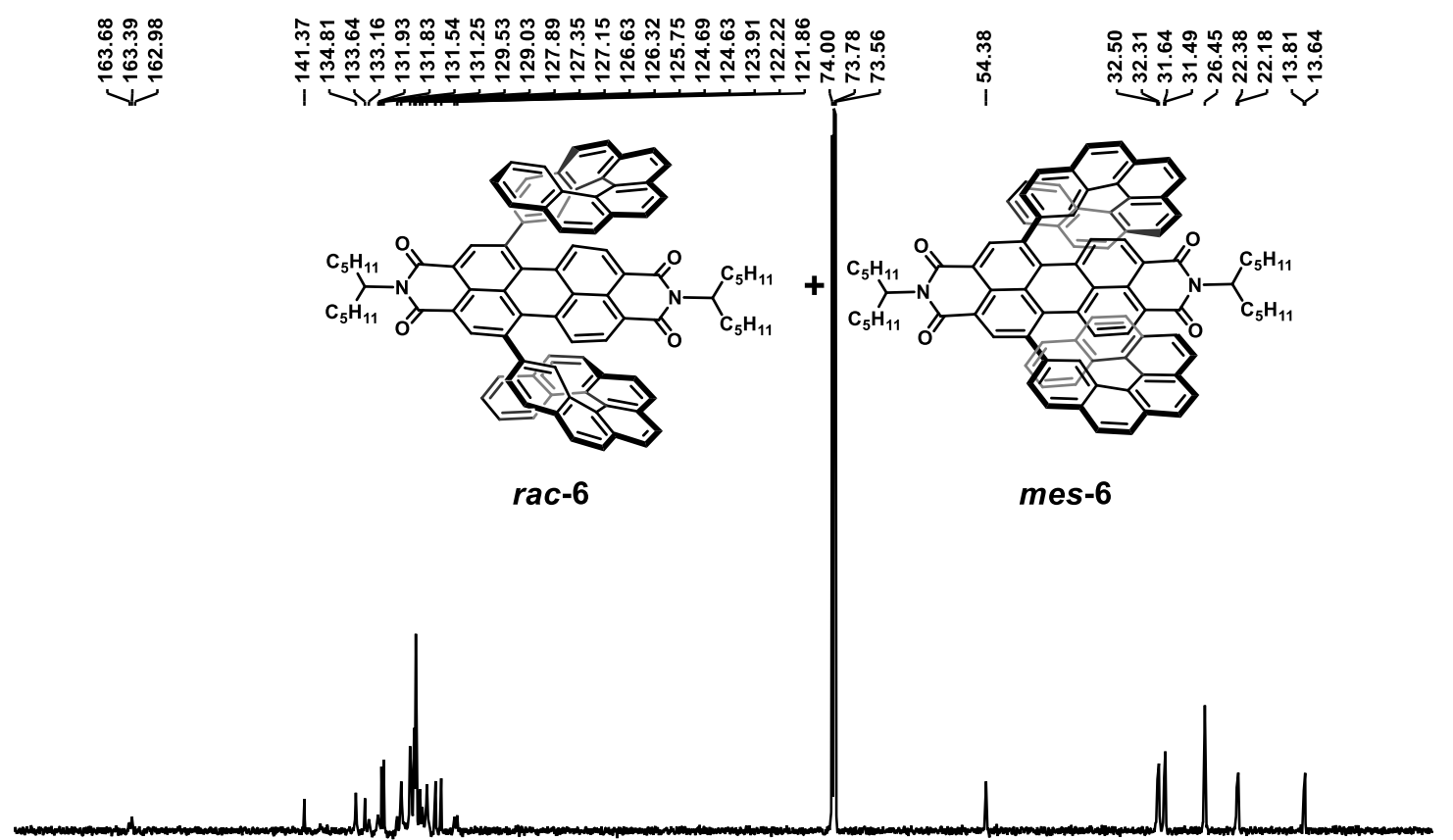

$\begin{array}{lllllllllllllllllll}170 & 160 & 150 & 140 & 130 & 120 & 110 & 100 & 90 & 80 & 70 & 60 & 50 & 40 & 30 & 20 & 10 & 0\end{array}$

Figure S31. ${ }^{13} \mathrm{C}$ NMR spectrum of compound $\boldsymbol{r a c}-\mathbf{6}$ and $\boldsymbol{m e s}-\mathbf{6}\left(125 \mathrm{MHz}, \mathrm{C}_{2} \mathrm{D}_{2} \mathrm{Cl}_{4}, 373.2 \mathrm{~K}\right)$ 

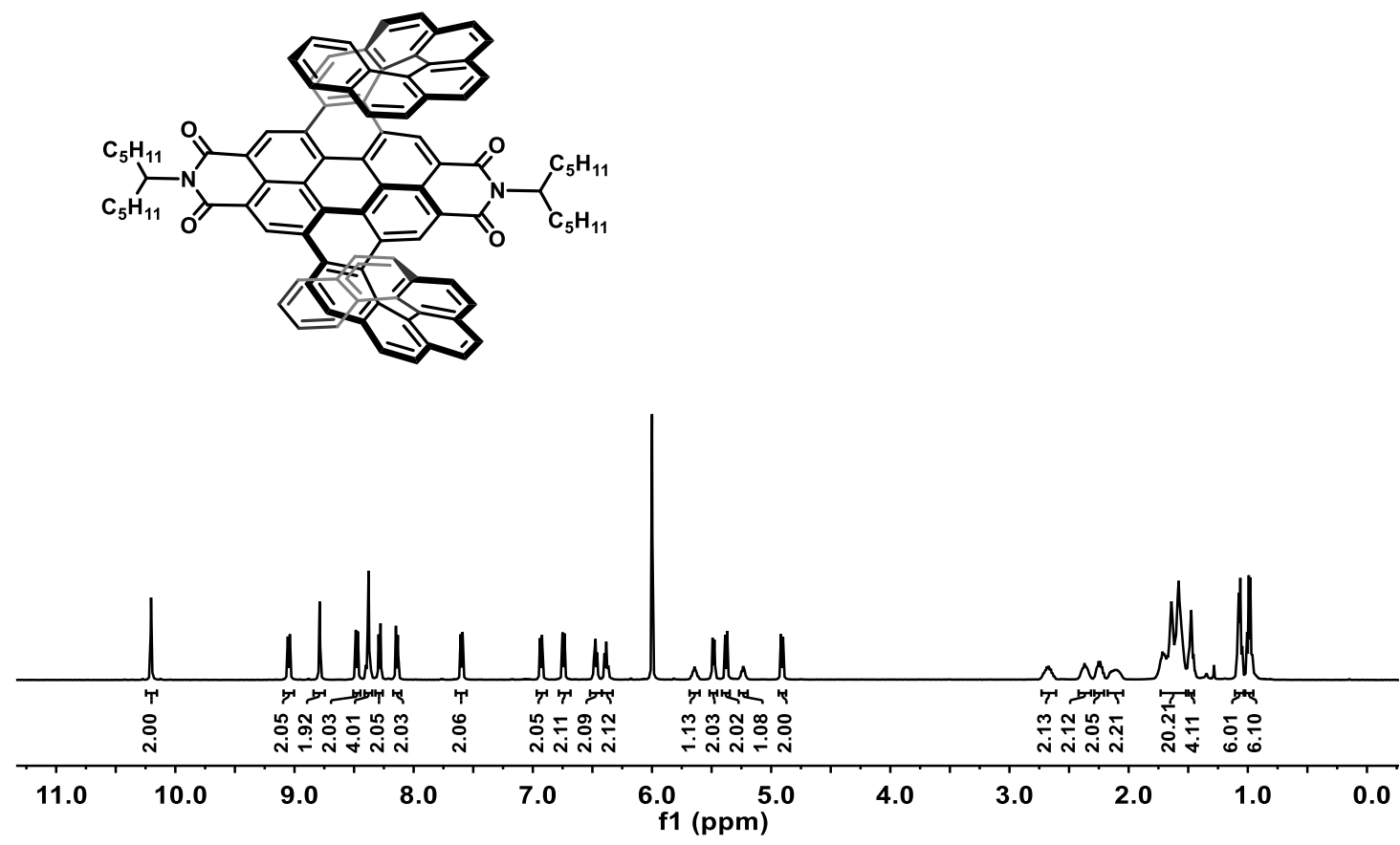

Figure S32. ${ }^{1} \mathrm{H}$ NMR spectrum of compound PD8H-6R (500 MHz, $\left.\mathrm{C}_{2} \mathrm{D}_{2} \mathrm{Cl}_{4}, 373.2 \mathrm{~K}\right)$

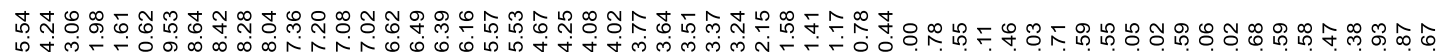

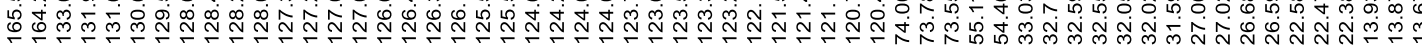

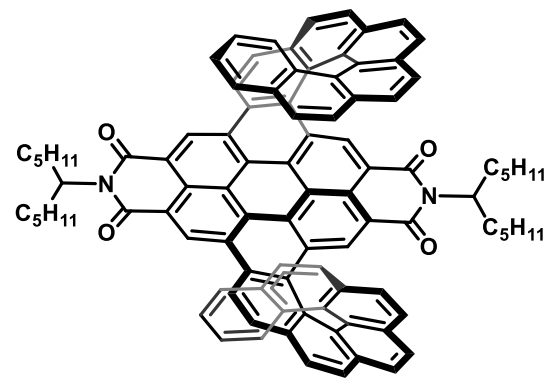

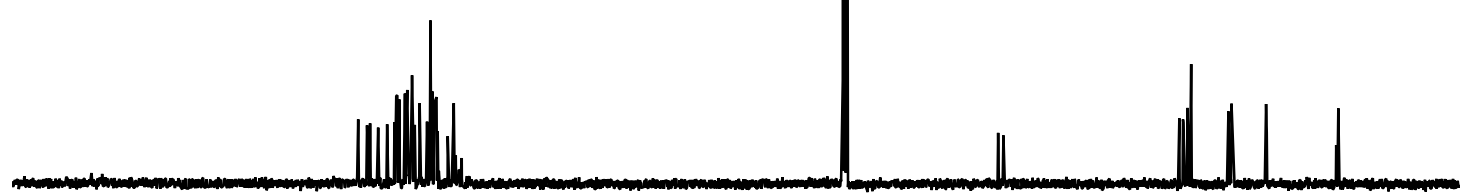

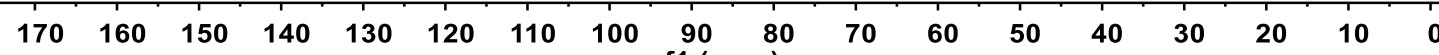
f1 (ppm)

Figure S33. ${ }^{13} \mathrm{C}$ NMR spectrum of compound PD8H-6R (125 MHz, $\left.\mathrm{C}_{2} \mathrm{D}_{2} \mathrm{Cl}_{4}, 373.2 \mathrm{~K}\right)$ 


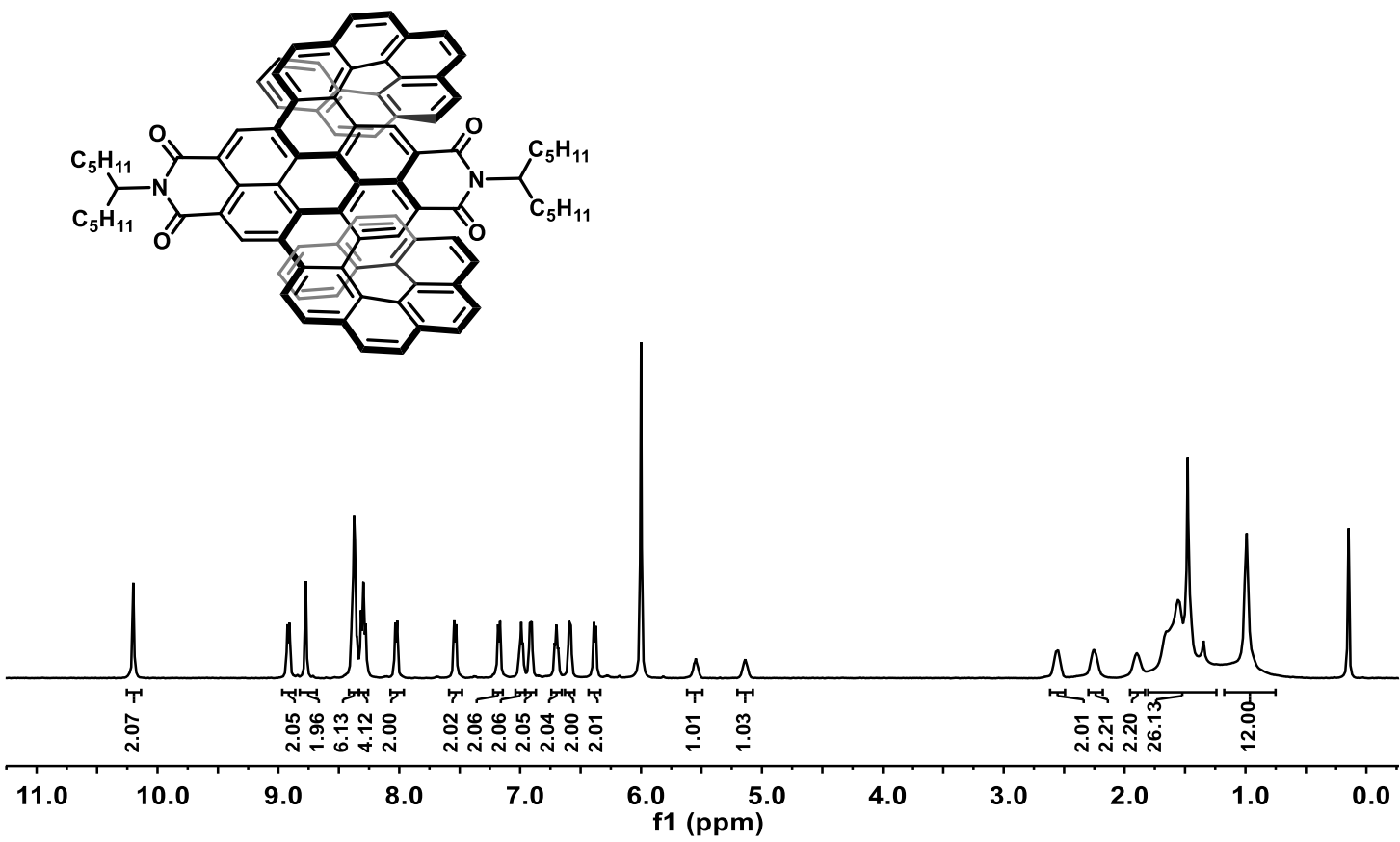

Figure S34. ${ }^{1} \mathrm{H}$ NMR spectrum of compound PD8H-6M (500 MHz, $\left.\mathrm{C}_{2} \mathrm{D}_{2} \mathrm{Cl}_{4}, 373.2 \mathrm{~K}\right)$

管

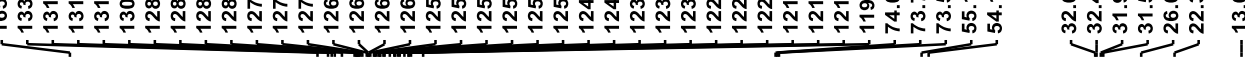
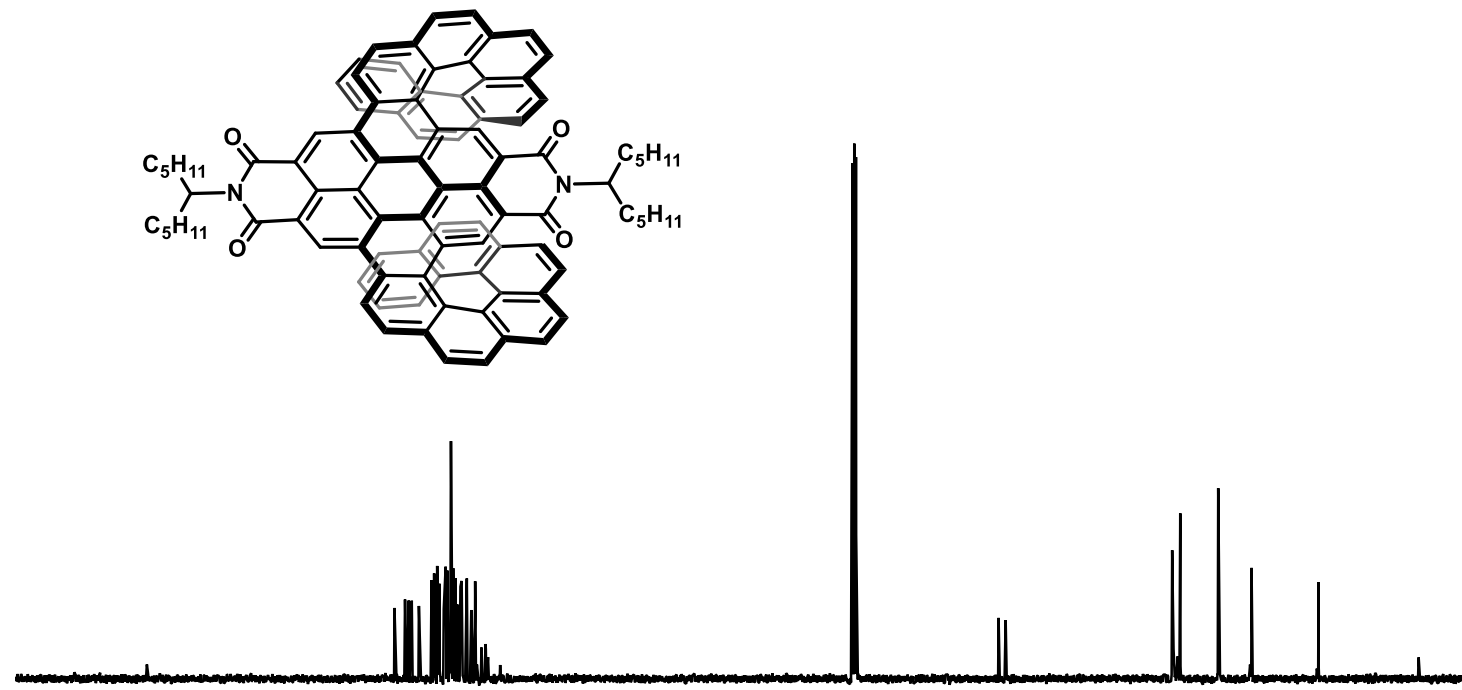

$\begin{array}{lllllllllllllllllll}180 & 170 & 160 & 150 & 140 & 130 & 120 & 110 & 100 \underset{f 1}{\stackrel{90}{(\mathrm{ppm})}} \mathbf{8 0} & 70 & 60 & 50 & 40 & 30 & 20 & 10 & 0\end{array}$

Figure S35. ${ }^{13} \mathrm{C}$ NMR spectrum of compound PD8H-6M (125 MHz, $\left.\mathrm{C}_{2} \mathrm{D}_{2} \mathrm{Cl}_{4}, 373.2 \mathrm{~K}\right)$ 


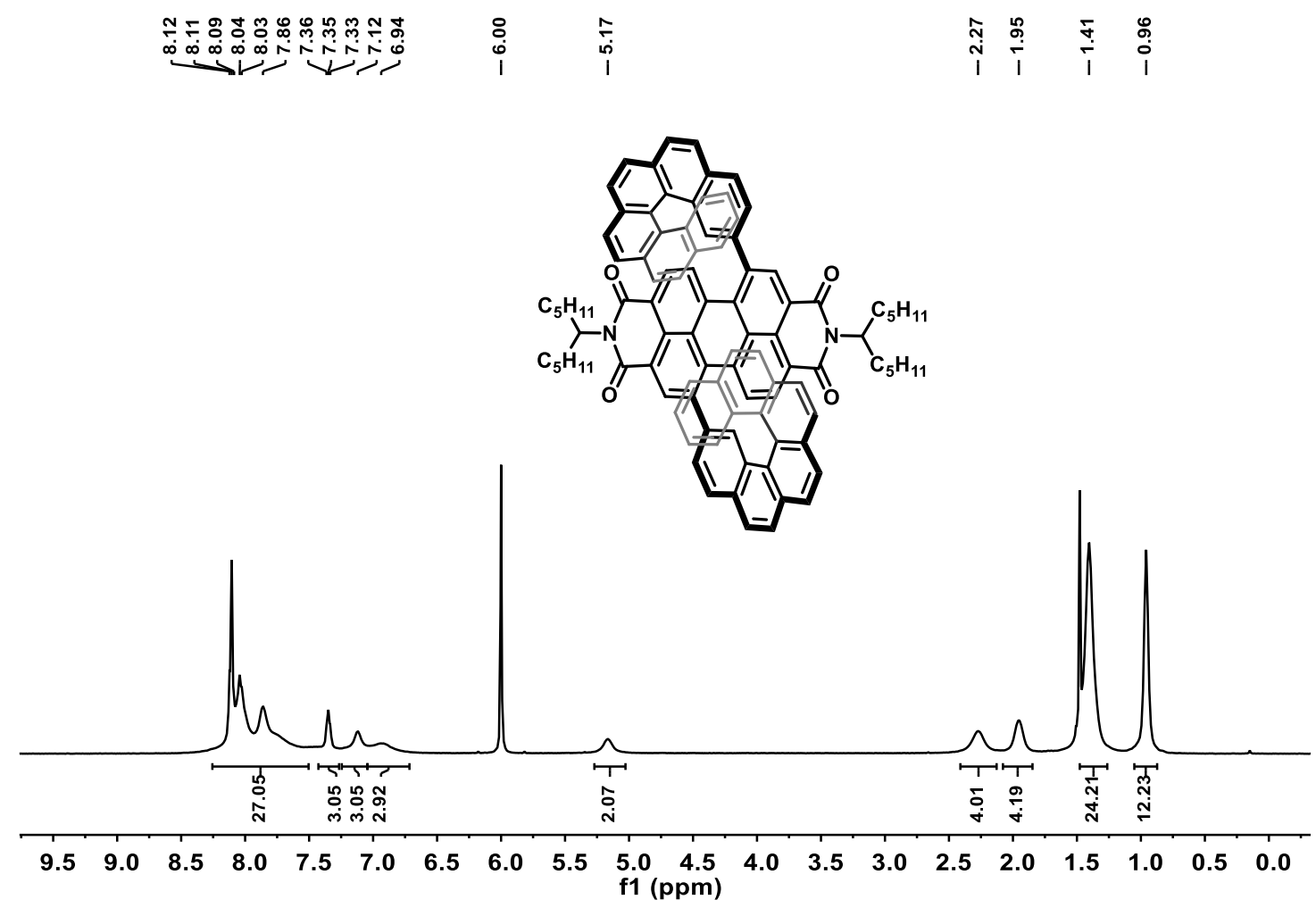

Figure S36. ${ }^{1} \mathrm{H}$ NMR spectrum of compound rac-7 $\left(500 \mathrm{MHz}, \mathrm{C}_{2} \mathrm{D}_{2} \mathrm{Cl}_{4}, 373.2 \mathrm{~K}\right)$
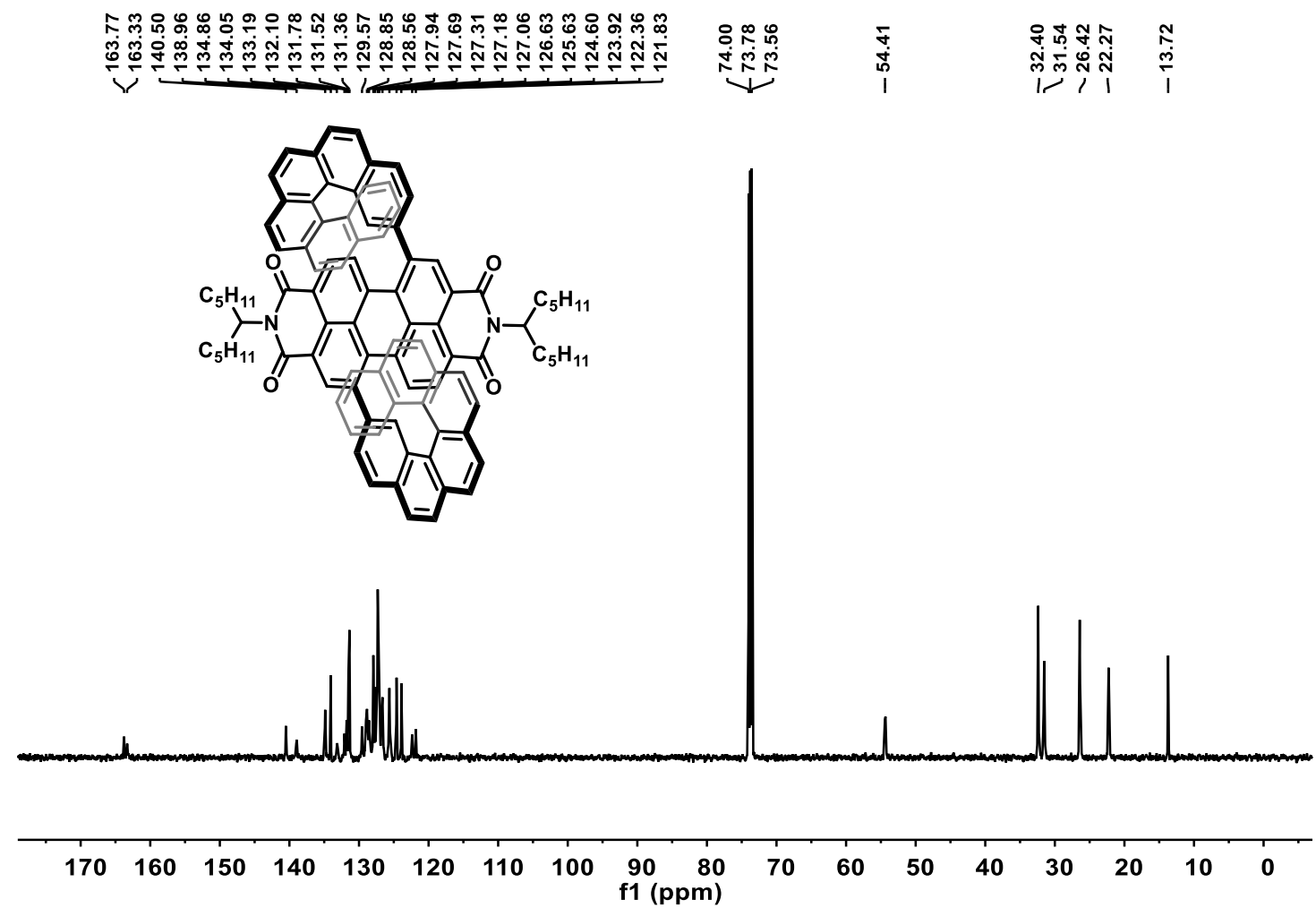

Figure S37. ${ }^{13} \mathrm{C}$ NMR spectrum of compound rac-7 (125 MHz, $\left.\mathrm{C}_{2} \mathrm{D}_{2} \mathrm{Cl}_{4}, 373.2 \mathrm{~K}\right)$ 


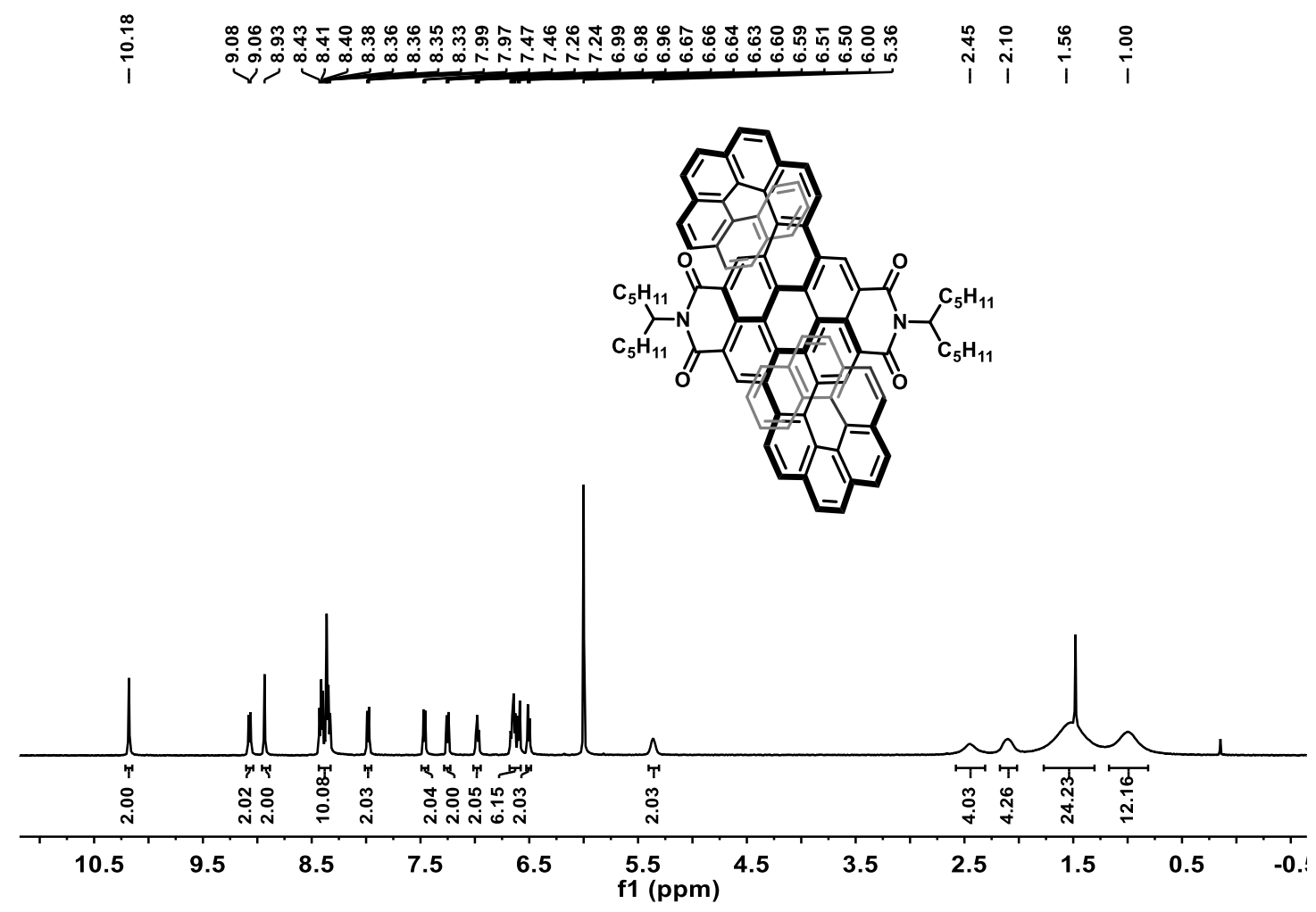

Figure S38. ${ }^{1} \mathrm{H}$ NMR spectrum of compound PD8H-7R (500 MHz, $\left.\mathrm{C}_{2} \mathrm{D}_{2} \mathrm{Cl}_{4}, 373.2 \mathrm{~K}\right)$
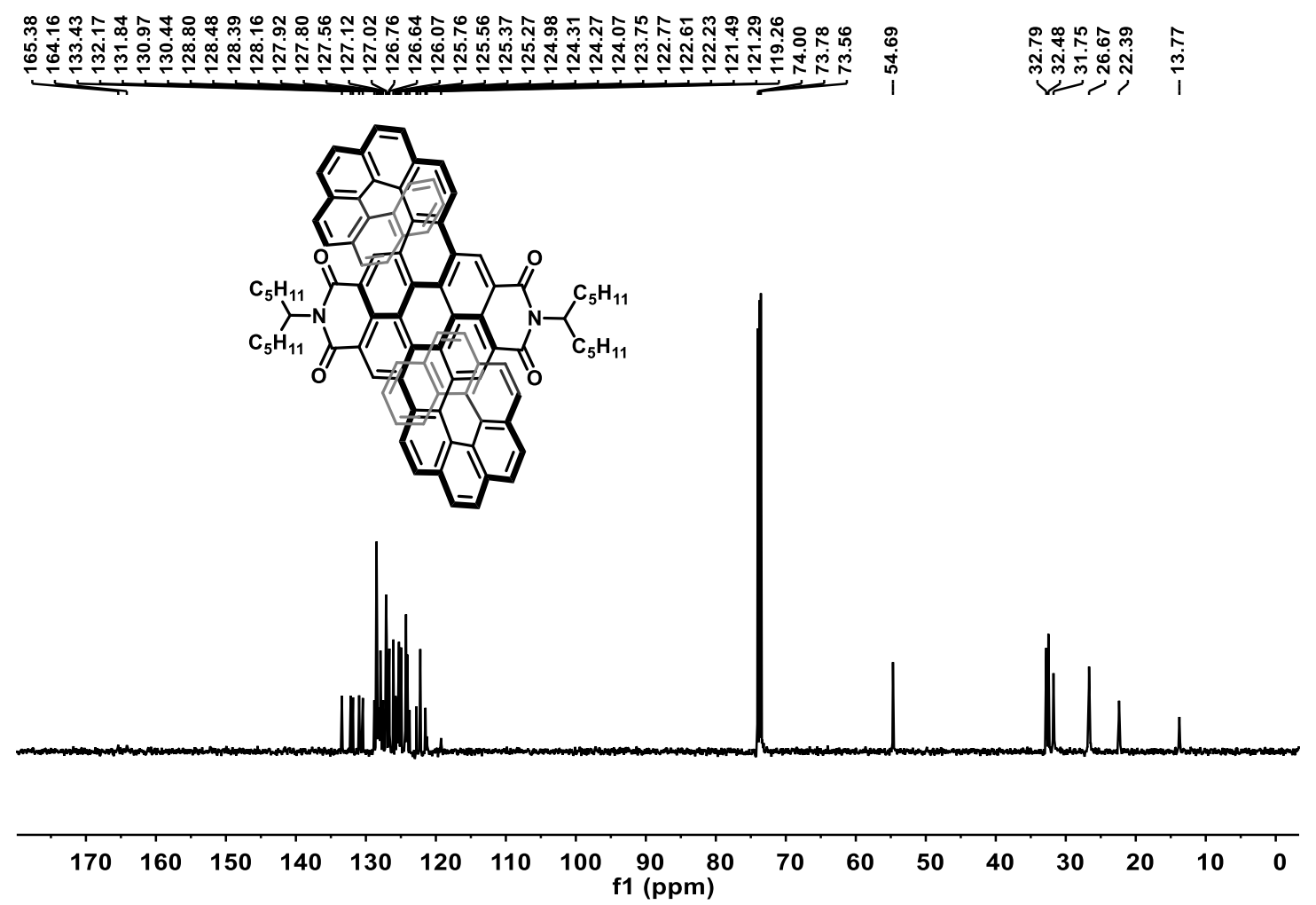

Figure S39. ${ }^{13} \mathrm{C}$ NMR spectrum of compound PD8H-7R (125 MHz, $\left.\mathrm{C}_{2} \mathrm{D}_{2} \mathrm{Cl}_{4}, 373.2 \mathrm{~K}\right)$ 


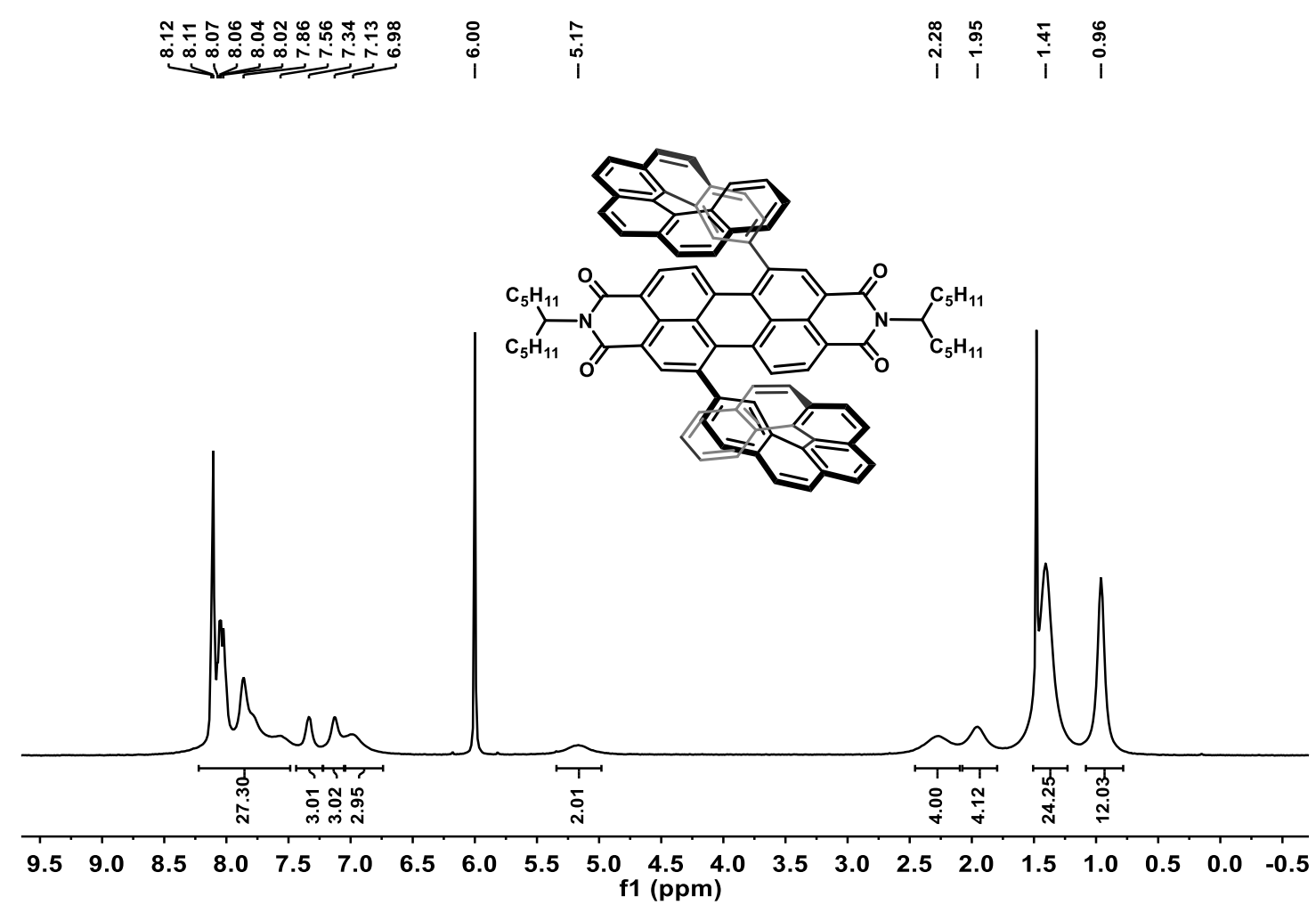

Figure S40. ${ }^{1} \mathrm{H}$ NMR spectrum of compound $\boldsymbol{m e s}-7\left(500 \mathrm{MHz}, \mathrm{C}_{2} \mathrm{D}_{2} \mathrm{Cl}_{4}, 373.2 \mathrm{~K}\right)$
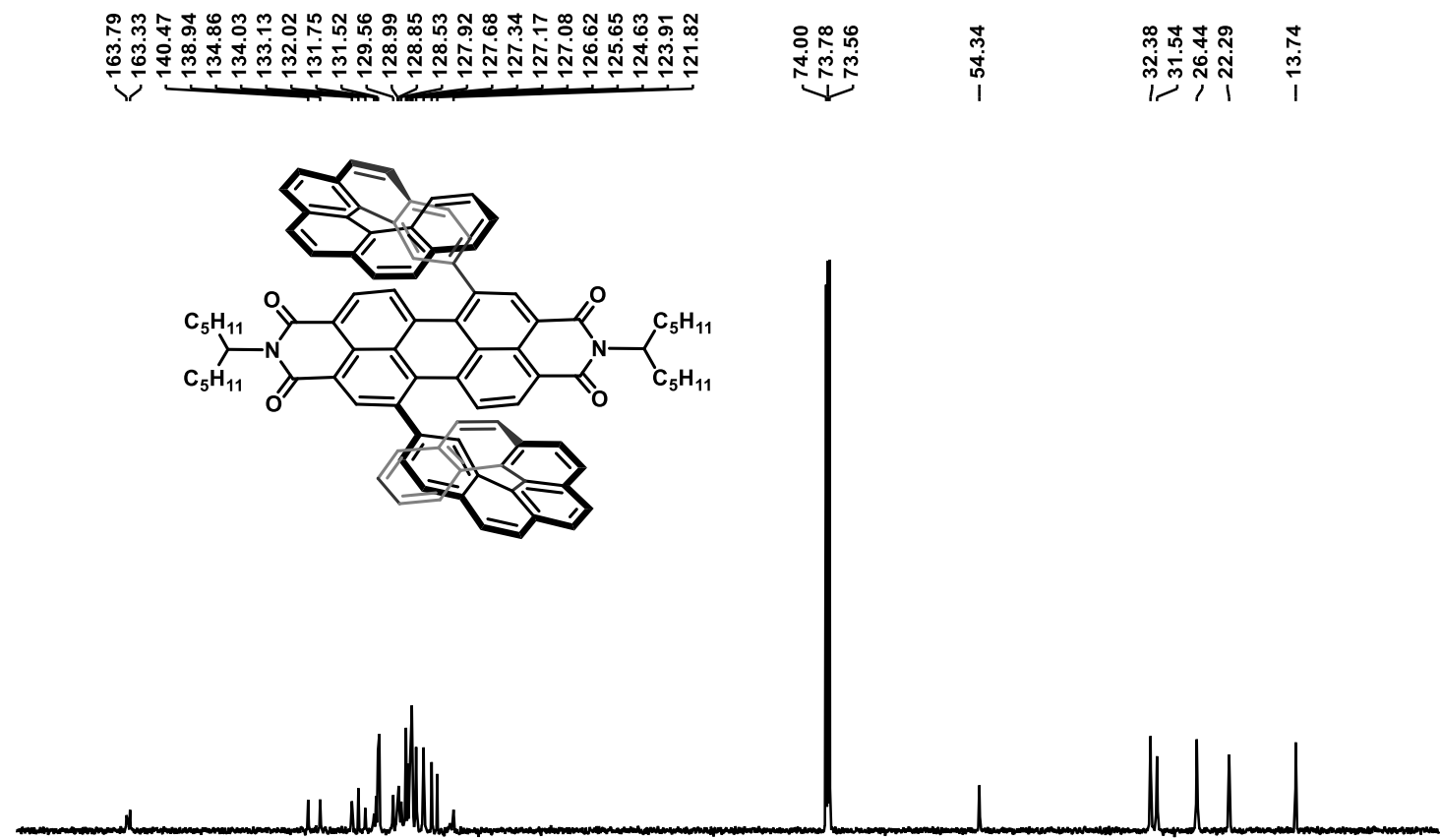

$\begin{array}{llllllllll}170 & 160 & 150 & 140 & 130 & 120 & 110 & 100 & 90 & 80 \\ \text { f1 (ppm) }\end{array}$

Figure S41. ${ }^{13} \mathrm{C}$ NMR spectrum of compound mes-7 (125 MHz, $\left.\mathrm{C}_{2} \mathrm{D}_{2} \mathrm{Cl}_{4}, 373.2 \mathrm{~K}\right)$ 


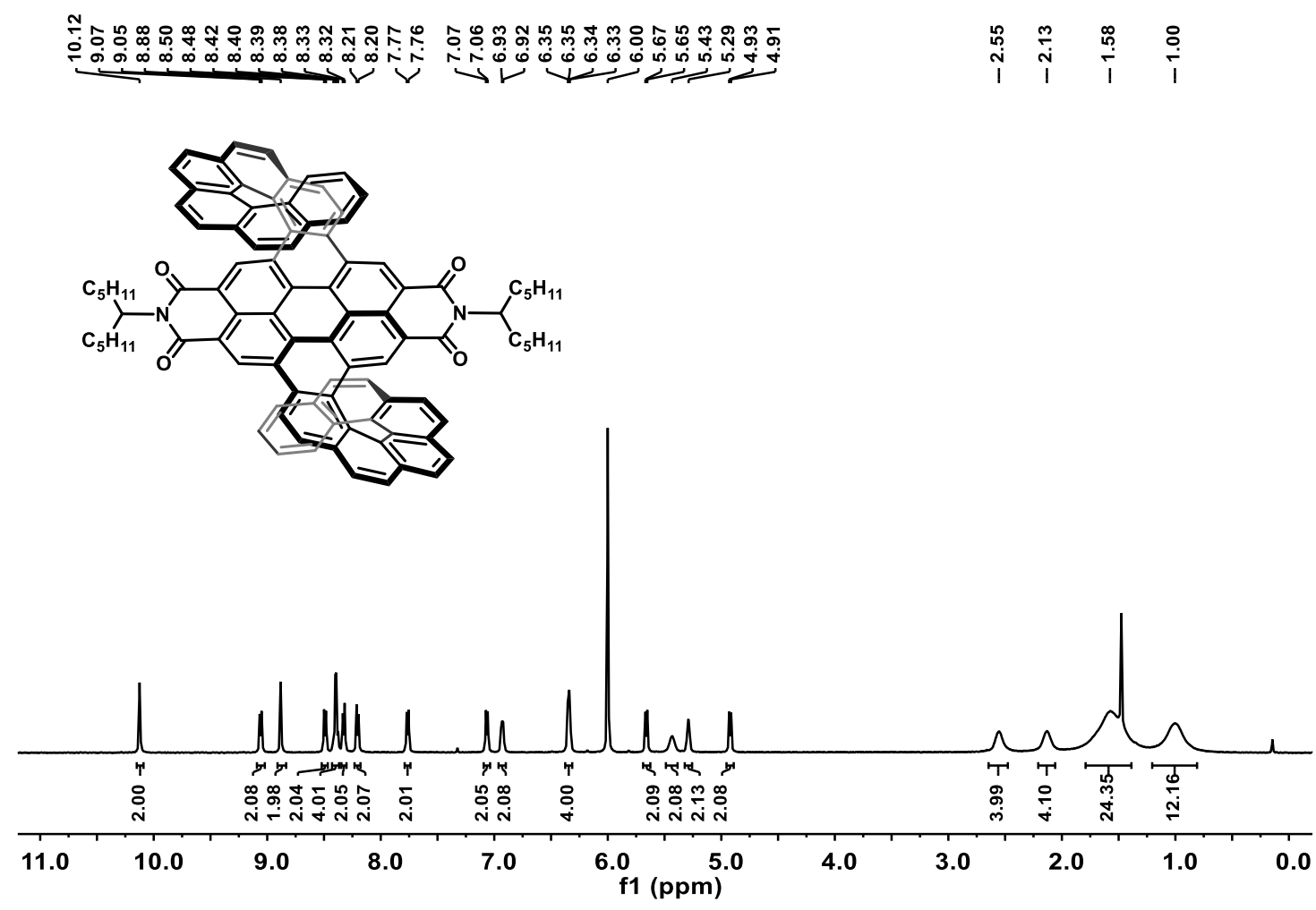

Figure S42. ${ }^{1} \mathrm{H}$ NMR spectrum of compound PD8H-7M (500 MHz, $\left.\mathrm{C}_{2} \mathrm{D}_{2} \mathrm{Cl}_{4}, 373.2 \mathrm{~K}\right)$
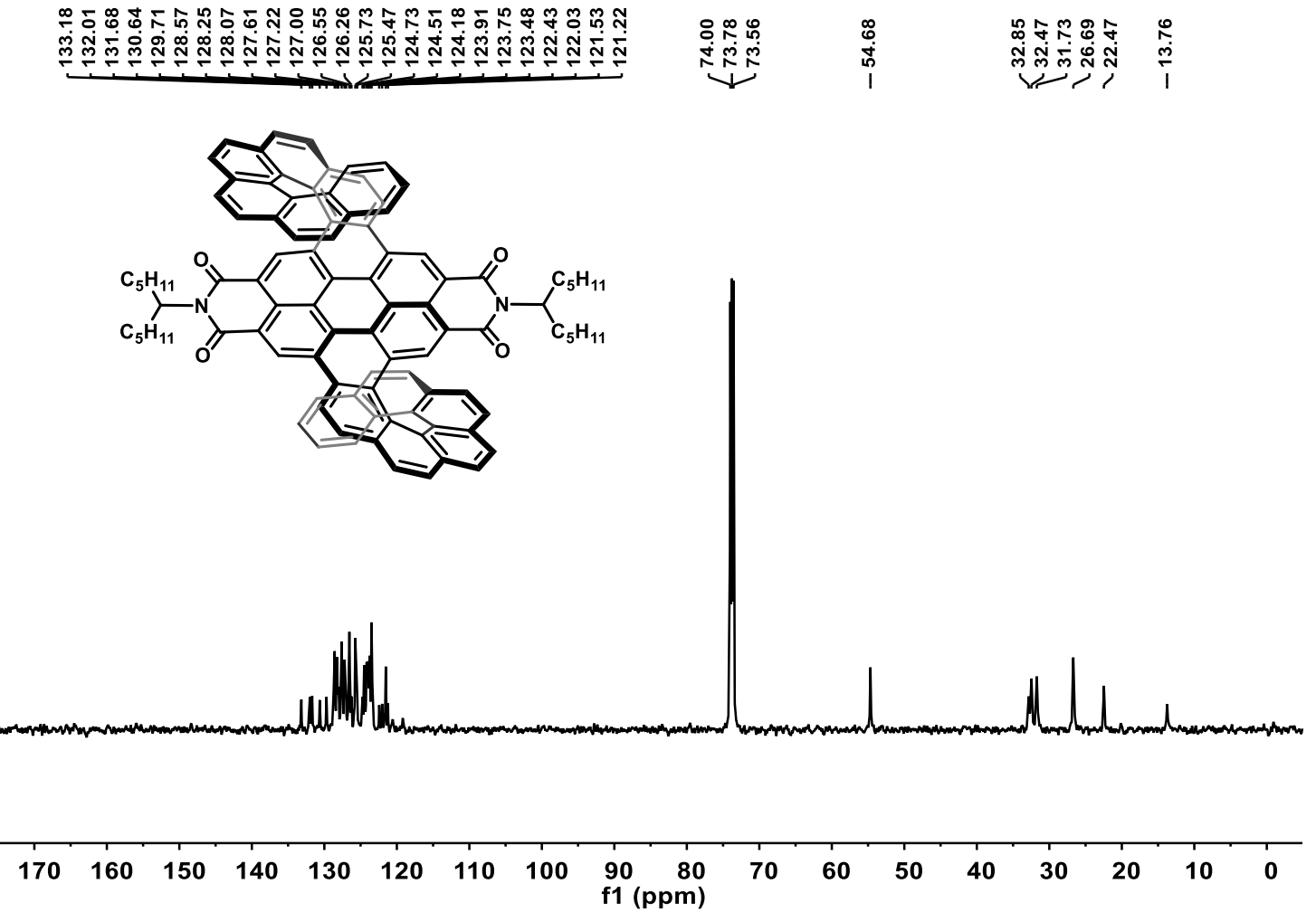

Figure S43. ${ }^{13} \mathrm{C}$ NMR spectrum of compound PD8H-7M (125 MHz, $\left.\mathrm{C}_{2} \mathrm{D}_{2} \mathrm{Cl}_{4}, 373.2 \mathrm{~K}\right)$ 


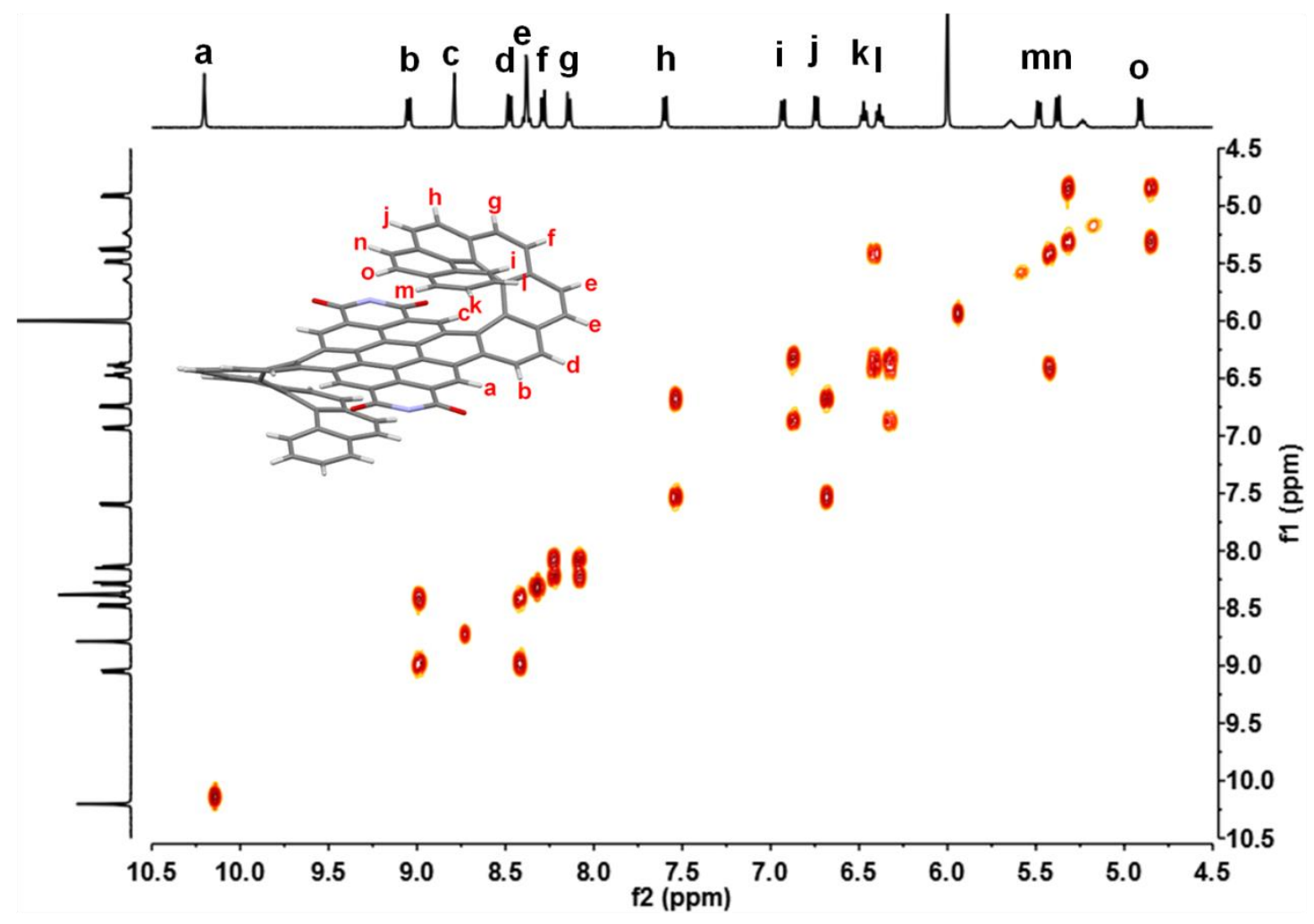

Figure S44. 2D ${ }^{1} \mathrm{H}-{ }^{1} \mathrm{H}$ COSY NMR spectrum of PD8H-6R (500 MHz, $\mathrm{C}_{2} \mathrm{D}_{2} \mathrm{Cl}_{4}, 373.2 \mathrm{~K}$ )

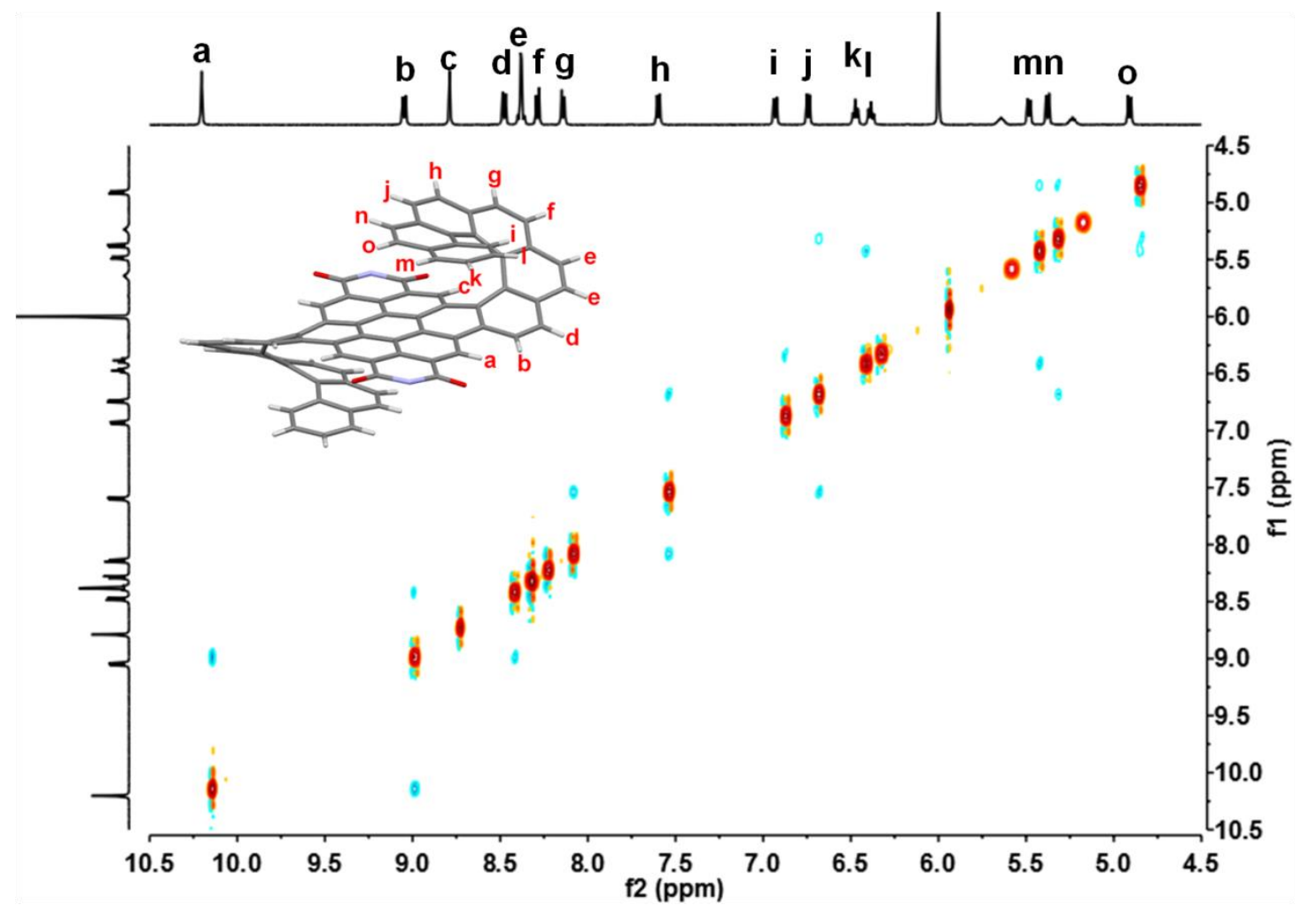

Figure S45. 2D ${ }^{1} \mathrm{H}-{ }^{1} \mathrm{H}$ NOESY NMR spectrum of PD8H-6R (500 MHz, $\left.\mathrm{C}_{2} \mathrm{D}_{2} \mathrm{Cl}_{4}, 373.2 \mathrm{~K}\right)$ 


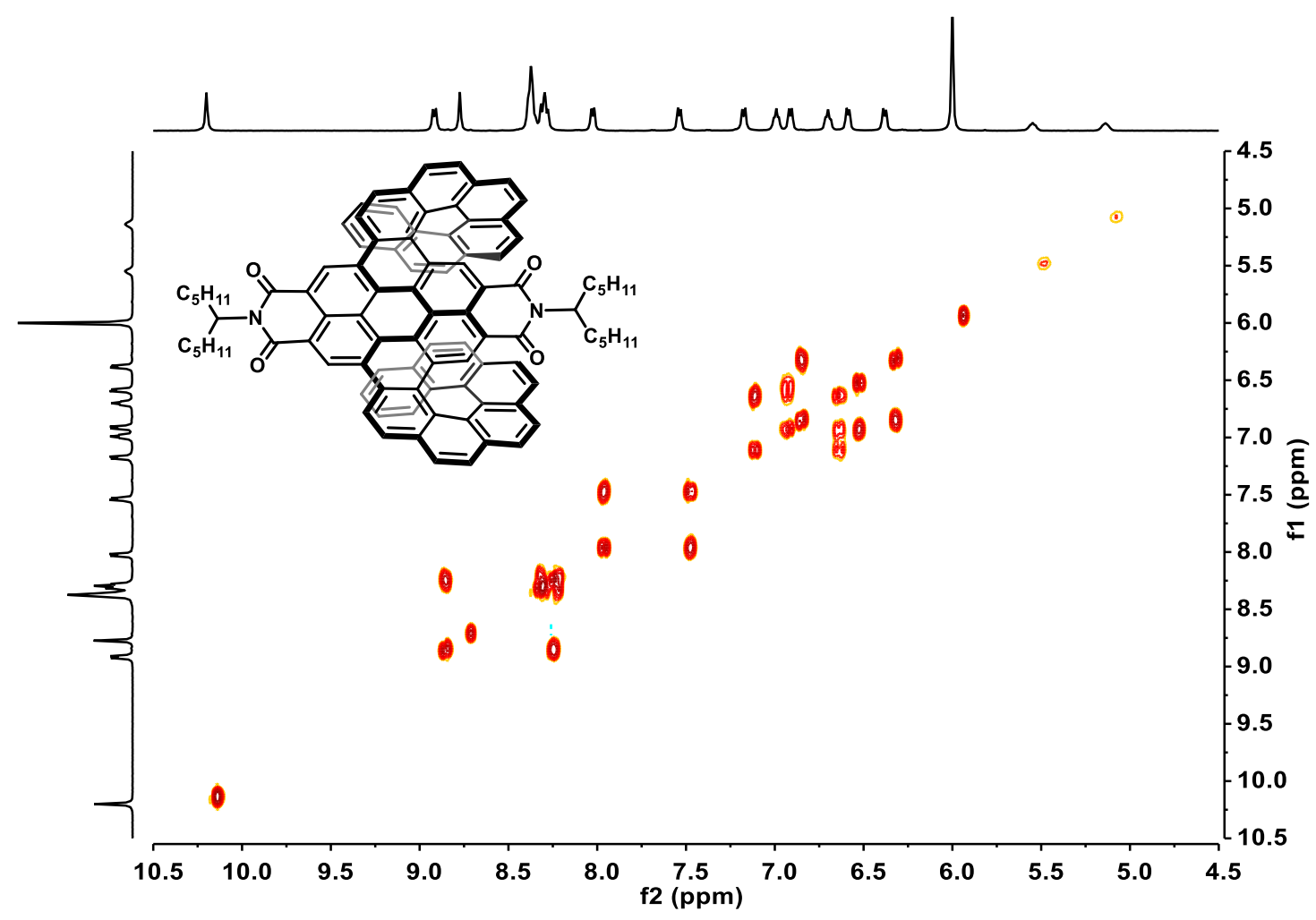

Figure S46. $2 \mathrm{D}{ }^{1} \mathrm{H}^{-}{ }^{-} \mathrm{H}$ COSY NMR spectrum of PD8H-6M $\left(500 \mathrm{MHz}, \mathrm{C}_{2} \mathrm{D}_{2} \mathrm{Cl}_{4}, 373.2 \mathrm{~K}\right)$

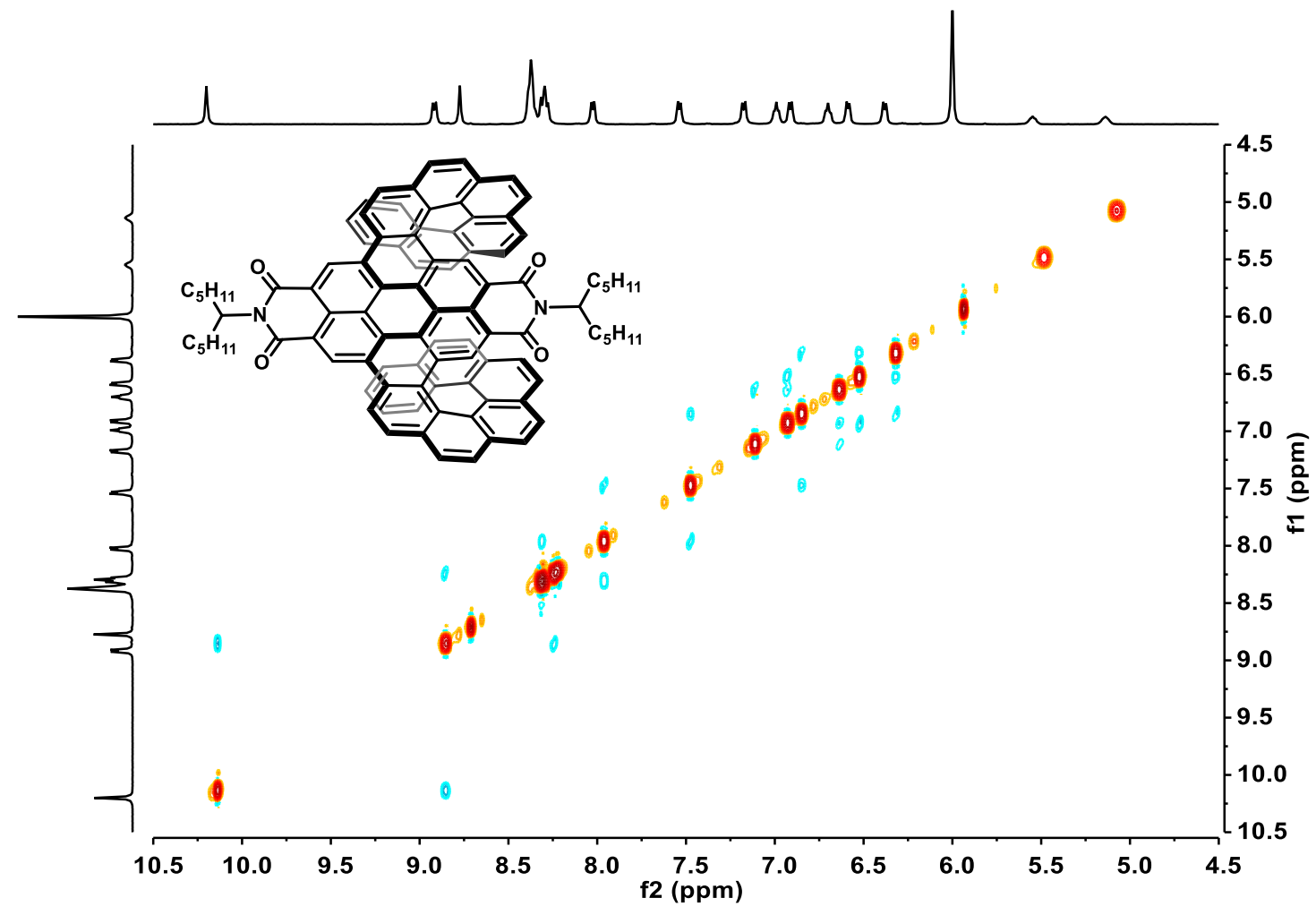

Figure S47. 2D ${ }^{1} \mathrm{H}-{ }^{1} \mathrm{H}$ NOESY NMR spectrum of PD8H-6M (500 MHz, $\left.\mathrm{C}_{2} \mathrm{D}_{2} \mathrm{Cl}_{4}, 373.2 \mathrm{~K}\right)$ 


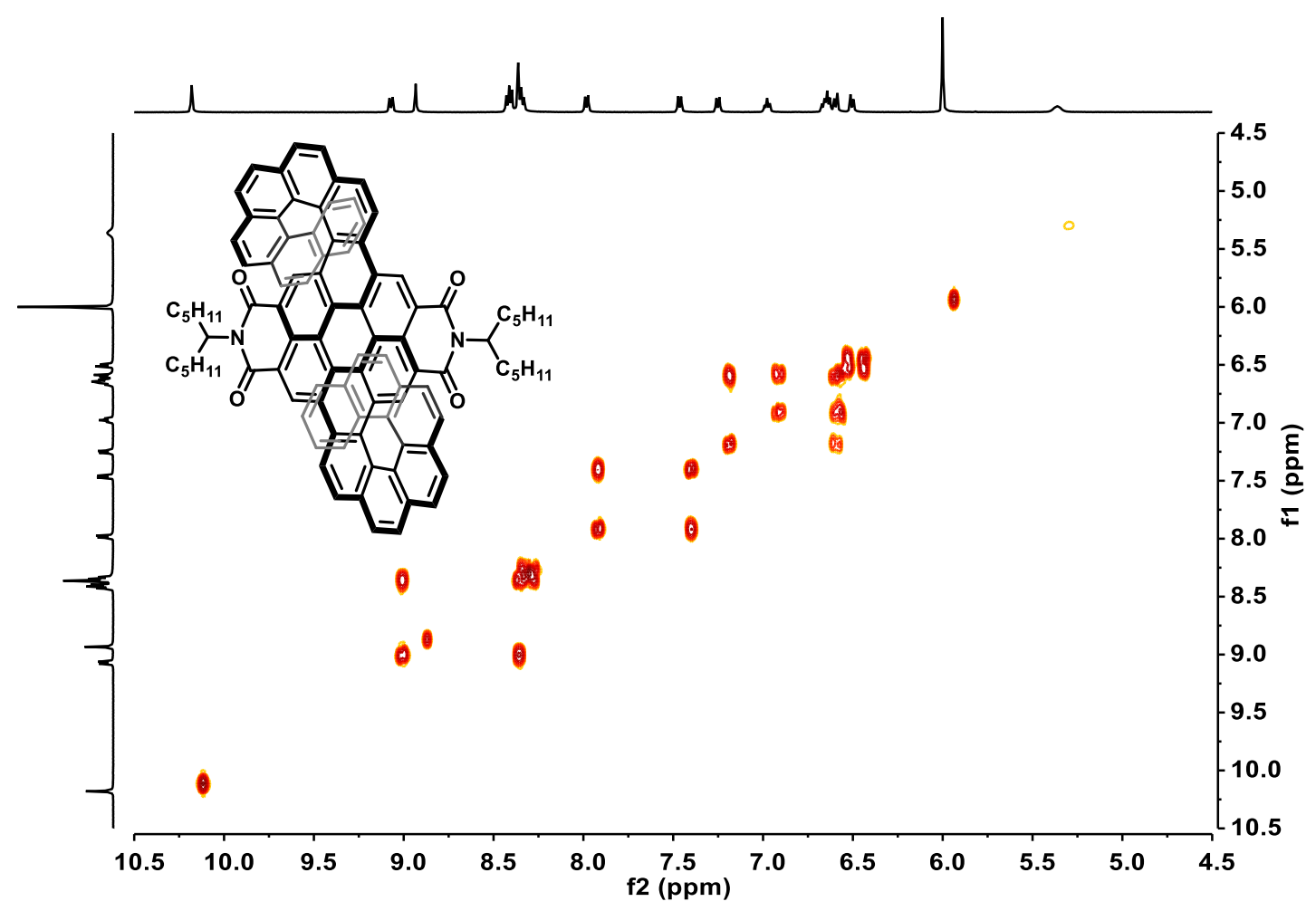

Figure S48. 2D ${ }^{1} \mathrm{H}^{-1} \mathrm{H}$ COSY NMR spectrum of PD8H-7R (500 MHz, $\mathrm{C}_{2} \mathrm{D}_{2} \mathrm{Cl}_{4}, 373.2 \mathrm{~K}$ )

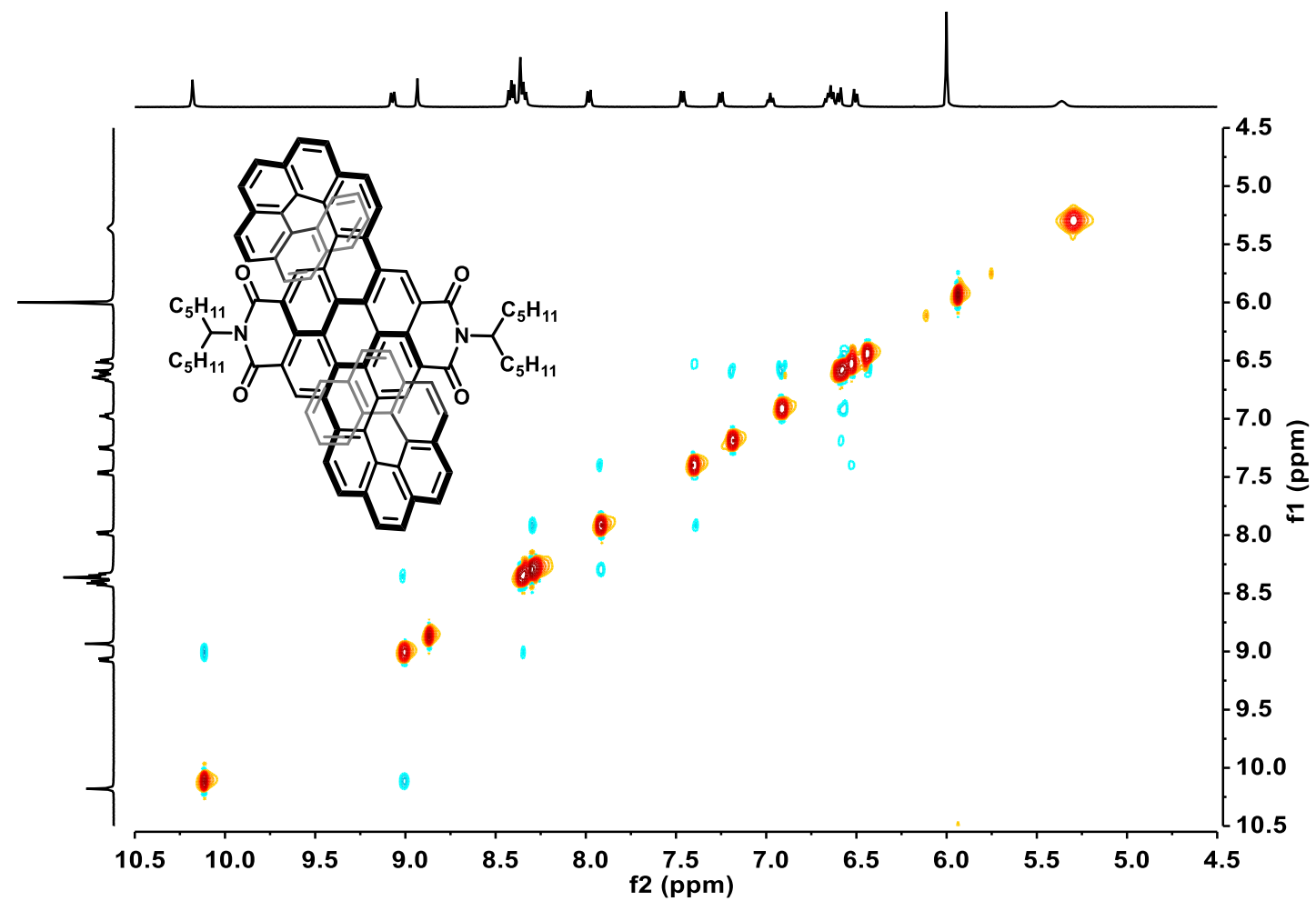

Figure S49. 2D ${ }^{1} \mathrm{H}-{ }^{1} \mathrm{H}$ NOESY NMR spectrum of PD8H-7R (500 MHz, $\left.\mathrm{C}_{2} \mathrm{D}_{2} \mathrm{Cl}_{4}, 373.2 \mathrm{~K}\right)$ 


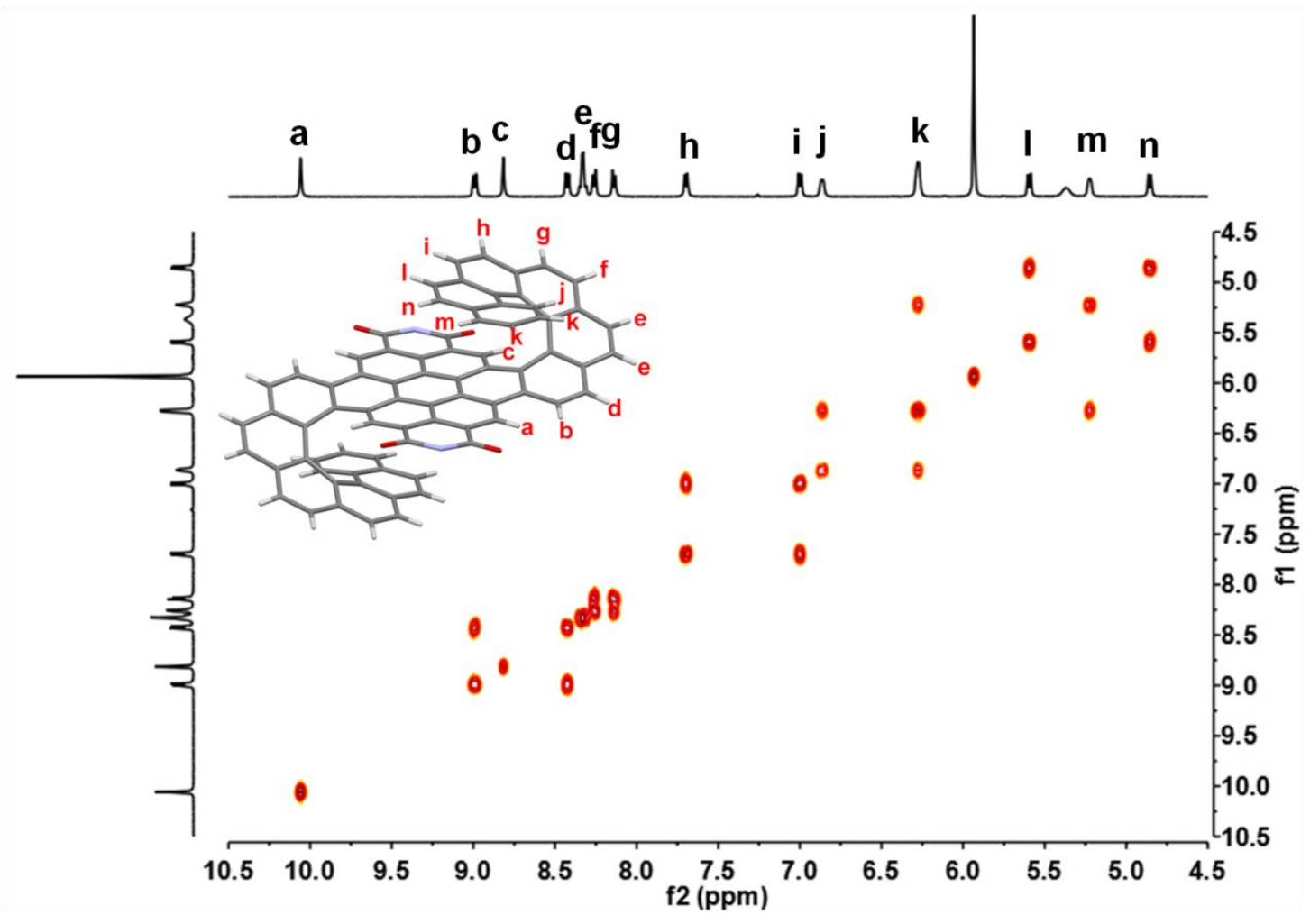

Figure S50. 2D ${ }^{1} \mathrm{H}-{ }^{1} \mathrm{H}$ COSY NMR spectrum of PD8H-7M $\left(500 \mathrm{MHz}, \mathrm{C}_{2} \mathrm{D}_{2} \mathrm{Cl}_{4}, 373.2 \mathrm{~K}\right)$

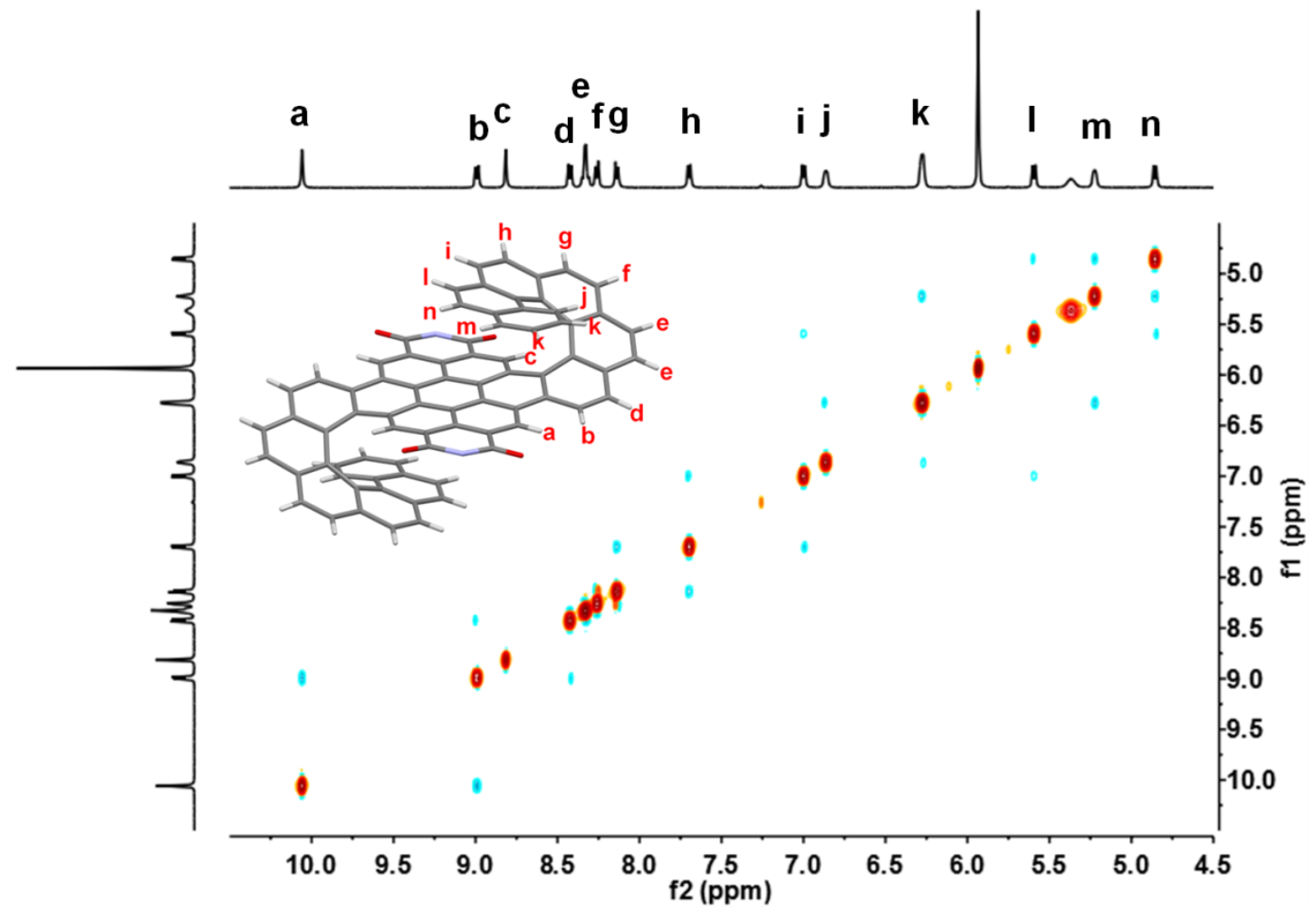

Figure S51. 2D ${ }^{1} \mathrm{H}-{ }^{1} \mathrm{H}$ NOESY NMR spectrum of compound PD8H-7M $\left(500 \mathrm{MHz}, \mathrm{C}_{2} \mathrm{D}_{2} \mathrm{Cl}_{4}\right.$, $373.2 \mathrm{~K})$ 


\section{HRMS}
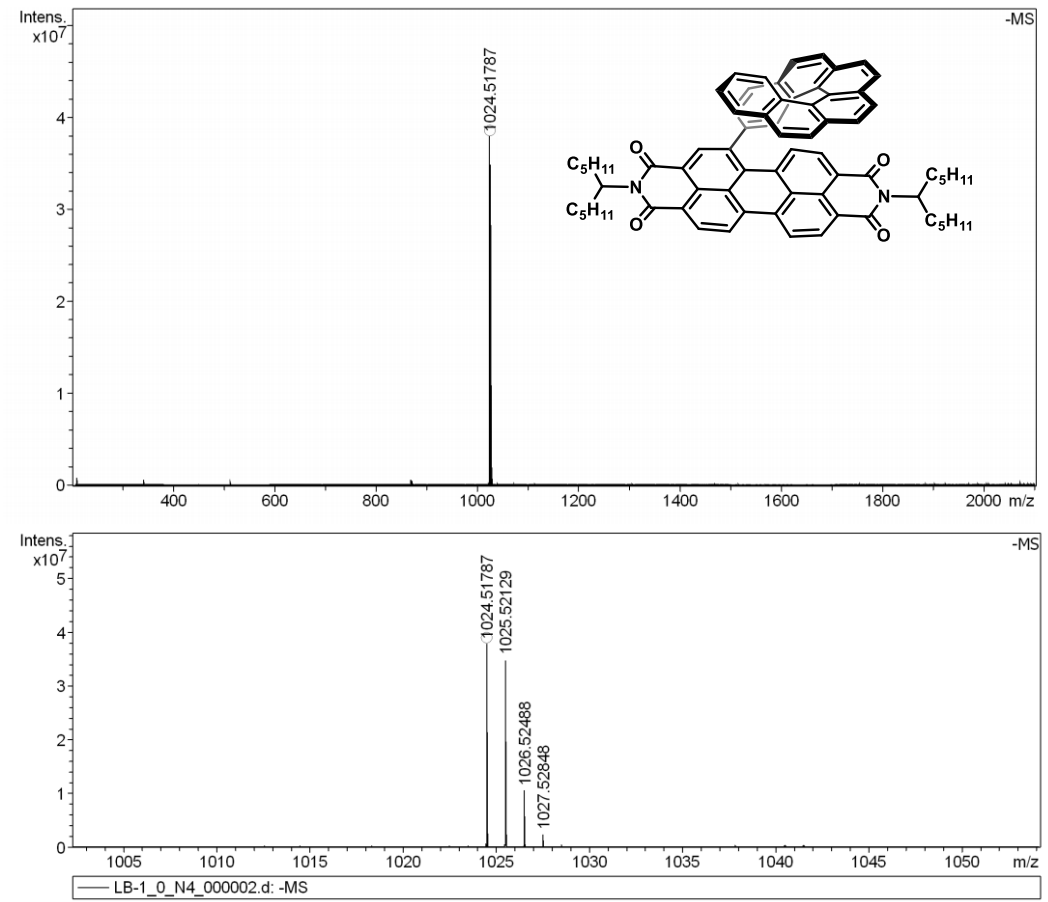

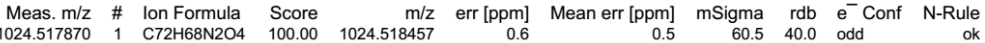

Figure S52. High-resolution mass spectrum (HRMS) of compound rac-5
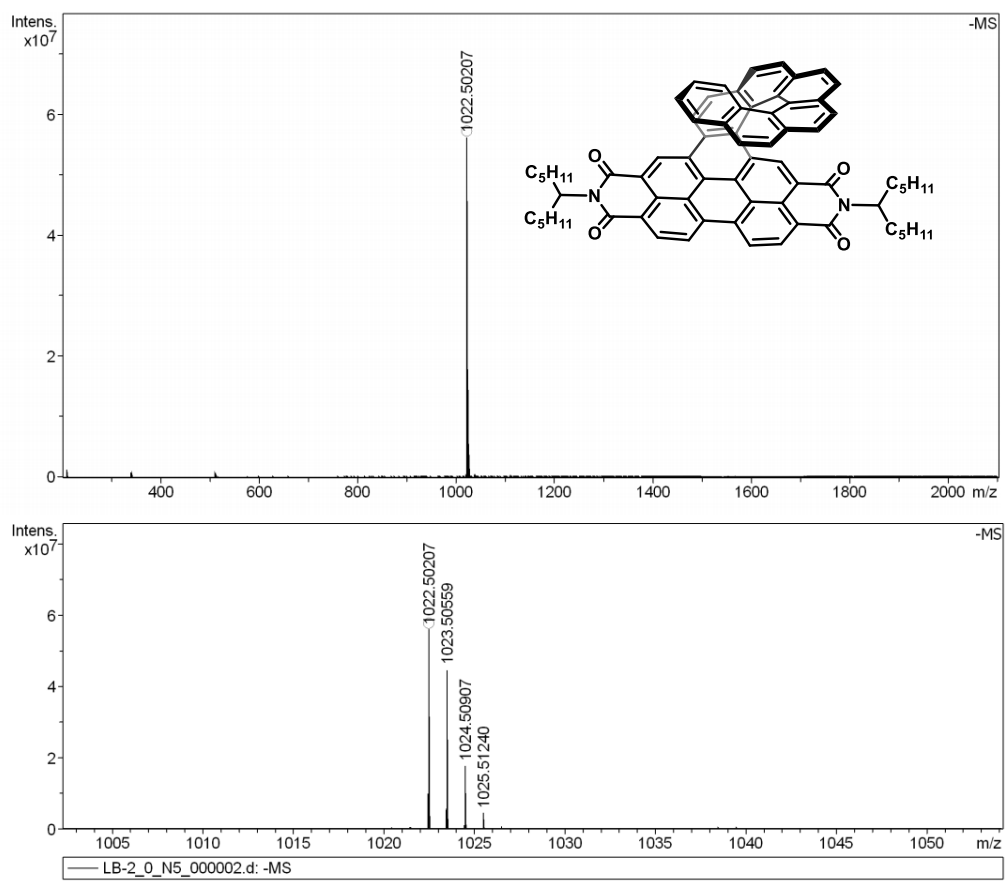

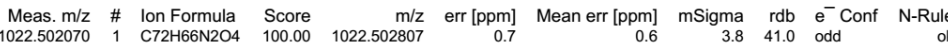

Figure S53. High-resolution mass spectrum (HRMS) of compound P8H 

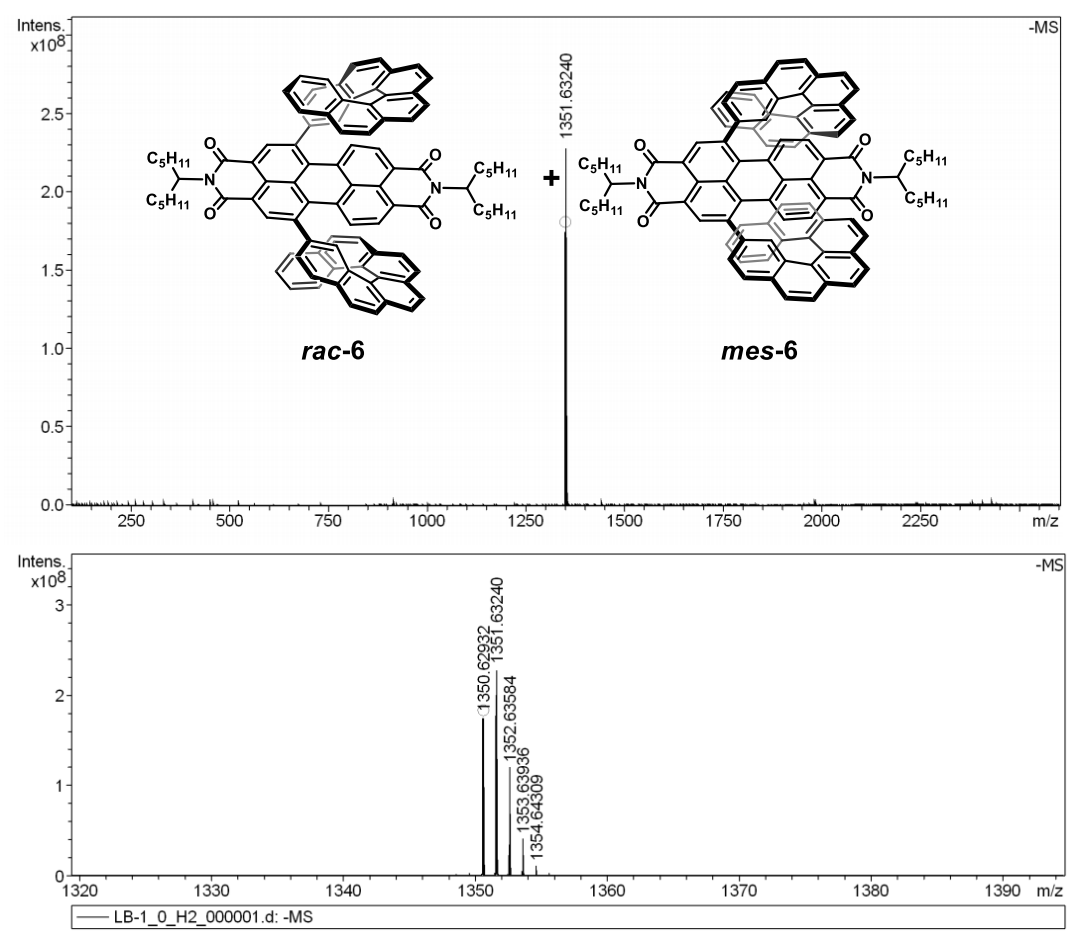

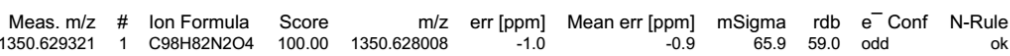

Figure S54. High-resolution mass spectra (HRMS) of compound rac-6 and mes-6
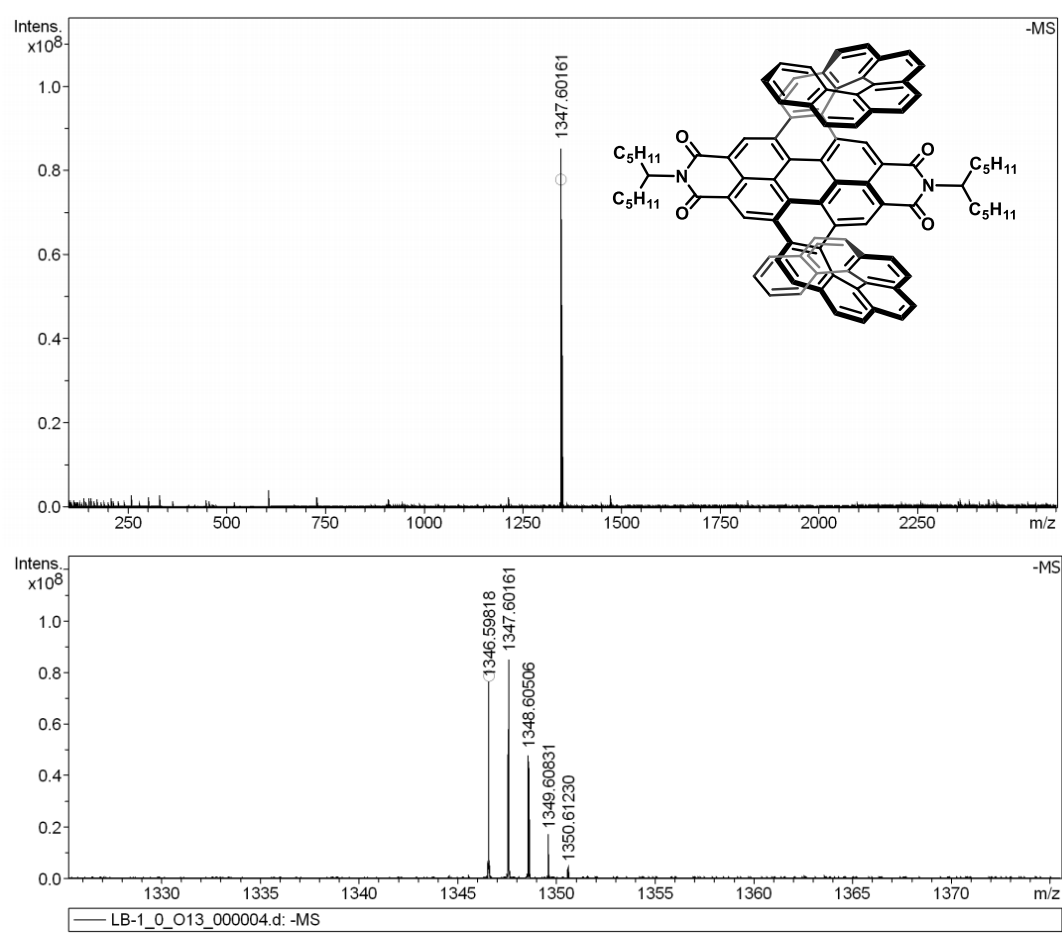

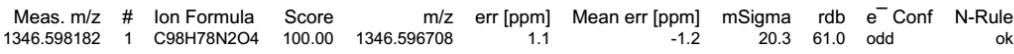

Figure S55. High-resolution mass spectra (HRMS) of compound PD8H-6R 

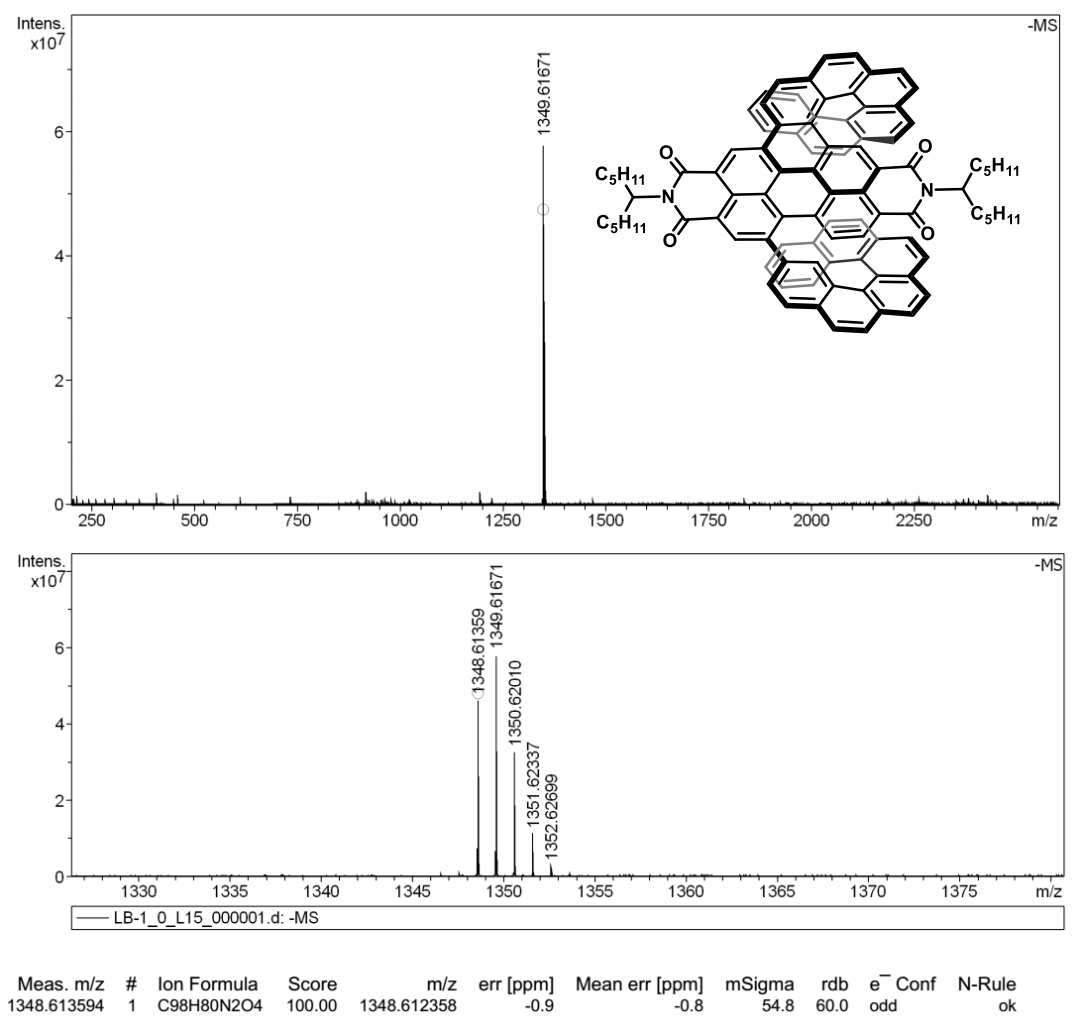

Figure S56. High-resolution mass spectra (HRMS) of the partially annulated intermediate of

\section{PD8H-6M}
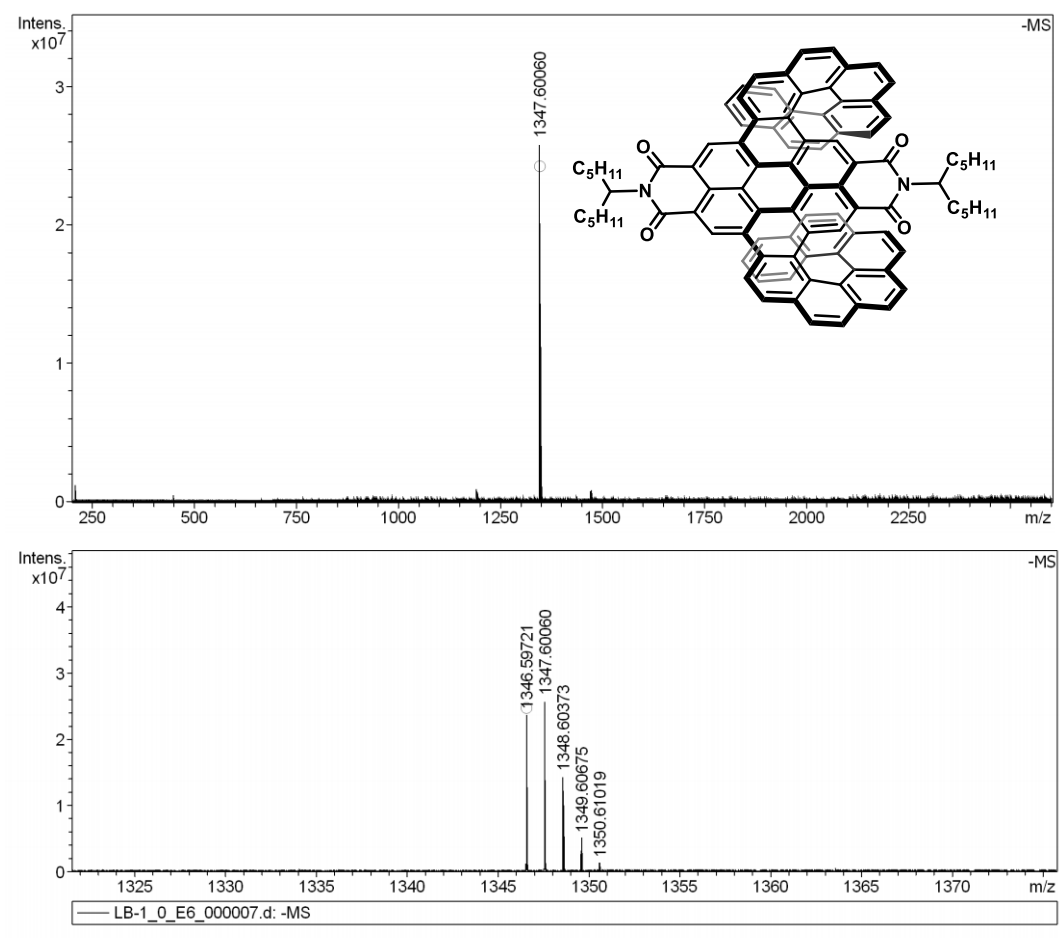

Meas. $\mathrm{m} / \mathrm{z}$ \# $\quad$ lon Formula Score $\mathrm{m} / \mathrm{z}$ err [ppm] Mean err [ppm] mSigma $\mathrm{rdb} \quad \mathrm{e}^{-}$Conf $\mathrm{N}-$ Rule

Figure S57. High-resolution mass spectra (HRMS) of compound PD8H-6M 

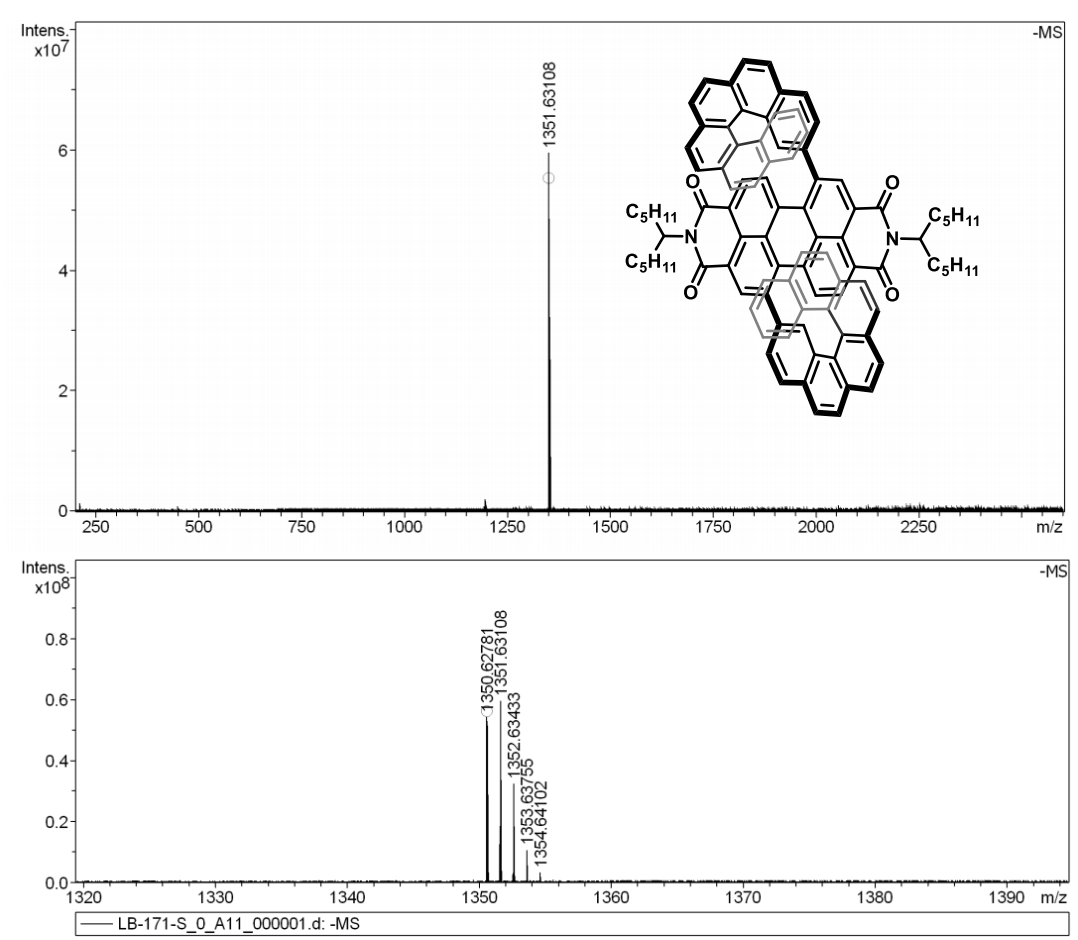

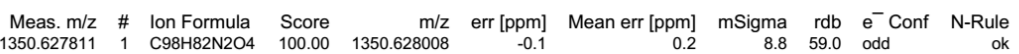

Figure S58. High-resolution mass spectra (HRMS) of compound rac-7
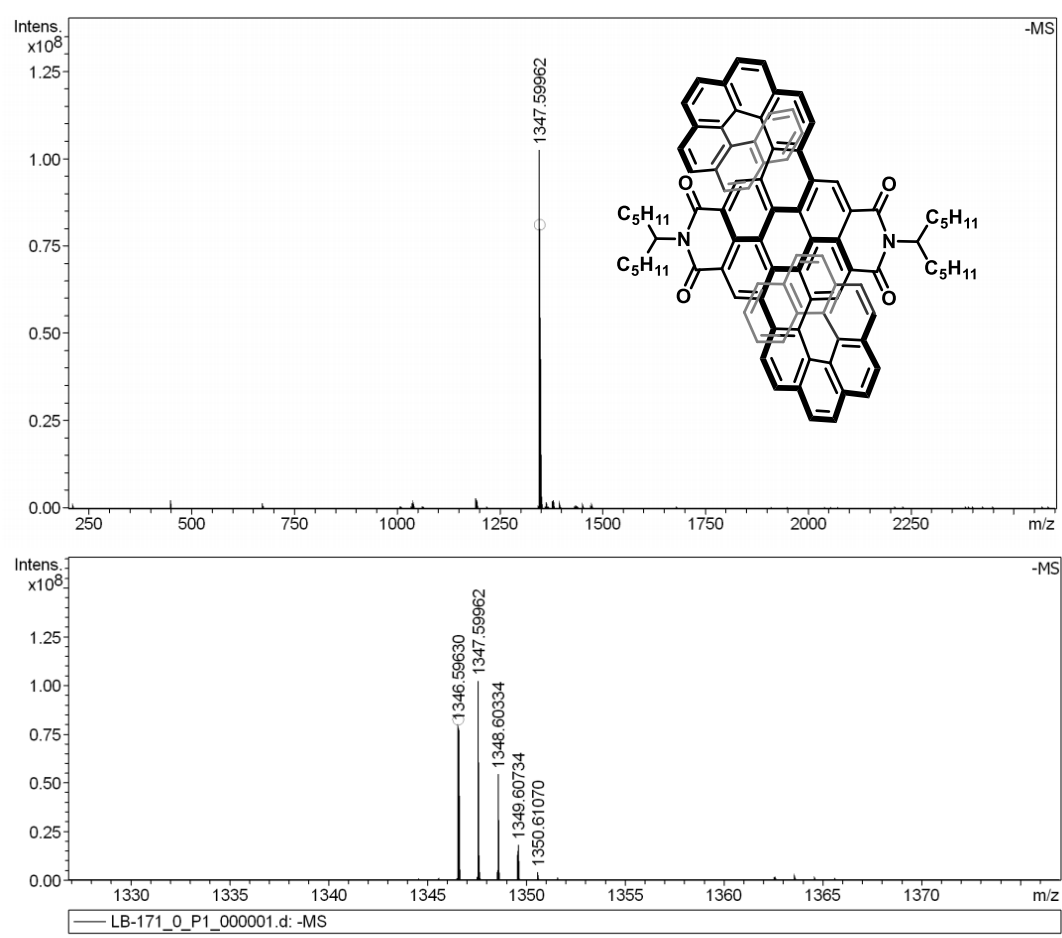

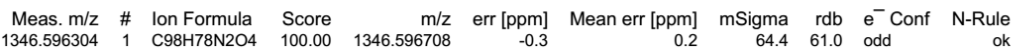

Figure S59. High-resolution mass spectra (HRMS) of compound PD8H-7R 

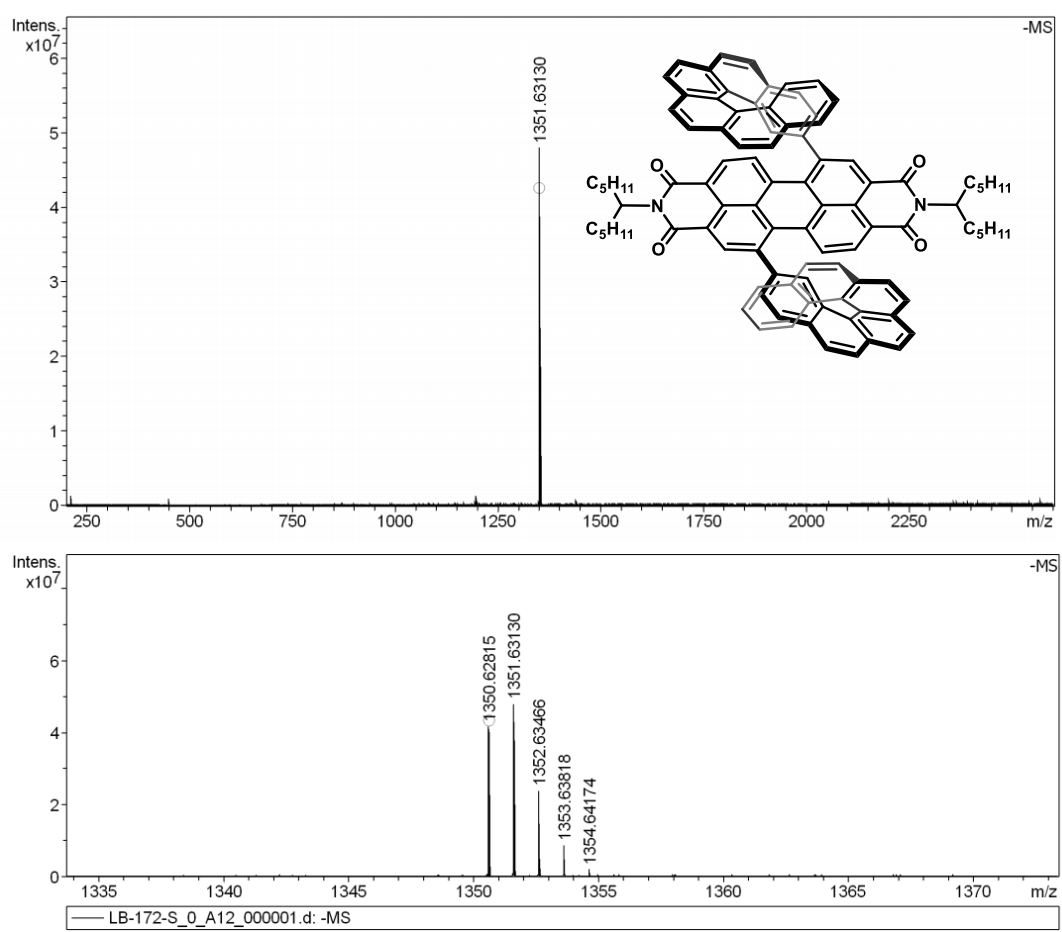

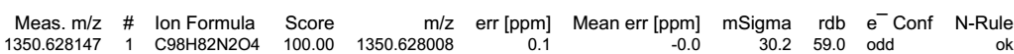

Figure S60. High-resolution mass spectra (HRMS) of compound mes-7
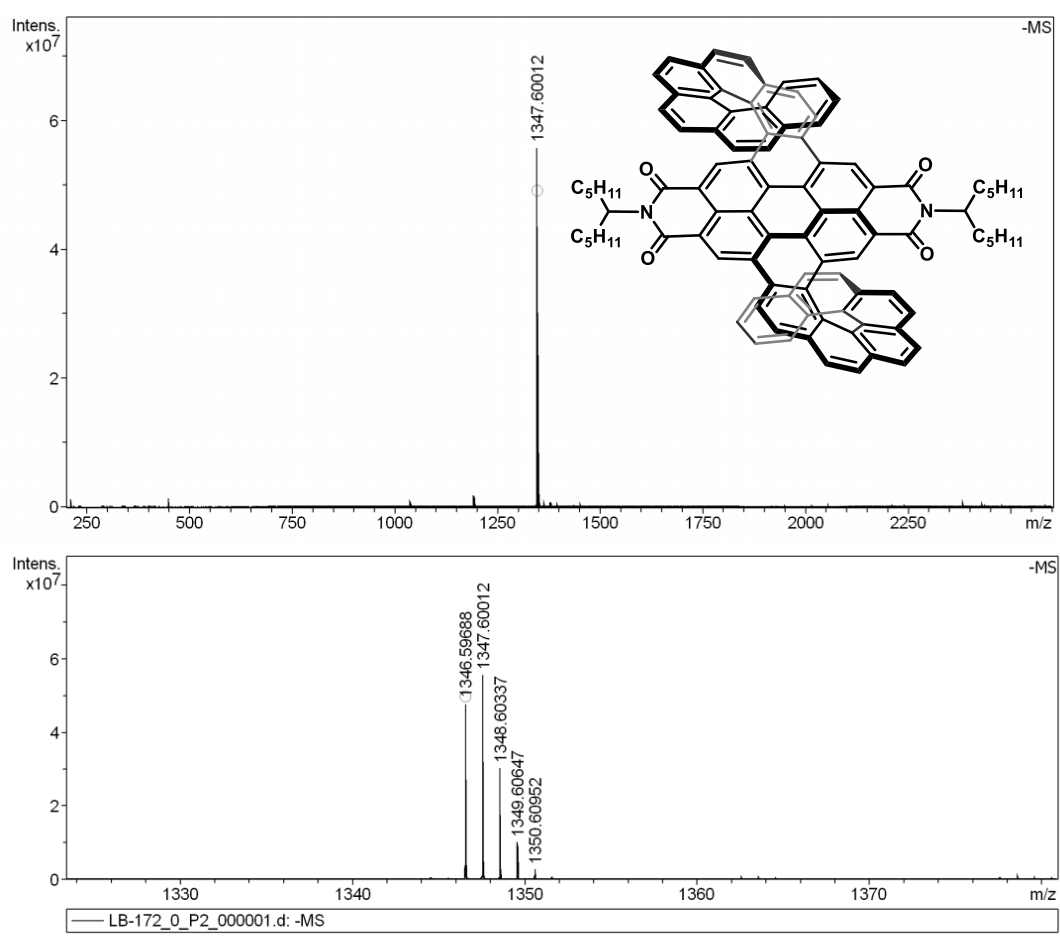

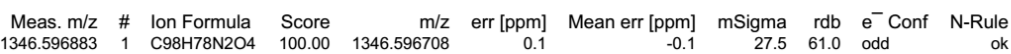

Figure S61. High-resolution mass spectra (HRMS) of compound PD8H-7M 


\section{References}

(1) Rajasingh, P.; Cohen, R.; Shirman, E.; Shimon, L. J. W.; Rybtchinski, B. J. Org. Chem. 2007, 72, 5973-5979.

(2) Ball, M.; Zhong, Y.; Fowler, B.; Zhang, B.; Li, P.; Etkin, G.; Paley, D. W.; Decatur, J.; Dalsania, A. K.; Li, H.; Xiao, S.; Ng, F.; Steigerwald, M. L.; Nuckolls, C. Macrocyclization in the Design of Organic n-Type Electronic Materials. J. Am. Chem. Soc. 2016, 138, 12861-12867.

(3) Jakubec, M.; Beránek, T.; Jakubík, P.; Sýkora, J.; Žádný, J.; Církva, V.; Storch, J. 2-Bromo[6]helicene as a Key Intermediate for [6]Helicene Functionalization. J. Org. Chem. 2018, 83, 3607-3616.

(4) M. J. Frisch, G. W. Trucks, H. B. Schlegel, G. E. Scuseria, M. A. Robb, J. R. Cheeseman,G. Scalmani, V. Barone, B. Mennucci, G. A. Petersson, H. Nakatsuji, M. Caricato, X. Li,H. P. Hratchian, A. F. Izmaylov, J. Bloino, G. Zheng, J. L. Sonnenberg, M. Hada, M. Ehara, K. Toyota, R. Fukuda, J. Hasegawa, M. Ishida, T. Nakajima, Y. Honda, O. Kitao, H. Nakai, T. Vreven, Jr. Montgomery, J. A., J. E. Peralta, F. Ogliaro, M. Bearpark, J. J. Heyd, E. Brothers, K. N. Kudin, V. N. Staroverov, R. Kobayashi, J. Normand, K. Raghavachari, A. Rendell, J. C. Burant, S. S. Iyengar, J. Tomasi, M. Cossi, N. Rega, M. J. Millam, M. Klene, J. E. Knox, J. B. Cross, V. Bakken, C. Adamo, J. Jaramillo, R. Gomperts, R. E. Stratmann, O. Yazyev, A. J. Austin, R. Cammi, C. Pomelli, J. W. Ochterski, R. L. Martin, K. Morokuma, V. G. Zakrzewski, G. A. Voth, P. Salvador, J. J. Dannenberg, S. Dapprich, A. D. Daniels, O. Farkas, J. B. Foresman, J. V. Ortiz, J. Cioslowski, and D. Fox, 2009. Gaussian 09, Revision D.01, Gaussian, Inc., Wallingford, CT.

(5) Becke, A. D. Density-functional thermochemistry. III. The role of exact exchange $J$. Chem. Phys. 1993, 98, 5648-5652; Stevens, P. J.; Devlin, F. J.; Chabalowski, C. F.; Frisch, M. J. J. Phys. Chem. 1994, 98, 11623-11631.

(6) Tomasi, J.; Mennucci, B.; Cammi. R. Quantum Mechanical Continuum Solvation Models. Chem. Rev. 2005, 105, 2999-3093. 
(7) Helgaker, T.; Jørgensen, P. An electronic Hamiltonian for origin independent calculations of magnetic properties. J. Chem. Phys. 1991, 95, 2595-2601. 